\title{
O teorema de Bishop-Phelps \\ e \\ alguns resultados associados
}

\author{
Fábio Skilnik
}

Dissertação Apresentada ao INSTITUTO DE MATEMÁTICA E ESTATÍSTICA da UNIVERSIDADE DE SÃO PAULO para a obtenção do grau de mestre em matemática

Área de concentração: Análise

Orientadora: Profa. Dra. Mary Lilian Lourenço

São Paulo, outubro de 1998. 


\title{
O teorema de Bishop-Phelps e alguns resultados associados
}

\author{
Este exemplar corresponde à redaçào final \\ da dissertação devidamente corrigida e \\ defendida por Fábio Skilnik \\ e aprovada pela comissão julgadora.
}

Sào Paulo, novembro de 1998.

Banca examinadora:

- Profa. Dra. Mary Lilian Lourenço (Orientadora) : IME - USP

- Prof. Dr. Jorge Tulio Mujica Ascui : IMECC - UNICAMP

- Prof. Dra. Luiza Amália de Moraes : UFRJ 
"Ando devagar, porque já tive pressa e levo esse sorriso, porque já chorei demais

Hoje me sinto mais forte, mais feliz, quem sabe

Eu só levo a certeza, de que muito pouco eu sei, ou nada sei ..."

(trecho de Tocando em frente, Almir Sater/Renato Teixeira)

Aos queridos $\varepsilon$ e $\delta$, bem como aos meus heróis: Mahatma Gandhi, Richard Feynman e Winston Smith.

Em termos mais concretos, gostaria de homenagear:

Meu irmão Cláudio Skilnik, por quem qualquer admiração é pequena.

Minha avó Gisela Skilnik (in memoriam) que, tenho certeza, entende e apóia seu neto que apaixonou-se irremediavelmente pela Matemática e meus avós Ada e Júlio Krygier, responsáveis por muitos de meus melhores momentos da infância.

A amiga e professora Mary Lilian Lourenço. Esta dissertação é fruto de seus esforços na difícil tarefa de me ensinar matemática (e também, claro, de sua ajuda nas correções, observações, sugestões e muitas revisões). 


\section{Agradecimentos}

Aos sempre amigos e mestres Carmen S. Cardassi e Alfredo J. Aragona, com gratidão pelos extraordinários anos de iniciação científica com a primeira e o maravilhoso curso de "Funções de várias variáveis complexas" no qual tive o grande prazer de conhecer o segundo.

Aos vários professores do IME que, desde minha graduação, têm estado presentes me ensinando, motivando e dando oportunidades. Entre eles gostaria de ressaltar: Hamilton L. Guidorizzi, Jacques Bouchara, Roseli Fernandez, Élvia M. Sallum, Rosa M. Chaves, Vera Giusti, Augusto Reynol Filho, Leilá Figueiredo e Cláudio Gorodski.

Aos professores da comissão julgadora de minha dissertação. Suas muitas sugestões e correções contribuiram fortemente para as melhorias presentes nesta versào final.

Aos funcionários da biblioteca (Sílvia, Verinha, Max, Luiz, etc), secretaria de pós-graduação (Lilian, Franscisca e D.Ana) e xerox (Marlene's) pelo carinho, apoio e ajuda incomuns.

A talentosa amiga Marcela Irene Merklen (e família!), que decidiu acreditar no "meu estilo" desde o princípio (e apesar dos percalços).

Aos meus amigos e primos Dorinha e Tobias Skrzeczanovski, que sabem o que tudo isso significa para mim.

Aos meus queridos e admirados amigos João Alberto Domenici, Ricardo dos Reis Rosa e Pedro de Almeida Camargo, com quem tive o prazer de trabalhar na área de derivativos. 
As minhas queridas Adriana Damasceno da Silva, Clarice M. de Gouvêa (e Alfredo), Cristiane Pereira, Paula Lev, Márcia Regina Gallego e, claro, aos amigos Daniel Wahba e Norberto C.Carreira.

Ao meu amigo Maurício Machado Galvão, um exemplo de raro talento em matemática.

Aos demais estudantes do IME com quem tive o prazer de me relacionar, dentre os quais: Wânia Cristina de Lucca, Ana Lúcia Fernandes, Luciano O.C.Huanca, Jair Donadelli, Claus A. Matsushigue, Cibele Dunder, Caroline Bardini, José C.S. de Miranda, Eliane C. Yasawa, Paula Lev, Adriana Hissadomi, Juaci P. da Silva, Antonio Munhoz, Lúcia Ikemoto, Luciene G. Santos, Sandra Sartori, Sonia Huamani, Delhi e Betty Lopez.

A dois professores de minha graduação em Ergenharia: Euryale de Jesus Zerbini e Arlindo Tribess.

A alguns dos grandes professores que tive antes do terceiro grau: José Stockel Filho, Gita Ghinzburg, Álvaro Zimmermann Aranha, Manoel Benedito Rodrigues, Bartolomeo Albanese, José Carlos Estevinho Lopes, Amadeu Matsumura, Gil Marcos Ferreira, Rabino Yehuda Busquila.

Aos meus bons amigos originários do Banco Tendência: Henry, Malta, Luciana, Gil, Dudu, Fabiana, Kênia, Maria Alice, Tobaruella e Ike, que com simpatia me permitiu conhecer o mundo financeiro.

A Sra. Euvarita Aparecida da Silva ("Cida"), que tem me ajudado muito.

Ao $\mathrm{CNPq}$, pelo apoio financeiro.

Por último ("but not least"), aos meus alunos, ainda que meu esforço sincero seja incomparável ao talento daqueles com quem tive o prazer de aprender.

"The mathematician's patterns, like the painter's or the poet's must be beautiful; the ideas, like the colours or the words, must fit together in a harmonious way. Beauty is the first test ; there is no permanent place in the world for ugly mathematics."

(G.H. Hardy) 


\section{Resumo}

Este trabalho tem por objetivo estudar o teorema clássico de BishopPhelps e os primeiros passos dados no sentido de sua generalização natural: para quais espaços de Banach $X, Y$ o conjunto dos operadores lineares contínuos de $X^{r}$ em $Y$ que atingem suas respectivas normas é denso no conjunto dos operadores lineares limitados definidos nestes mesmos espaços? Apresentamos aqui vários resultados de J.Lindenstrauss ([Lindenst]): é sempre verdade quando $X$ for reflexivo mas, em geral, a resposta é negativa (ainda que $Y=X$ ). Restringiremos nosso estudo aos fatos por ele obtidos relacionados com a "propriedade $A$ "; a convexidade das bolas unitárias e outras características geométricas dos espaços de Banach envolvidos terão interessantes consequências. Quanto à "propriedade $B$ ", definimos o espaço estudado por W.T.Gowers ([Gowers]) e apresentamos sua prova de que os espaços $l^{p}$ 's $(1<p<\infty)$ não a possuem. Explorando características do espaço de Gowers encerramos o trabalho com um exemplo de D.Acosta, F.Aguirre e R.Payá ([A.A.P.2]) que mostra a não existência, para um espaço de Banach qualquer, de um teorema de Bishop-Phelps no contexto das formas bilineares.

\section{Abstract}

The main purpose of this work is to study the classical Bishop-Phelps theorem and the first steps made to its natural generalization: to which pairs of Banach spaces $X, Y$ is it true that the set of norm-attaining linear operators from $X$ to $Y$ is dense in the set of bounded linear operators from $X$ to $Y$ ? We shall present here several results by Lindenstrauss ([Lindenst]): it is always the case if $X$ is reflexive and, in general, the answer is negative (even when $Y=X$ ). We shall restrict ourselves to his results related to "property $A$ "; the rule played by the unit cells' convexity and other geometric features of the Banach spaces concerned do have far-reaching consequences. As far as Lindenstrauss' "property $B$ " is concerned, we concentrate on the Banach space used by W.T.Gowers ([Gowers]). We present his proof of the fact that $l^{p}(1<p<\infty)$ does not have "property $B$ " and, getting the most out of Gowers space properties, we shall exhibit an example due to D.Acosta, F.Aguirre and R.Payá ([A.A.P.2]) on the (non-existence of a) bilinear version of the Bishop-Phelps theorem on an arbitrary Banach space. 


\section{Introdução}

A clássica caracterização dos espaços reflexivos em espaços de Banach (devida à R.C.James) dada pelos espaços cujos funcionais lineares contínuos atingem suas respectivas normas apresenta uma questão bastante natural: os funcionais que não têm tal propriedades sào "aproximáveis" pelos que a possuem ou, de outra forma, há densidade do conjunto formado pelos que atingem suas normas no conjunto dos lineares limitados? - E.Bishop e R.R.Phelps respondem afirmativamente (para todo espaço de Banach) no artigo publicado em 1961, dando origem às questões referentes às possíveis generalizações do resultado.

J.Lindenstrauss pioneiramente estuda sistematicamente o problema e, em 1963, apresenta um artigo que viria a orientar fortemente a pesquisa nesta área nos anos posteriores. Acreditando que a questão envolvida entre operadores lineares limitados definidos e tomando valores em quaisquer espaços de Banach é demasiadamente geral, define os espaços possuidores das propriedades $A$ e $B$. A geometria de espaços de Banach ocupa papel central nos resultados por ele obtidos e, nesta publicação, ganham destaque algumas consequências da existência ou não de tais propriedades nos espaços estritamente ou localmente uniformemente convexos.

Em 1990 W.T.Gowers apresenta um espaço de Banach que acaba por resolver algumas questões até então em aberto. Valendo-se de argumentos relacionados com a compacidade de determinados operadores, ele é capaz de garantir a não existência da propriedade $B$ para vários espaços, em particular para os $l^{p}$ 's $(1<p<\infty)$. Além disso, D.Acosta, F.Aguirre e R.Payá apresentam em 1996 um (contra-)exemplo que garante a não existência, para um espaço de Banach qualquer, de um teorema do "tipo Bishop-Phelps" no contexto das formas bilineares contínuas, no qual o espaço de Gowers é fundamental.

Este trabalho tem por objetivo estudar os resultados acima mencionados, que podem ser encontrados nas seguintes referências: [Holmes], [Lindenst], [Gowers], [A.A.P.1] e [A.A.P.2]. Salientamos, porém, que muitas técnicas e propriedades associadas ao tema não foram aqui abordadas, dentre as quais mencionamos: a equivalência demonstrada por J.Bourgain entre as propriedades de Bishop-Phelps e Radon-Nikodym ([Bourgain]), o princípio de "oti- 
mização" de C.Stegall ([Stegall]) que permitiu a R.Aron, C.Finet e E.Werner mostrarem a existência do teorema de Bishop-Phelps para formas n-lineares nos espaços de Banach que têm a propriedade de Radon-Nikodym ([A.F.W.]) ou ainda resultados de renormagem como os obtidos por J.R.Partington que garantem, por exemplo, o fato de todo espaço de Banach ser isomorfo a um espaço cujos operadores norm-attaining constituem subconjunto denso nos lineares limitados ([Partington]).

A seguir descrevemos de maneira sucinta o conteúdo dos diversos capítulos.

No capítulo 1 apresentamos algumas notações, definições e resultados utilizados nos demais. No seguinte, apresentamos os cones, pontos-suporte cônicos e resultados como o teorema de Phelps-Bronsted-Rockafellar que nos permitem provar os chamados "teoremas de Bishop-Phelps", incluindo o "clássico" mencionado no início desta introdução ([Holmes]).

No capítulo 3 apresentamos a quase totalidade dos resultados associados à propriedade $A$ publicados no artigo de J.Lindenstrauss ([Lindenst]) que, para tanto, necessitam de diversas definições e propriedades associadas à estrita ou local uniformidade convexa de (normas em) espaços de Banach, bem como dos conceitos de pontos extremais/expostos. No último capítulo definimos o espaço de Gowers ([Gowers]) e apresentamos várias propriedades dele. Algumas delas, obtidas por D.Acosta, F.Aguirre e R.Payá ([A.A.P.1] e [A.A.P.2]) possibilitaram a descoberta (pelos mesmos) de um contra-exemplo no contexto das formas bilineares contínuas. 


\section{Sumário}

1 Definições e resultados preliminares 5

1.1 Notação . . . . . . . . . . . . . . . . . . . . 5

1.2 Definições e resultados . . . . . . . . . . . . 6

2 O teorema de Bishop-Phelps 17

2.1 Introdução . . . . . . . . . . . . . . . 17

2.2 Espaços vetoriais pré-ordenados e cones . . . . . . . . . . 18

2.3 Os teoremas de Bishop-Phelps . . . . . . . . . . . . 25

3 Alguns resultados sobre operadores entre espaços de Banach que atingem suas normas $\quad 32$

3.1 Introdução . . . . . . . . . . . . . . . . 32

3.2 Primeiros resultados . . . . . . . . . . . . . . 33

3.3 Espaços estritamente e uniformemente convexos . . . . . . . . 46

3.4 Pontos extremais, expostos e a propriedade $A \ldots \ldots \ldots$

4 O espaço de Gowers e um contra-exemplo do Bishop-Phelps para bilineares 83

4.1 Introdução . . . . . . . . . . . . . . . . . . . 83

4.2 O espaço de Gowers e algumas aplicaçòes . . . . . . . . . . 84

4.3 Bishop-Phelps para bilineares . . . . . . . . . . . . . . 94

$\begin{array}{ll}\text { Referências bibliográficas } & 99\end{array}$ 


\section{Capítulo 1}

\section{Definições e resultados preliminares}

Apresentamos neste capítulo as notações, definições e resultados necessários no decorrer deste trabalho e cujas demonstrações podem ser encontradas nas respectivas referências. Todos os espaços vetoriais tratados nesta dissertaçâo serão considerados sobre o corpo dos números reais.

\subsection{Notação}

Considere fixada a nomenclatura que se segue.

IN o conjunto dos números inteiros estritamente positivos

$X$ espaço vetorial (normado/Banach)

0 a origem do espaço vetorial em questão (o vetor nulo)

$B_{X}(\|\cdot\|)$ a bola unitária, dada por $\left\{x \in X^{\prime}:\|x\| \leq 1\right\}$

$\mathcal{S}_{X}(\|\cdot\|)$ a esfera unitária, dada por $\left\{x \in X^{\prime}:\|x\|=1\right\}$

$\operatorname{int}(A)$ o interior de um subconjunto $A$ de $X$

$\mathcal{L}\left(X_{1}, X_{2}\right)$ o espaço das aplicações lineares e contínuas de $X_{1}$ em $X_{2}$

$X^{\prime}, X^{\prime \prime}$ o dual $(\mathcal{L}(X, \mathbb{R}))$ e bidual $\left(\left(X^{\prime}\right)^{\prime}\right)$ topológicos do espaço $X^{\prime}$

$\left(x_{i}\right)_{i \geq 1} \subset A$ sequência cujos elementos pertencem ao subconjunto $A$ de $X^{r}$

A menos de menção contrária, as propriedades topológicas envolvidas no decorrer da dissertação estarão associadas à topologia dada pela norma em questão. Teremos, por exemplo, o interior, o fecho, a densidade ou a convergência assim entendidos. 
Ao escrever " $N \geq 1$ " admite-se que $N$ seja inteiro nestas condições; entenderemos $\gamma$ dado em " $0<\gamma<1$ " ou em " $\gamma \in(0,1)$ " como sendo um número real. Além disso, utilizaremos algumas vezes a notaçào " $[x, y]$ " para o segmento de reta que une os vetores $x$ e $y$ (o contexto deixará isso claro).

Intencionando facilitar a leitura, utilizaremos os seguintes símbolos:

- indicando o final de uma demonstração

* indicando o final de uma afirmação (em exemplos, provas,etc.)

\subsection{Definições e resultados}

\section{Definição 1.1 (conjunto simétrico)}

Um subconjunto $\Omega$ de um espaço vetorial $X$ é dito simétrico (em relação à origem) se $x \in \Omega \Rightarrow-x \in \Omega$.

\section{Definição 1.2 (envoltória convexa)}

Considere $A$ um subconjunto não vazio do espaço vetorial X. A interseçấo de todos conjuntos convexos de $\mathrm{X}$ que contém A constitui um conjunto convexo de $X$, denominado de envoltória convexa de $A$. Ele será designado a partir daqui por conv $(A)$.

Considerando $X$ normado, a envoltória convexa fechada, designada por $\overline{\operatorname{conv}}(A)$, deve ser entendida como o fecho em $X$ da envoltória convexa $\operatorname{conv}(A)$ em questão.

\section{Teorema 1.3}

Seja A um subconjunto nâo vazio de um espaço vetorial. A envoltória convexa de $A$ coincide com o conjunto de todas combinaçôes convexas

$$
x=\sum_{i \in I} \alpha_{i} x_{i}
$$

onde $\left\{x_{i}\right\}_{i \in I} \subset A$ e $\left\{\alpha_{i}\right\}_{i \in I} \subset[0,1]$ é tal que $\sum_{i \in I} \alpha_{i}=1$, com $\alpha_{i}=0$ a menos de um número finito de índices.

Prova: Vide por exemplo [Berberian], Corol (25.14), 103. 


\section{Definição 1.4 (separabilidade)}

Um espaşo normado $(X,\|\cdot\|)$ é dito separável se admite um subconjunto enumerável denso (nele).

\section{Definição 1.5 (base de Schauder)}

Considere $(X,\|\cdot\|)$ um espaço normado. Se $X$ contém uma sequência $\left(e_{n}\right)_{n \geq 1}$ tal que, para cada $x \in X^{r}$ existe uma única sequência de escalares $\left(\lambda_{n}\right)_{n \geq 1}$ que satisfaz

$$
\left\|x-\sum_{i=1}^{n} \lambda_{i} \cdot \epsilon_{i}\right\| \stackrel{n \rightarrow \infty}{\longrightarrow} 0,
$$

dizemos que $\left(e_{n}\right)_{n \geq 1}$ constitui uma base de Schauder de $X$.

\section{Definição 1.6 (aplicações lineares limitadas)}

Sejam $(X,\|\cdot\|)$ e $(Y,\|\cdot\|)$ dois espaços normados. Um operador linear $T: X \rightarrow Y$ é dito limitado se existe uma constante $k \geq 0$ tal que $\|T x\| \leq k \cdot\|x\|$, para todo $x \in X$.

\section{Teorema 1.7}

Sejam $(X,\|\cdot\|)$ e $(Y,\|\cdot\|)$ dois espaços normados. Um operador linear $T: X \rightarrow Y$ é contínuo se e só se é limitado.

Prova: Vide por exemplo [KitrAklv], Teor 1, 128.

\section{Teorema $1.8(\mathcal{L}(X, Y)$ é Banach $)$}

Sejam $(X,\|\cdot\|)$ e $(Y,\|\cdot\|)$, respectivamente, espaços normado e Banach e $\mathcal{L}(X, Y)$ o espaço formado pelos operadores lineares e contínuos de $X \mathrm{em}$ $Y$. A aplicação $\|\cdot\|: \mathcal{L}(X, Y) \rightarrow \mathbb{R}$ definida para cada $T \in \mathcal{L}(X, Y)$ por

$$
\|T\|=\sup _{x \in \mathcal{B}_{X}(\|\cdot \cdot\|)}\|T(x)\|\left(=\sup _{x \in \mathcal{S}_{X}(\|\cdot\|)}\|T(x)\|\right)
$$

constitui uma norma em $\mathcal{L}(X, Y)$ (a que usaremos a menos de mençâo contrária) que o faz completo.

Prova: Vide por exemplo [Berberian], Teor (40.7), 168. 


\section{Teorema 1.9}

Todo subespaço fechado de um espaço de Banach $(\mathrm{X},\|\cdot\|)$ é um espaço de Banach.

Prova: Vide por exemplo [Berberian], Teor (16.11), 67.

\section{Teorema 1.10 (fechados encaixantes)}

Sejam X espaço de Banach $\in\left(F_{n}\right)_{n \geq 1}$ uma sequência de conjuntos não vazios, fechados $\epsilon m X$ e tais que $F_{1} \supset F_{2} \supset F_{3} \supset \ldots \operatorname{com} \operatorname{diam}\left(F_{n}\right) \stackrel{n \rightarrow \infty}{\longrightarrow} 0$. Então existe um ponto $x_{0} \in X$ tal que $\bigcap_{n \geq 1} F_{n}=\left\{x_{0}\right\}$.

Prova: Veja por exemplo [BrwnPrcy], Pg 180.

\section{Definição 1.11 (conjunto sólido)}

Considere $(X,\|\cdot\|)$ um espaço normado. Um conjunto A nele contido é dito sólido se seu interior é não vazio.

\section{Teorema 1.12 (propriedades frente à convexidade)}

Seja $(X,\|\cdot\|)$ um espaço normado. O fecho de todo subconjunto convexo A de $X$ também é convexo e, além disso, se A for sólido, seu interior também é convexo.

Prova: Vide [Berberian], Teor (25.17) e (25.20), 103 e 105.

Definição 1.13 (hiperplano(-suporte), separação de conjuntos)

Considere fixado um espaço normado $(X,\|\cdot\|)$.

- Dados $f \in \mathrm{X}^{\prime}, f \neq 0$ e a real. definiremos o hiperplano $\mathcal{H}(f, a)$ como sendo dado pelo conjunto

$$
\mathcal{H}(f, \alpha)=\{x \in X: f(x)=\alpha\} .
$$

- Considere o hiperplano $\mathcal{H}(f, \alpha)$ e os semi-espaços por ele determinados

$$
\{x \in X: f(x) \leq \alpha\},\{x \in X: f(x) \geq \alpha\} \text {. }
$$

Dizemos que dois subconjuntos não vazios $A, B$ de $X$ podem ser separados (pelo hiperplano $\mathcal{H}(f, \alpha)$ ) se estâo contidos em semi-espaços 
distintos determinados por algum hiperplano $(\mathcal{H}(f . a))$. Em outras palavras, se existe um funcional nâo nulo $f \in X^{\prime} \in$ a real tais que

$$
\sup _{x \in A} f(x) \leq \alpha \leq \inf _{x \in B} f(x) .
$$

Se uma das desigualdades acima puder ser estrita (portanto ambas), dizemos que $A$ e $B$ podem ser estritamente separados (pelo hiperplano $\mathcal{H}(f, \alpha))$.

- Dizemos que $\mathcal{H}(f, \alpha)$ é um hiperplano-suporte em $\xi \in A$ para o subconjunto $A$ de $X$ se $\alpha \in \mathbb{R}$ é tal que $f(\xi)=\alpha$ e A está contido em $\{x \in X: f(x) \leq \alpha\}$ ou em $\left\{x \in X^{\prime}: f(x) \geq \alpha\right\}$.

É importante notar que os papéis de $A$ e $B$ nas desigualdades dadas acima são "cambiáveis", bastando tomar $-f$ no lugar da $f$ considerada.

\section{Teorema 1.14 (teorema da separação)}

Sejam $A$ e $B$ subconjuntos não vazios e convexos de um espaço normado $X$. Se $A$ é um sólido e $\operatorname{int}(A) \cap B=\emptyset$, então $A \in B$ podem ser separados. Além disso, se $A$ e $B$ forem abertos em $X$, podem ser estritamente separados.

Prova: Vide por exemplo [KtrAklv], Teor 5, 72.

\section{Corolário 1.15 (teorema do suporte)}

Um subconjunto $A$ sólido, convexo e fechado num tspaço normado $X$ admite em cada um de seus pontos de fronteira um hiperplano-suporte.

Prova: Basta observar que $A$ contém todos os seus pontos de fronteira; dado qualquer um deles em particular, digamos $\xi$, tem-se $\operatorname{int}(A) \cap\{\xi\}=\emptyset$ e o teorema anterior se aplica.

\section{Teorema 1.16 (forma geométrica do Hahn-Banach)}

Sejam $A$ e $B$ subconjuntos não vazios, convexos e disjuntos de um espaço normado $X$. Caso $A$ seja fechado e $B$ compacto, $A$ e $B$ podem ser estritamente separados.

Prova: Veja por exemplo [Brezis], Teor (1.7), 7. 


\section{Teorema 1.17}

Para cada elemento não nulo $x_{0}$ de um espaço normado $\left(X^{\prime},\|\cdot\|\right)$. existe um funcional $f \in \mathcal{S}_{X^{\prime}}(\|\cdot\|)$ tal que $f\left(x_{0}\right)=\left\|x_{0}\right\|$.

Prova: Vide por exemplo [KtrAklv], Teor 2, 176.

\section{Teorema 1.18}

Todo funcional linear contínuo definido num subespaço de um espaço normado $(X,\|\cdot\|)$ pode ser estendido para todo $X$ de forma a preservar sua norma.

Prova: Vide por exemplo [Maddox], Cap 5, Teor 6, Corol 1, 134.

\section{Teorema 1.19}

Para cada elemento $x$ pertencente ao espaço normado $(X,\|\cdot\|)$ temos

$$
\|x\|=\sup _{f \in \mathcal{B}_{x^{\prime}}(\|\cdot \cdot\|)}|f(x)| \text {. }
$$

Prova: Vide por exemplo [KitrAklv], 176.

\section{Teorema 1.20}

Seja $W$ um subespaço fechado de um espaço normado $(X,\|\cdot\|)$. Então $W$ é compacto se e só se toda sequência em W admite uma subsequência convergente.

Prova: Vide por exemplo [Maddox], Teor 21, 62.

\section{Teorema 1.21}

Seja $(X,\|\cdot\|)$ um espaço normado. Entâo $X$ tem dimensâo finita se $e$ somente se todo subconjunto limitado é relativamente compacto.

Prova: Vide por exemplo [KtrAklv], 88. 


\section{Teorema 1.22 (aplicação aberta)}

Sejam $X, Y$ espaços de Banach $\epsilon T \in \mathcal{L}(X, Y)$ sobrejetiva. Então $T$ é uma aplicação aberta.

Como consequência, caso $T$ seja bijetiva, linear e contínua será então um isomorfismo (i.e, um homeomorfismo linear).

Prova: Vide por exemplo [Berberian] Teor (48.1), 202.

\section{Definição 1.23 (aplicações compactas)}

Considere $X, Y$ dois espaços normados. Uma aplicação $T \in \mathcal{L}(X, Y)$ é dita compacta se dado qualquer $A \subset X$ limitado, $T(A)$ é subconjunto relativamente compacto de $Y$.

\section{Teorema $\mathbf{1 . 2 4}$}

Sejam $(X,\|\cdot\|)$ e $(Y,\|\cdot\|)$ espaços normado $\epsilon$ Banach, respectivamente. Se a sequência de operadores compactos $\left(T_{n}\right)_{n \geq 1} \subset \mathcal{L}(X, Y)$ converge para um operador $T(T \in \mathcal{L}(X, Y))$, entâo $T$ também é compacto.

Prova: Vide por exemplo [KitrAklv], 246.

\section{Definição 1.25 (aplicações adjunta e bi-adjunta)}

Sejam $(X,\|\cdot\|)$ e $(Y,\|\cdot\|)$ dois espaços normados e $T \in \mathcal{L}(X, Y)$.

Definimos a aplicação adjunta $T^{\prime}: Y^{\prime} \rightarrow X^{\prime}$ associada a $T$ como sendo dada, em cada $f \in Y^{\prime}$ por

$$
\left(T^{\prime} f\right)(x)=f(T x) \forall x \in X .
$$

Note que $T^{\prime} f \in X^{\prime}$ e que $T^{\prime}: Y^{\prime} \rightarrow X^{\prime}$ assim definida é linear e limitada (teorema (1.28)).

Podemos da mesma forma definir a aplicação bi-adjunta $T^{\prime \prime}=\left(T^{\prime}\right)^{\prime}$ associada a $T$ como sendo dada, em cada $g \in X^{\prime \prime}$ por

$$
\left(T^{\prime \prime} g\right)(f)=g\left(T^{\prime} f\right) \forall f \in Y^{\prime} .
$$

Aqui também temos $T^{\prime \prime} g \in Y^{\prime \prime} \in T^{\prime \prime}: X^{\prime \prime} \rightarrow Y^{\prime \prime}$ linear e limitada (teorema (1.28)). 


\section{Definição 1.26 (imersão canônica)}

Considere $(X,\|\cdot\|)$ um espaço normado. A imersão canônica de $X$ no seu bidual $X^{\prime \prime}$ é definida como sendo a aplicaçào $J: X^{\prime} \rightarrow X^{\prime \prime}$ dada por $x \in X \mapsto J(x) \in X^{\prime \prime}$ onde $J(x)$ é definido por

$$
J(x)(f)=f(x), \forall f \in X^{\prime} .
$$

\section{Teorema 1.27}

Considere $(X,\|\cdot\|)$ um espaço normado. A imersâa canônica $J: X \rightarrow X^{\prime \prime}$ é uma isometria linear contínua de $\mathrm{X}$ em $\mathrm{X}^{\prime \prime}$.

Prova: Vide por exemplo [Berberian], Teor (40.13), 169.

Teorema 1.28 (propriedades da adjunta e bi)

Sejam $(X,\|\cdot\|)$ e $(Y,\|\cdot\|)$ espaços normados. $\lambda \in \mathbb{R}, S, T \in \mathcal{L}(X, Y)$ e $J$ a imersảo canônica do espaço em questão. Então

- $(S+T)^{\prime}=S^{\prime}+T^{\prime}$,

- $(\lambda T)^{\prime}=\lambda T^{\prime}$,

- $T^{\prime \prime}(J(x))=J(T(x)), \forall x \in X$,

- $\left\|T^{\prime}\right\|=\|T\|$,

- $T^{\prime}$ será uma isometria sobrejetiva sempre que $T$ o for.

Prova: Veja por exemplo [Berberian], Teor (40.19), Pag. 171.

\section{Definição 1.29 (topologias fraca e fraca-estrela)}

Seja $(X,\|\cdot\|)$ um espaço normado.

- Definimos em $X^{\prime}$ a topologia fraca $\sigma\left(X^{\prime}, X^{\prime}\right)$ como sendo a topologia obtida tomando-se como base de vizinhanças de cada ponto $\xi \in X$ os conjuntos da forma

$$
V_{\xi}=\left\{x \in X: \sup _{i \in I}\left|f_{i}(x-\xi)\right|<\varepsilon\right\}
$$

com $I \subset\{1,2, \ldots\}$ finito. $\left\{f_{i}\right\}_{i \in I} \subset X^{\prime} \in \varepsilon>0$. 
- Definimos em $\mathrm{X}^{\prime}$ a topologia fraca-estrela $\sigma\left(\mathrm{X}^{\prime}, \mathrm{X}^{\prime}\right)$ como sendo a topologia obtida tomando-se como base de vizinhanças de cada funcional $\varphi \in X^{\prime \prime}$ os conjuntos da forma

$$
V_{\varphi}=\left\{f \in X^{\prime}: \sup _{i \in I}\left|(f-\varphi)\left(x_{i}\right)\right|<\varepsilon\right\}
$$

com $I \subset\{1,2, \ldots\}$ finito, $\left\{x_{i}\right\}_{i \in I} \subset X \in \varepsilon>0$.

\section{Teorema 1.30 (Banach-Alaoglu-Bourbaki)}

Sendo $\left(X^{\prime},\|\cdot\|\right)$ um espaço de Banach, a bola unitária $\mathcal{B}_{X^{\prime}}(\|\cdot\|) \quad$ é fraca-estrela compacta em $X^{\prime \prime}$.

Prova: Vide por exemplo [Brezis], 42.

\section{Teorema 1.31}

Seja $(X,\|\cdot\|)$ um espaço de Banach. A bola unitária $\mathcal{B}_{X^{\prime}}(\|\cdot\|)$ é fracaestrela metriável em $X^{\prime \prime}$ se e só se $X$ é separável.

Prova: Vide por exemplo [Dunf.Schw.], Teor 2, V.5.1, 426.

\section{Teorema 1.32 (Goldstine)}

Sejam $X$ um espaço de Banach e $J: X \rightarrow X^{\prime \prime}$ a imersão canônica de $X$ em $X^{\prime \prime}$. Então a imagem $J\left(\mathcal{B}_{X}(\|\cdot\|)\right)$ é fraca-estrela densa na bola unitária $\mathcal{B}_{X^{\prime \prime}}(\|\cdot\|)$.

Prova: Vide por exemplo [Brezis], 44.

\section{Definição 1.33 (os espaços $L^{p}{ }^{\prime} s$ )}

Dado qualquer $1 \leq p<\infty$, considere $L^{p}([0,1])$ como sendo o conjunto das classes de equivalência ("iguais quase-sempre") das funções $x=x(t)$ definidas em $[0,1]$, tomando valores reais, Lebesgue-mensuráveis e tais que $|x(t)|^{p}$ seja Lebesgue-integrável em $[0,1]$.

As operações usuais de adição e multiplicação por escalar fazem de $L^{p}([0,1])$ um espaço vetorial e a aplicaçâo definida no espaço a valores em $\mathbb{R}$ dada por

$$
\|x\|=\left(\int_{0}^{1}|x(t)|^{p} \mu(d t)\right)^{1 / p}
$$

( $\mu$ medida de Lebesgue) constitui uma norma (a "usual") em $L^{p}([0,1])$ que o faz completo (veja [KtrAklv], 100 e 101). 
Como caso particular dos espaços $L^{p}$ 's (valendo-nos da medida de contagem) podemos definir os chamados espaços $l^{p}{ }^{\prime} s$. É o que faremos a seguir.

Definição 1.34 (os espaços $l^{p}{ }^{\prime} s$ e $c_{0}$ )

- Dado qualquer $1 \leq p<\infty$, considere $l^{p}$ como sendo o conjunto das sequências de números reais $x=\left(x_{k}\right)_{k \geq 1}$ tais que a série $\sum_{k \geq 1}\left|x_{k}\right|^{p}$ converge.

As operaçôes usuais de adiçâo e multiplicação por escalar fazem de $l^{p}$ um espaço vetorial e a aplicação definida no espaço a valores em $\mathbb{R}$ dada por

$$
\|x\|_{p}=\left(\sum_{k \geq 1}\left|x_{k}\right|^{p}\right)^{1 / p}
$$

constitui uma norma em lp (a "usual") que o faz completo.

- Considere $l^{\infty}$ como sendo o conjunto das sequências de números reais $x=\left(x_{k}\right)_{k \geq 1}$ limitadas.

As operações usuais de adição e multiplicação por escalar fazem de $1^{\infty}$ um espaço vetorial e a aplicação definida no espaço a valores em $\mathbb{R}$ dada por

$$
\|x\|=\sup _{k \geq 1}\left|x_{k}\right|
$$

constitui uma norma em $l^{\infty}$ que o fa $z$ completo.

- Considerando as normas "usuais" definidas, o espaço de Banach lp é separável (para todo $1 \leq p<\infty$ ), porém $l^{\infty}$ não.

- Definimos c como sendo o subespaço de $l^{\infty}$ constituido pelas sequências de números reais $x=\left(x_{k}\right)_{k \geq 1}$ que convergem para zero. $\mathrm{Mu}$ nindo $c_{0}$ da norma induzida de $l^{\infty}$, o espaço normado obtido é Banach e, além disso, separável.

Para as provas dos resultados envolvidos veja [KitrAklv], 100 e 102. 


\section{Teorema 1.35 (Riesz)}

Dado qualquer $1<p<\infty$, considere o expoente conjugado q definido por $1 / p+1 / q=1$. Todo elemento $y=\left(y_{1}, y_{2}, \ldots\right) \in l^{q}$ define um funcional linear contínuo $\hat{y}: l^{p} \rightarrow \mathbb{R}$ através da expressâo

$$
\tilde{y}(x)=\sum_{k \geq 1} y_{k} \cdot x_{k} \quad \text { onde } x=\left(x_{k}\right)_{k \geq 1} \in l^{p} .
$$

Além disso, a aplicação $y \mapsto \tilde{y}$ constitui um isomorfismo isométrico de $l^{q}$ sobre o espaço dual de $l^{p}$.

Prova: Vide por exemplo [Edwards], Teor(4.2.1), 176.

\section{Definição 1.36 (o espaço das sequências de Lorentz)}

Considere dados $1 \leq p<\infty \in w=\left(w_{n}\right)_{n \geq 1}$ uma sequência não crescente de reais nâo negativos e tais que $w_{1}=1, \lim _{n \rightarrow \infty} w_{n}=0 \quad \in \quad \sum_{n \geq 1} w_{n}=\infty$.

Ao espaço vetorial ${ }^{1}$ constituido pelas sequências de números reais $x=\left(x_{n}\right)_{n \geq 1}$ tais que $\sup _{\pi \in \sigma}\left(\sum_{n \geq 1}\left|x_{\pi(n)}\right|^{p} \cdot w_{n}\right)^{1 / p}<\infty$, onde $\sigma$ é $o$ conjunto das permutações em $\mathbb{N}$, pode-se associar uma norma dada por

$$
\|x\|=\sup _{\pi \in \sigma}\left(\sum_{n \geq 1}\left|x_{\pi(n)}\right|^{p} \cdot w_{n}\right)^{1 / p}
$$

que o faz completo. O espaço de Banach assim obtido é conhecido como o espaço das sequências de Lorentz d(w,p) ([Lind.Tzaf.]).

Apresentamos agora um teorema associado a tais espaços, cujo conteúdo (e notação) ficarão mais claros no decorrer do último capítulo.

${ }^{1}$ Valendo-se, nas sequências, das operações de adição e multiplicação por real usuais. 


\section{Teorema 1.37}

Seja $w=\left(w_{n}\right)_{n \geq 1}$ como na definiçâo (1.36) $\epsilon$, para cada $n \geq 1$, considere a soma parcial $H_{n}=\sum_{k=1}^{n} w_{k}$, bem como $J \subset \mathbb{N}$ qualquer subconjunto de cardinalidade $|J|=n$. O espaço das sequências de Lorentz $d(w .1)$ admite como pré-dual o espaço de Banach $\left(d_{*}(u, 1),\|\cdot\|\right)$ definido por

$$
d_{*}(w, 1)=\left\{x=\left(x_{i}\right)_{i \geq 1} \in c_{0}: \varphi_{n}(x) \stackrel{n \rightarrow \infty}{\longrightarrow} 0\right\}
$$

onde $\varphi_{n}(x)=\left(1 / H_{n}\right) \sup _{|J|=n} \sum_{j \in J}\left|x_{j}\right|$ e norma $\|\cdot\|: d_{*}(w, 1) \rightarrow \mathbb{R}$ dada por

$$
\|x\|=\sup _{n \in \mathbb{N}} \varphi_{n}(x), \forall x \in d_{*}(w, 1) .
$$

Além disso, a base de Schauder canônica de co é uma base de Schauder de $\left(d_{x}(w, 1),\|\cdot\|\right)$.

Prova: Veja [Garling] ou [S.P.] . 


\section{Capítulo 2}

\section{O teorema de Bishop-Phelps}

\subsection{Introdução}

É simples mostrar que num espaço de Banach reflexivo $(X,\|\cdot\|)$ todo funcional linear contínuo atinge sua norma.

De fato, tomando qualquer $f \in X^{\prime}$ (não nulo, do contrário é trivial) valendo-nos do teorema (1.17) podemos afirmar que existe $F \in \mathcal{S}_{X^{\prime \prime}}(\|\cdot\|)$ tal que $F(f)=\|f\|$. Da reflexidade de $X$ existe um elemento $x_{F} \in X$ associado à $F$ pela imersão canônica $J$ (aqui bijetiva) e assim sendo

$$
\|f\|=F(f)=J\left(x_{F}\right)(f)=f\left(x_{F}\right) .
$$

Sendo $J$ uma isometria, $\left\|x_{F}\right\|=\|F\|=1$. donde concluimos que $x_{F}$ é um ponto da esfera no qual $f$ atinge sua norma.

Em 1950 R.C.James ([James]) provou a recíproca para espaços de Banach que admitem uma base de Schauder e V.Klee ([Klee]) no mesmo ano o fez para qualquer Banach separável. Finalmente em 1963 o primeiro ([James2]) resolve a questão para um espaço de Banach qualquer (veja [Diestel], Teor 3, 12), caracterizando os espaços reflexivos nos espaços de Banach através do (agora clássico) teorema que se segue.

\section{Teorema 2.1 ("James' reflexivity theorem")}

Um espaço de Banach $(X,\|\cdot\|)$ é reflexivo se e só se todo elemento de seu dual $X^{\prime}$ atinge a respectiva norma. 
Assim sendo, sabemos que dado qualquer $(X,\|\cdot\|)$ espaço de Banach não reflexivo, existe $f \in X^{\prime \prime}$ que não atinge sua norma. Criou-se então naquela época uma definição e problema naturais: a subreflexividade e a caracterização dos espaços de Banach subreflexivos.

Um espaço de Banach $(X,\|\cdot\|)$ era dito subreflexivo se o conjunto dos funcionais lineares e limitados que atingem suas respectivas normas é denso em $X^{\prime \prime}$.

Formalmente, que condições um espaço de Banach $\left(X^{\prime},\|\cdot\|\right)$ deve satisfazer para que, dados quaisquer $\varepsilon>0$ e $f \in X^{\prime}$, existam $g \in X^{\prime}$ e $x_{0} \in \mathcal{S}_{X}(\|\cdot\|)$ tais que

$$
\left|g\left(x_{0}\right)\right|=\|g\| \quad \text { e } \quad\|f-g\|<\varepsilon ?
$$

O objetivo do presente capítulo é explorar tais conceitos e apresentar a resposta, obtida primeiramente em 1961 por E.Bishop e R.R.Phelps (no [B.P.]) e que ficou (a partir de então) conhecida como o "teorema de Bishop-Phelps" : Todo espaço de Banach é subreflexivo.

\subsection{Espaços vetoriais pré-ordenados e cones}

\section{Definição 2.2 (espaço vetorial pré-ordenado)}

Dizemos que um espaço vetorial $X$ está munido de uma relação de préordem $\leq$ quando são satisfeitas, para quaisquer $x, y, z \in X$, duas propriedades:

a) $x \leq x e$

b) $x \leq y, y \leq z \quad \Rightarrow \quad x \leq z$.

Tal relaçâo de pré-ordem $\leq$ é dita compativel com as operaçốes de adição e multiplicação por escalar definidas no espaço vetorial quando temos adicionalmente

c) $x \leq y \quad \Rightarrow x+v \leq y+v \quad, \forall v \in X \quad e$

d) $x \leq y \Rightarrow \lambda x \leq \lambda y, \forall \lambda \geq 0$.

$O$ espaço vetorial $X$ munido de uma relação de pré-ordem $\leq$ compatível com as operaçôes nele definidas é denominado um espaço vetorial pré-ordenado. 


\section{Exemplo 2.3}

Considere o espaço vetorial $\mathrm{X}^{\prime}$ constituído pelas funções $x=x(t)$ definidas num conjunto $\mathcal{T}$, tomando valores em $\mathbb{R}$ e com as operações usuais de adição e multiplicação por escalar.

Podemos dizer que existe uma relação de pré-ordem "natural" neste espaço vetorial, a saber: dados $x, y$ em $X$ escreveremos $x \leq y$ se (e só se) $x(t) \leq y(t), \forall t \in \mathcal{T}$.

Claramente são válidas para quaisquer $x, y, z \in X$ as propriedades:

- $x \leq x$,

- $x \leq y, y \leq z \Rightarrow x \leq z$

- $x \leq y \Rightarrow x+v \leq y+v, \forall v \in X \mathrm{e}$

- $x \leq y \Rightarrow \lambda x \leq \lambda y, \forall \lambda \geq 0$.

Portanto, $(X, \leq)$ assim definido constitui um espaço vetorial pré-ordenado.

\section{Observação 2.4}

Note que $x \leq y$ é equivalente à $y-x \geq 0$ e, portanto, a relação $\leq$ fica bem determinada ao especificarmos quem são os "elementos positivos" $z \geq \mathbf{0}$.

\section{Definição 2.5 (cone)}

Considere $X$ um espaço vetorial e $K \subset X, K \neq \emptyset$. Dizemos que $K$ é um cone em $X$ (com vértice na origem 0 ) se:

a) $x \in K \Rightarrow \lambda x \in K, \forall \lambda \geq 0$ e

b) $x, y \in K \Rightarrow x+y \in K$.

Costuma-se escrever tais propriedades que definem um cone como:

$$
\lambda K \subset K, \forall \lambda \geq 0 \text { e } K+K \subset K \text {. }
$$

Note que se $\lambda>0$ então $\lambda^{-1}>0$ e teremos

$$
\lambda \Pi^{\prime} \subset=\lambda\left(\lambda^{-1} \Pi^{\prime}\right) \subset \lambda K^{\prime} \Rightarrow \lambda K^{\prime}=K^{\prime}
$$


como também (primeira igualdade pelo acima)

$$
K+K \subset K=2 K \subset K+K \Rightarrow K+K=K .
$$

Estas duas propriedades acabam por garantir que $K$ é um cone em $X$ se

$$
0 \in K, K+K=K \quad \text { e } \quad \lambda K=K, \forall \lambda>0 .
$$

\section{Observação 2.6 (cone associado à relação de pré-ordem $\leq$ )}

Dado $(X, \leq)$ espaço vetorial pré-ordenado e $K_{0}=\{z \in X: z \geq 0\}$, note que das condições c) e d) presentes na definição de espaço vetorial pré-ordenado podemos garantir que:

- $z_{1}, z_{2} \in K_{0} \Rightarrow z_{1}+z_{2} \in K_{0}$ :

- $z \in K_{0} \Rightarrow \lambda z \in K_{0}, \forall \lambda \geq 0$.

Logo $K_{0}=\{z \geq 0\}$ é um cone em $\mathrm{X}$, denominado a partir daqui de "o cone em $X$ associado à relaçâo de pré-ordem $\leq$ dada".

\section{Definição 2.7 (cones estritos)}

Diremos que um cone $K$ em $X^{\prime}$ é estrito se $x \in K, x \neq 0 \Rightarrow-x \notin K$. Equivalentemente, $K$ é estrito se

$$
x \in K^{\prime},-x \in K^{\circ} \Rightarrow x=0 .
$$

\section{Proposição 2.8 (pré-ordem induzida por um cone)}

Considere $X$ um espaço vetorial. Uma relaçâo de pré-ordem $\leq$ compativel com as operações definidas $\epsilon m X$ é estabelecida em $X$ sempre que nele fixamos um cone $K$.

Reciprocamente, todo espaço vetorial pré-ordenado $(X, \leq)$ define um cone $K$ associado à relaçâo de pré-ordem $\leq$ dada. Neste caso, $\dot{i}$ é um cone estrito se e só se a relaçâo de pré-ordem a ele associada satisfaz a condição adicional

$$
x \leq y \leq x \Rightarrow x=y \text { (anti-simetria) }
$$

Prova: Sejam $X$ espaço vetorial e $K$ cone nele fixado. Definimos a relação de pré-ordem $\leq$ em $X$ através de $x \leq y$ se (e só se) $y-x \in K$. Teremos: 
- $x \leq x$ para todo $x \in X^{\prime}$ (uma vez que $x-x=0 \in K^{\prime}$ );

- Se $x \leq y$ e $y \leq z$ então $y-x \in K$ e $z-y \in K$, garantindo $z-x=(z-y)+(y-x) \in K$ e portanto $x \leq z$;

- Se $x \leq y$ então $y-x \in K$ e portanto para todo $z$ tem-se $(y+z)-(x+z)=y-x \in K$, garantindo $x+z \leq y+z, \forall z \in K$;

- Se $\lambda \geq 0$ então para $x \leq y$ temos $y-x \in K$ e portanto $\lambda y-\lambda x=\lambda(y-x) \in K$, garantindo $\lambda x \leq \lambda y$.

Constatamos portanto que $(X, \leq)$ é de fato um espaço vetorial préordenado.

Reciprocamente, considere $(X, \leq)$ dado. Pela propriedade $\mathrm{c}$ ) presente na definiçào de espaço vetorial pré-ordenado temos (tomando $z= \pm x$, dependendo do sentido na equivalência) :

$$
x \leq y \Leftrightarrow y-x \geq 0
$$

e, portanto, o cone $K=\{z \geq 0\}$ determina a relação de pré-ordem em $X$ uma vez que $x \leq y$ equivale a $y-x \in K$.

Supondo válida a propriedade de anti-simetria temos então que $K$ é um cone estrito $^{1}$ e, finalmente, supondo que $K$ seja um cone estrito, mostremos a validade da propriedade anti-simétrica : como $x \leq y \leq x$ implica em $y-x \in K$ e $x-y \in K$, segue $x-y=0$ como desejado.

\section{Definição 2.9 (ponto suporte cônico)}

Considere A um subconjunto fechado de um espaço de Banach $X$. Dizemos que $x_{0} \in A$ é um ponto suporte conico de $A$ se existe um cone fechado $\mathcal{C}$ de $X$ tal que

$$
A \cap\left(x_{0}+\mathcal{C}\right)=\left\{x_{0}\right\} .
$$

\footnotetext{
${ }^{1}$ De fato, usando a equivalência 2.2 no sentido " $\Leftarrow$ " temos, admitindo $K$ um cone e $x \in K^{*}$ :

- Para " $y=x$ " e " $x=0 ": x-0=x \in \mathbb{K}$ (hip.) $\Rightarrow 0 \leq x$,

- Para " $y=0$ " e " $x=x ": 0-x=-x \in K$ (hip.) $\Rightarrow x \leq 0$.

Logo $0 \leq x \leq 0$ e pela anti-simetria temos $x=0$, como desejado.
} 
Note que, em termos da pré-ordem induzida em $X$ por $\mathcal{C}$ isto significa apenas que $x_{0}$ é elemento maximal de $A$. i.e,

$$
x_{0} \leq x, x \in A \Rightarrow x=x_{0} .
$$

É verdade que. em geral, não há razão para que existam pontos suporte cônicos de um (qualquer) subconjunto fechado em um espaço de Banach. Para uma classe particular de cones, porém, a existência de tais pontos fica assegurada. Vejamos antes disso um exemplo "concreto" de cone fechado que nos será então útil.

\section{Exemplo 2.10 (o cone $\mathcal{C}(f, \gamma)$ )}

Considere $(X,\|\cdot\|)$ um espaço de Banach, $0<\gamma<1$ eff $\in \mathcal{S}_{X^{\prime}}(\|\cdot\|)$. $O$ conjunto

$$
\mathcal{C}(f, \gamma)=\{x \in X: \gamma\|x\| \leq f(x)\}
$$

define um cone fechado em X. Além disso, tal cone é gerado pelo conjunto

$$
\mathcal{B}(f, \gamma)=\{x \in X:\|x\| \leq 1 \quad \text { e } \gamma \leq f(x)\},
$$

i.e, $\mathcal{C}(f, \gamma)=[0, \infty) \cdot \mathcal{B}(f, \gamma)=\{y \in X: y=r x$ com $r \geq 0,\|x\| \leq 1, \gamma \leq$ $f(x)\}$.

\section{Prova:}

a) Escrevendo $\mathcal{C}(f, \gamma)=\mathcal{C}$, mostremos que $\mathcal{C}$ é um cone em $X$. Valendonos de (2.1). os ítens abaixo serão suficientes:

- $0 \in \mathcal{C}$. Imediato pela linearidade da $f: \gamma\|\mathbf{0}\|=0=f(\mathbf{0})$.

- $\mathcal{C}+\mathcal{C}=\mathcal{C}$. De fato, tomando $x_{1}, x_{2} \in \mathcal{C}$ temos, da desigualdade triangular e da linearidade da $f$ que

$$
\gamma\left\|x_{1}+x_{2}\right\| \leq \gamma\left\|x_{1}\right\|+\gamma\left\|x_{2}\right\| \leq f\left(x_{1}\right)+f\left(x_{2}\right)=f\left(x_{1}+x_{2}\right),
$$

garantindo assim $x_{1}+x_{2} \in \mathcal{C}$.

- $\lambda \mathcal{C}=\mathcal{C}, \forall \lambda>0$. Com efeito, supondo $x \in \mathcal{C}$ temos, para qualquer $\lambda>0$ dado, que

$$
\gamma\|\lambda x\|=\gamma \lambda\|x\| \leq \lambda f(x)=f(\lambda x),
$$

como desejado. 
b) $\mathcal{C}$ é fechado em $\mathrm{X}^{\prime}$ :

Tome qualquer sequência $\left(x_{n}\right)_{n \geq 1} \subset \mathcal{C}$ convergente para $x_{0} \in X$. Fazendo $n$ tender a infinito na desigualdade $\gamma\left\|x_{n}\right\| \leq f\left(x_{n}\right)$, válida para todo $n \geq 1$, segue pela continuidade da norma e do funcional linear $f$ que $\gamma\left\|x_{0}\right\| \leq f\left(x_{0}\right)$ e, portanto, $x_{0} \in \mathcal{C}$.

c) $\mathcal{C}(f, \gamma)=[0, \infty) \cdot \mathcal{B}(f, \gamma)$ :

Mostremos inicialmente que $\mathcal{C}(f, \gamma) \subset[0, \infty) \cdot \mathcal{B}(f, \gamma)$ :

Considere $x_{0} \in \mathcal{C}(f, \gamma)$ dado. Caso $x_{0}=\mathbf{0}$ é imediato. Do contrário consideremos $\xi=x_{0} /\left\|x_{0}\right\|$. Como $x_{0}=\left\|x_{0}\right\| \cdot \xi \operatorname{com}\|\xi\|=1$ temos

$$
\gamma\left\|x_{0}\right\| \leq f\left(\xi \cdot\left\|x_{0}\right\|\right)=\left\|x_{0}\right\| \cdot f(\xi) .
$$

Logo $\gamma \leq f(\xi)$ e segue que $x_{0} \in[0, \infty) \cdot \mathcal{B}(f, \gamma)$, como desejado.

Façamos agora a inclusão contrária: considerando $y \in[0, \infty) \cdot \mathcal{B}(f, \gamma)$, existe então $x \in X$ tal que

$$
y=r x \operatorname{com} r \geq 0,\|x\| \leq 1 \text { e } \gamma \leq f(x) .
$$

Caso $y=0$ temos $y \in \mathcal{C}(f, \gamma)$ de imediato. Do contrário $r>0$ e

$$
\gamma\|y\|=\gamma\|r x\|=\gamma r\|x\| \leq r \cdot f(x)=f(r x)=f(y),
$$

concluindo o desejado.

\section{Lema 2.11 (suficiência na existência de pontos suporte cônicos)}

Considere A um subconjunto não vazio e fechado em um espaço de Banach $(X,\|\cdot\|)$. Suponha a existência de $f \in \mathcal{S}_{X^{\prime}}(\|\cdot\|)$ tal que $f$ é limitado superiormente em $A$, i.e, $\sup _{x \in A} f(x)<\infty$.

Dados quaisquer $0<\gamma<1$ e $x \in A$, existe $x_{0} \in A$ tal que

$$
x_{0} \in x+\mathcal{C}(f, \gamma) \in A \cap\left(x_{0}+\mathcal{C}(f, \gamma)\right)=\left\{x_{0}\right\} .
$$

Em outras palavras, dados quaisquer $0<\gamma<1 \in x \in A$, sempre existe em $A$ algum ponto suporte connico $x_{0}$ associado ao cone $\mathcal{C}(f, \gamma)$ e tal que $x_{0}-x \in \mathcal{C}(f, \gamma)$. 


\section{Prova:}

Considere a sequência $\left(x_{n}\right)_{n \geq 1} \subset A$ definida indutivamente como se segue:

Tome $x_{1}=x \in A$ (dado) e considere $A_{1}=A \cap\left(x_{1}+\mathcal{C}(f, \gamma)\right)$. De $A_{1} \subset A$ e $\sup _{z \in A_{1}} f(z) \leq \sup _{z \in A} f(\tilde{z})<\infty$ temos, pela definição de supremo, que existe $x_{2} \in A_{1}$ tal que

$$
\sup _{z \in A_{1}} f(z)<f\left(x_{2}\right)+1 .
$$

Definindo $A_{2}=A \cap\left(x_{2}+\mathcal{C}(f, \gamma)\right)$, novamente sabemos existir $x_{3} \in A_{2}$ para o qual tenhamos

$$
\sup _{z \in A_{2}} f(z)<f\left(x_{3}\right)+\frac{1}{2} .
$$

Procedendo de forma análoga criamos a sequência $\left(x_{n}\right)_{n \geq 1} \subset A$ tal que, para todo $n \geq 1$,

$$
x_{n+1} \in A_{n}=A \cap\left(x_{n}+\mathcal{C}(f, \gamma)\right) \text { e } \sup _{z \in A_{n}} f(z)<f\left(x_{n+1}\right)+\frac{1}{n} .
$$

Observando agora que tem-se $x_{n+1} \in A_{n} \subset\left(x_{n}+\mathcal{C}(f, \gamma)\right)$, para todo $n \geq 1$, garante-se que

$$
\left(x_{n+1}+\mathcal{C}(f, \gamma)\right) \subset\left(x_{n}+\mathcal{C}(f, \gamma)\right)
$$

e, portanto, temos necessariamente $A_{n+1} \subset A_{n}, \forall n \geq 1$.

Considere agora qualquer $y \in A_{n+1}$ fixado. De $A_{n+1} \subset A_{n}$ garante-se que $f(y) \leq \sup _{z \in A_{n}} f(z)$ e, como $A_{n+1}=A \cap\left(x_{n+1}+\mathcal{C}(f, \gamma)\right)$. segue que $y=x_{n+1}+\xi$ onde $\xi \in \mathcal{C}(f, \gamma)$. Logo $y-x_{n+1} \in \mathcal{C}(f, \gamma)$ e então

$$
\gamma\left\|y-x_{n+1}\right\| \leq f\left(y-x_{n+1}\right)=f(y)-f\left(x_{n+1}\right) \leq \sup _{z \in A_{n}} f(z)-f\left(x_{n+1}\right)<\frac{1}{n},
$$

a última desigualdade decorrente da expressão (2.3).

Concluimos a partir daqui $\left\|z-x_{n+1}\right\|<1 /(n \cdot \gamma), \forall z \in A_{n+1}$. Logo

$$
\|z-y\| \leq\left\|z-x_{n+1}\right\|+\left\|x_{n+1}-y\right\| \leq \frac{2}{n \cdot \gamma}, \forall z, y \in A_{n+1}
$$

e como $0<\gamma<1$ está fixado, temos $\operatorname{diam}\left(A_{n+1}\right) \stackrel{n \rightarrow \infty}{\longrightarrow} 0$. 
Sendo $X$ completo sabemos, pelo teorema (1.10), que a intersecção da sequência "encaixante" $\left(A_{n}\right)_{n \geq 1}$ de fechados $\operatorname{com} \operatorname{diam}\left(A_{n}\right) \stackrel{n \rightarrow x}{\longrightarrow} 0$ consiste precisamente em um ponto. digamos $x_{0}$. De $x_{0} \in A_{1}$ temos então $x_{0} \in x_{1}+\mathcal{C}(f, \gamma)=x+\mathcal{C}(f, \gamma)$ e, uma vez que $x_{0} \in A_{n}=A \cap\left(x_{n}+\right.$ $\mathcal{C}(f, \gamma)), \forall n \geq 1$, tem-se

$$
A \cap\left(x_{0}+\mathcal{C}(f, \gamma)\right) \subset\left(A \cap\left(x_{n}+\mathcal{C}(f, \gamma)\right)\right)=A_{n}, \forall n \geq 1
$$

e portanto $A \cap\left(x_{0}+\mathcal{C}(f, \gamma)\right)=\left\{x_{0}\right\}$, encerrando a prova.

\subsection{Os teoremas de Bishop-Phelps}

\section{Teorema 2.12 (Phelps, Bronsted, Rockafellar)}

Suponha $A$ um subconjunto não vazio, convexo e fechado em um espaço de Banach $(X,\|\cdot\|) \in f \in \mathcal{S}_{X^{\prime}}(\|\cdot\|)$. Sejam $\varepsilon_{0}>0 \in \xi \in$ A tais que

$$
\sup _{z \in A} f(z) \leq f(\xi)+\varepsilon_{0} .
$$

Dado qualquer $0<\gamma<1$, existem $\varphi \in X^{\prime}$ e $\xi_{0} \in A$ tais que

$$
\sup _{z \in A} \varphi(z)=\varphi\left(\xi_{0}\right),\left\|\xi_{0}-\xi\right\| \leq \frac{\varepsilon_{0}}{\gamma} \text { e }\|\varphi-f\| \leq \gamma .
$$

Prova: Pelo lema (2.11) garante-se, em relação à $\xi \in A$, a existência de um ponto suporte cônico $\xi_{0} \in A$ associado ao cone $\mathcal{C}(f, \gamma)$ e tal que $\xi_{0} \in \xi+\mathcal{C}(f, \gamma)$.

Valendo-nos de um "argumento de separação", passaremos a obter o funcional $\varphi$ procurado que, juntamente com este $\xi_{0}$, satisfaz as demais propriedades desejadas.

Considere a aplicação $h: X \rightarrow \mathbb{R}$ definida por $h(x)=\gamma\|x\|-f(x)$, e a epígrafe dela, definida como sendo

$$
\operatorname{epi}(h)=\{(x, t) \in \mathbb{X} \times \mathbb{R}: h(x) \leq t\} .
$$

Seja também

$$
A_{1}=\left\{(z, 0) \in X \times \mathbb{R}: \xi_{0}+z \in A\right\} .
$$

Passamos então a verificar as hipóteses necessárias à aplicação do teorema (1.14). 
- epi(h) é subconjunto sólido e convexo do espaço de Banach $X \times \mathbb{R}$.

Façamos por partes:

a) epi(h) é sólido, i.e, $\operatorname{int}(\operatorname{epi}(h)) \neq \emptyset$ :

De fato, pela linearidade da $f$ garante-se que $h(0)=0, \operatorname{logo}$ $(0,1) \in \operatorname{epi}(h)$. Basta agora mostrar que $(0,1) \in \operatorname{int}(e p i(h))$.

Da continuidade de $h$ na origem (e do fato de $h(0)=0$ ) temos que, dado $0<\varepsilon<1 / 2$, existe $\delta>0$ para o qual

$$
|h(x)|<\varepsilon, \quad \forall x \in X,\|x-\mathbf{0}\|<\delta .
$$

Na notação $B_{\delta}(\mathbf{0})=\{x \in X:\|x-\mathbf{0}\|<\delta\}$ temos portanto que

$$
(\mathbf{0}, 1) \in B_{\delta}(\mathbf{0}) \times(1-\varepsilon, 1+\varepsilon) \subset \epsilon \operatorname{pi}(h),
$$

uma vez que para todo $(x, t)$ nesta vizinhança aberta de $(\mathbf{0}, 1)$ temos $h(x)<\varepsilon$ e, então, $h(x) \leq t$ para todo $t \in(1-\varepsilon, 1+\varepsilon)$. *

b) epi( $h)$ é convexo :

Sejam $0<s<1$ e quaisquer $\left(x_{1}, t_{1}\right),\left(x_{2}, t_{2}\right)$ em epi $(h)$ fixados. Devemos mostrar que $s\left(x_{1}, t_{1}\right)+(1-s)\left(x_{2}, t_{2}\right) \in e p i(h)$ i.e, que

$$
h\left(s x_{1}+(1-s) x_{2}\right) \leq s t_{1}+(1-s) t_{2} .
$$

Da desigualdade triangular e linearidade da $f$ temos

$$
\begin{array}{r}
\gamma\left\|s x_{1}+(1-s) x_{2}\right\|-f\left(s x_{1}+(1-s) x_{2}\right) \leq \\
\leq \gamma s\left\|x_{1}\right\|+(1-s) \gamma\left\|x_{2}\right\|-s f\left(x_{1}\right)-(1-s) f\left(x_{2}\right)= \\
=s\left(\gamma\left\|x_{1}\right\|-f\left(x_{1}\right)\right)+(1-s)\left(\gamma\left\|x_{2}\right\|-f\left(x_{2}\right)\right)
\end{array}
$$

e como $\left(x_{i}, t_{i}\right) \in \operatorname{epi}(h)$ nos garante que $h\left(x_{i}\right) \leq t_{i}(i=1,2)$, temos então

$$
\gamma\left\|x_{i}\right\|-f\left(x_{i}\right) \leq t_{i} \quad(i=1,2) .
$$

Segue da expressão (2.4) que

$$
h\left(s x_{1}+(1-s) x_{2}\right) \leq s t_{1}+(1-s) t_{2}
$$

como desejado. 
- $A_{1}$ é subconjunto nào-vazio e convexo de $X \times \mathbb{R}$ :

De fato. como $(0,0)$ é (trivialmente) elemento de $A_{1}$, certamente $A_{1} \neq \emptyset$.

Considerando agora quaisquer $0<t<1$ e $\left(z_{1}, 0\right),\left(z_{2}, 0\right)$ pontos fixados em $A_{1}$, devemos mostrar que $t\left(z_{1}, 0\right)+(1-t)\left(z_{2}, 0\right) \in A_{1}$ ou, equivalentemente, garantir que $\xi_{0}+\left(t z_{1}+(1-t) z_{2}\right) \in A$.

Como $\xi_{0}+\tilde{z}_{1}$ e $\xi_{0}+z_{2}$ estão em $A$ (pois $\left(z_{i}, 0\right) \in A_{1}, i=1,2$ ) segue da convexidade dele que

$$
t\left(\xi_{0}+z_{1}\right)+(1-t)\left(\xi_{0}+z_{2}\right)=\xi_{0}+t z_{1}+(1-t) z_{2} \in A,
$$

como queríamos.

- $A_{1} \cap \operatorname{int}(\operatorname{epi}(h))=\emptyset$ :

Será suficiente mostrarmos que

$$
A_{\mathbf{1}} \cap \operatorname{epi}(h)=\{(\mathbf{0}, 0)\} \quad \text { e }(\mathbf{0}, 0) \in \partial(\operatorname{epi}(h))
$$

onde $\partial(\epsilon p i(h))$ denota a fronteira de $\operatorname{epi}(h)$ em $\mathrm{X} \times \mathbb{R}$.

Uma vez que não há elemento de $A_{1}$ cuja segunda coordenada seja não-nula, um elemento qualquer de $A_{1} \cap \operatorname{epi}(h)$ pode ser expresso como $(z, 0)$ onde $\xi_{0}+z \in A$ e $h(z) \leq 0$. De $h(z)=\gamma\|z\|-f(z) \leq 0$ segue que $z \in \mathcal{C}(f, \gamma)$ e sabendo pelo lema (2.11) que

$$
A \cap\left(\xi_{0}+\mathcal{C}(f, \gamma)\right)=\left\{\xi_{0}\right\}
$$

vem (de $\xi_{0}+z \in A$ ) que, necessariamente, $z=0$. Segue portanto o desejado : $A_{1} \cap \operatorname{epi}(h)=\{(0,0)\}$, como esperado.

Justificamos $(0,0) \in \partial \operatorname{epi}(h)$ notando que $(0,0) \notin \operatorname{int}(\operatorname{epi}(h))$ : com efeito, $h(0)=0$ e $(0,-\varepsilon) \notin$ epi $(h)$, para qualquer $\varepsilon>0$ dado.

Como estamos nas condições do teorema (1.14), podemos garantir a existência de um número real $\alpha$ e um funcional nâo nulo $\psi \in(X \times \mathbb{R})^{\prime}$ tais que o hiperplano

$$
\mathcal{H}(\psi, \alpha)=\{(x, t) \in X \times \mathbb{R}: \psi(x, t)=\alpha\}
$$


separa epi(h) de $A_{1}$.

Como $(0,0) \in A_{1} \cap \epsilon$ pi(h) segue que $\alpha=0$. Supondo sem perda de generalidade $^{2}$ que epi(h) se encontra no semi-espaço $\{(x, t) \in X \times \mathbb{R}$ : $\psi(x, t) \geq 0\}$ definido pelo hiperplano $\mathcal{H}(\psi, 0)$ e, levando-se em conta que $(0,0) \in \mathcal{H}(\psi, 0) \cap\left(\right.$ epi $\left.(h) \cap A_{1}\right)$, podemos afirmar que

$$
\sup \psi\left(A_{1}\right)=0=\inf \psi(e p i(h)) .
$$

Uma vez que pelo primeiro item sabemos que $(0,1) \in \operatorname{int}(\epsilon p i(h))$ segue que $\psi(\mathbf{0}, 1) \geq 0$. Admitindo temporariamente que $\psi(\mathbf{0}, 1)>0$, o funcional não nulo $\eta=\psi / \psi(\mathbf{0}, 1) \in(X \times \mathbb{R})^{\prime}$ também satisfaz

$$
\sup \eta\left(A_{1}\right)=0=\inf \eta(\operatorname{epi}(h))
$$

e definindo a aplicação linear contínua $\varphi: X \rightarrow \mathbb{R}$ dada por $\varphi(x)=$ $\eta(x, 0), \forall x \in X$ teremos a partir de

$$
\psi(x, t)=\psi((x, 0)+(\mathbf{0}, t))=\psi(x, 0)+t \psi(\mathbf{0}, 1)
$$

que

$$
\eta(x, t)=\frac{\psi(x, 0)}{\psi(\mathbf{0}, 1)}+t=\eta(x, 0)+t=\varphi(x)+t
$$

para todo $(x, t)$ pertencente a $X \times \mathbb{R}$.

Como para qualquer $z \in A$ temos $\left(z-\xi_{0}, 0\right) \in A_{1}$ pela própria definição deste último conjunto, segue que

$$
\varphi(z)-\varphi\left(\xi_{0}\right)=\varphi\left(z-\xi_{0}\right)=\eta\left(z-\xi_{0}, 0\right) \leq 0,
$$

donde podemos concluir que

$$
\sup _{z \in A} \varphi(z) \leq \varphi\left(\xi_{0}\right) .
$$

Como trivialmente $(x, h(x)) \in$ epi(h) para todo $x \in X$, então

$$
0 \leq \eta(x, h(x))=\varphi(x)+h(x)=\varphi(x)+\gamma \cdot\|x\|-f(x)
$$

\footnotetext{
${ }^{2}$ Vide observação que segue a definição (1.13).
} 
o que nos leva a concluir, como desejado, que

$$
\|f-\varphi\| \leq \gamma
$$

uma vez que

$$
\sup _{x \in \mathcal{S}_{X}(\|\cdot\|)}|f(x)-\varphi(x)| \leq \gamma
$$

Pelo primeiro parágrafo desta prova temos que $\xi_{0}-\xi \in \mathcal{C}(f, \gamma)$, garantindo assim

$$
\gamma\left\|\xi_{0}-\xi\right\| \leq f\left(\xi_{0}-\xi\right)=f\left(\xi_{0}\right)-f(\xi) \leq \sup _{z \in A} f(z)-f(\xi) \leq \varepsilon_{0},
$$

valendo a última desigualdade pela hipótese dada. Segue portanto desta expressão que $\left\|\xi_{0}-\xi\right\| \leq \varepsilon_{0} / \gamma$, como desejado.

Resta-nos apenas verificar o postergado, a saber: $\psi(0,1)>0$.

De fato, pelo primeiro item sabemos que dado qualquer $0<\varepsilon<1 / 2$ existe $\delta>0$ para o qual

$$
(0,1) \in B_{\delta}(0) \times(1-\varepsilon, 1+\varepsilon) \subset \operatorname{epi}(h) .
$$

Denotando $B_{\delta}(\mathbf{0}) \times(1-\varepsilon, 1+\varepsilon)$ por $\Omega$, note que $\psi$ restrita à $\Omega$ não é identicamente nula (do contrário a linearidade de $\psi$ garantiria $\psi=0$, absurdo) e por isso existe (pelo menos) um ponto $\left(x_{p}, t_{p}\right) \in \Omega$ tal que $\psi\left(x_{p}, t_{p}\right)>0$ (uma vez que $\Omega \subset$ epi $(h)$ ).

Por linearidade temos então

$$
0<\psi\left(x_{p}, t_{p}\right)=\psi\left(x_{p}, 0\right)+t_{p} \cdot \psi(0,1) .
$$

Caso (possamos tomar) $x_{p}=0$ segue que sendo $t_{p}>0$ (pois $t_{p} \in$ $(1-\varepsilon, 1+\varepsilon)$ para $0<\varepsilon<1 / 2)$ temos $\psi(0,1)>0$ e nada resta fazer. Do contrário temos $x_{p} \neq 0$ e observando que $\left(-x_{p}, t_{p}\right) \in \Omega$ podemos pela expressão (2.5) afirmar que

$$
0<-\psi\left(x_{p}, 0\right)+t_{p} \cdot \psi(\mathbf{0}, 1) \text {. }
$$

Concluimos a partir destas desigualdades que

$$
-t_{p} \cdot \psi(0,1)<\psi\left(x_{p}, 0\right)<t_{p} \cdot \psi(0,1)
$$


e daqui tem-se trivialmente que $\psi(0,1)=0$ é impossivel.

Uma vez que este era o último detalhe a ser provado damos por encerrada a prova do lema.

\section{Observação 2.13 (generalizando o teorema do suporte)}

É interessante comentar que este teorema admite como corolário o chamado "primeiro teorema de Bishop-Phelps", que trata da densidade dos pontossuporte de um subconjunto convexo e fechado num espaço de Banach $X$. Trata-se de um resultado associado ao teorema do suporte (corolário (1.15)) e pode ser enunciado como se segue.

\section{Teorema 2.14 (o primeiro teorema de Bishop-Phelps)}

Os pontos-suporte (de hiperplanos) de um subconjunto convexo e fechado em um espaço de Banach constituem um conjunto denso na fronteira deste subconjunto.

Prova: Vide [Holmes], 166.

Passamos agora a tentar responder a respeito da densidade $\left(\mathrm{em} X^{\prime}\right)$ dos funcionais lineares contínuos que atingem suas respectivas normas.

\section{Teorema 2.15 (o segundo teorema de Bishop-Phelps)}

Sejam dados $A$ um subconjunto não vazio, convexo e fechado num espaço de Banach $(X,\|\cdot\|)$ e $f \in \mathcal{S}_{X^{\prime}}(\|\cdot\|)$ limitado superiormente em $A$.

Para qualquer $0<\delta<1$, existe um funcional nào nulo $\psi \in X^{\prime \prime}$ que atinge seu supremo ${ }^{3}$ em $A$ e é tal que $\|f-\psi\|<,\delta$.

Prova: Tome $\xi \in A$ tal que

$$
\sup _{z \in A} f(z) \leq f(\xi)+1
$$

\footnotetext{
${ }^{3}$ Isto é, existe $\xi_{0} \in A$ para o qual
}

$$
\sup _{z \in A} \psi(z)=\psi\left(\xi_{0}\right)
$$


Para qualquer $0<\delta<1$ fixado, o teorema (2.12) garante existirem $\psi \in X^{\prime \prime}$ e $\xi_{0} \in A$ tais que

$$
\sup _{z \in A} \psi(z)=\psi\left(\xi_{0}\right) \quad \text { e } \quad\|f-\psi\| \leq \delta<1=\|f\| .
$$

Como $\|f\|-\|\psi\|<\|f\|$. então $\psi \neq \equiv 0$.

\section{Corolário 2.16}

Seja A um subconjunto nâo vazio, convexo, fechado e limitado num espaço de Banach $(X,\|\cdot\|)$. O subconjunto de $X^{\prime}$, constituido pelos funcionais lineares e contínuos que atingem seus supremos em $A$ é denso em $X^{\prime \prime}$.

Prova: Seja $f \in X^{\prime \prime}$. Se $f \equiv 0$ nada resta a fazer. Do contrário considere fixado $0<\varepsilon<\|f\|$ e tome $g \in \mathcal{S}_{X^{\prime}}(\|\cdot\|)$ dado por $g=f /\|f\|$. Como $A$ é limitado segue que $\sup _{z \in A} g(z)<\infty$ e pelo teorema (2.15) garantimos a existência de $\psi \in X^{\prime \prime}$ nào nulo que atinge seu supremo em $A$ e satisfaz $\|g-\psi\|<\varepsilon /\|f\|$.

Finalmente, o funcional $\varphi=\|f\| \cdot \psi \in X^{\prime}$ atinge seu supremo em $A$ e é tal que

$$
\|\varphi-f\|=\|\| f\|\cdot \psi-g \cdot\| f\|\|=\|f\| \cdot\|\psi-g\|<\|f\| \frac{\varepsilon}{\|f\|}=\varepsilon,
$$

garantindo assim a densidade procurada.

\section{Corolário 2.17 (o clássico teorema de Bishop-Phelps)}

Todo espaço de Banach é subreflexivo.

Prova: Basta observar que a bola unitária $\mathcal{B}_{X}(\|\cdot\|)$ constitui um subconjunto convexo, limitado e fechado em $X$. Neste caso em particular, uma vez fixado $f \in X^{\prime}, f$ atingir seu supremo em $\mathcal{B}_{X}(\|\cdot\|)$ significa, por definição, atingir sua norma. O resultado se obtém, portanto, como aplicação imediata do corolário anterior. 


\section{Capítulo 3}

\section{Alguns resultados sobre operadores entre espaços de Banach que atingem suas normas}

\subsection{Introdução}

Bishop e Phelps (em [B.P.]) forneceram a primeira demonstração da prova do teorema que recebeu seus nomes e, no mesmo artigo, sugerem um problema interessante: quais espaços de Banach poderiam ocupar o "papel" do corpo de escalares no resultado por eles obtido? Antes de formalizar esta pergunta, passamos a definir o espaço dos operadores "norm-attaining" $\mathcal{N} \mathcal{A}(X, Y)$, de grande importância durante toda a continuação da dissertação.

Definição 3.1 (o espaço $\mathcal{N} \mathcal{A}\left(X^{r}, Y^{r}\right)$ )

Sejam $X$ e $Y$ espaços de Banach e $\mathcal{L}(X, Y)$ o espaço dos operadores lineares e limitados de $X$ em $Y$.

Definimos o subespaço $\mathcal{N} \mathcal{A}(X, Y) \subset \mathcal{L}(X, Y)$ como sendo dado pelos operadores que atingem suas respectivas normas, i.e,

$$
T \in \mathcal{L}(X, Y) \text { tal que } \exists x_{0} \in \mathcal{S}_{X}(\|\cdot\|) \text { tal que }\left\|T\left(x_{0}\right)\right\|=\|T\| .
$$

Podemos então reapresentar a questão proposta pelos matemáticos: 
Problema: Sendo $X$ e $Y$ espaços de Banach, o que se pode afirmar quanto à densidade de $\Upsilon^{\mathcal{A}} \mathcal{A}(X, Y)$ em $\mathcal{L}(X, Y)$ ?

J. Lindenstrauss foi o primeiro autor ${ }^{1}$ a estudar esta questào de forma sistemática. No seu artigo ([Lindenst], 1963) ele prova que. em geral, $\mathcal{N} \mathcal{A}(X, Y)$ nào é denso no $\mathcal{L}\left(X^{\prime} Y\right)$ e introduz as (a partir de então) chamadas "propriedades $A$ e $B$ " (o faremos mais adiante) que visam facilitar a abordagem do problema que, segundo ele, "parece muito geral para admitir uma solução completa" ${ }^{2}$.

Passemos agora a definir out ro subespaço de $\mathcal{L}(X, Y)$ que terá papel fundamental no expressivo lema técnico de Lindenstrauss que o sucede.

\section{Definição 3.2 (o espaço $\mathcal{F}_{0}\left(X^{\prime}, Y^{\prime}\right)$ )}

Sejam $(X,\|\cdot\|)$ e $(Y,\|\cdot\|)$ espaços de Banach. O subespaço constituido pelos operadores $T \in \mathcal{L}(X, Y)$ cujos bi-adjuntos $T^{\prime \prime} \in \mathcal{L}\left(X^{\prime \prime}, Y^{\prime \prime}\right)$ atingem suas respectivas normas será denotado por $\mathcal{P}_{0}(X, Y)$.

\subsection{Primeiros resultados}

\section{Lema 3.3}

Sejam $X^{\prime}$ e $Y$ espaços de Banach. Um operador $T \in \mathcal{L}(X, Y)$ pertence ao espaço $\mathcal{P}_{0}(X, Y)$ se $e$ somente se existem sequências $\left(x_{k}\right)_{k \geq 1} \subset X^{\prime} e$ $\left(f_{k}\right)_{k \geq 1} \subset Y^{\prime}$ tais que

a) $\left\|x_{k}\right\|=\left\|f_{k}\right\|=1, \forall k \geq 1 \quad \epsilon$

b) $\left|f_{j}\left(T x_{k}\right)\right| \geq\|T\|-\frac{1}{j}, \forall k \geq 2, \forall 1 \leq j<k$.

Prova: Consideraremos $T \neq 0$ (do contrário o resultado é imediato).

Façamos inicialmente $(\Rightarrow): \operatorname{sejam} T \in \mathcal{P}_{0}(X, Y)$ e $\varphi \in \mathcal{S}_{X^{\prime \prime}}(\|\cdot\|)$ tais que $\left\|T^{\prime \prime} \varphi\right\|=\left\|T^{\prime \prime}\right\|$.

\footnotetext{
${ }^{1}$ Segundo Maria D.Acosta, que também resolve questões associadas, como veremos no capítulo seguinte.

${ }^{2}$ Extraido do [Lindenst]
} 
Uma vez que o teorema (1.2S) nos garante que $\left\|T^{\prime \prime}\right\|=\|T\|$, então, da definição de $\left\|T^{\prime \prime} \varphi\right\|$, garantimos existir uma sequência $\left(f_{j}\right)_{j \geq 1}$ tal que

$$
\left(f_{j}\right)_{j \geq 1} \subset \mathcal{S}_{Y^{\prime}}(\|\cdot\|) \quad \text { e } \quad\left|\left(T^{\prime \prime} \varphi\right)\left(f_{j}\right)\right| \geq\|T\|-\frac{1}{2 j}, \forall j \geq 1 .
$$

Sendo $J$ a imersão canônica de $X^{\prime}$ em $X^{\prime \prime}$, o teorema de Goldstine(1.32) nos assegura que $J\left(\mathcal{B}_{X}(\|\cdot\|)\right)$ é fraca-estrela densa em $\mathcal{B}_{X^{\prime \prime}}(\|\cdot\|)$ e, portanto, existe ponto de $J\left(\mathcal{B}_{X}(\|\cdot\|)\right)$ próximo de y em relação às vizinhanças (tomemos básicas) dadas pela topologia $\sigma\left(X^{\prime \prime}, X^{\prime \prime}\right)$. Garante-se assim que, para cada $k \geq 2$,

$$
\exists y_{k} \in \mathcal{B}_{X}(\|\cdot\|) \quad \text { tal que } \sup _{1 \leq j<k}\left|\left(J\left(y_{k}\right)-\vartheta\right)\left(T^{\prime} f_{j}\right)\right|<\frac{1}{2 k} .
$$

Note que sendo $J$ isometria e $\varphi$ não nulo, $J\left(y_{k}\right) \in J\left(\mathcal{B}_{X}(\|\cdot\|)\right)$ próximo de $\varphi$ nos permite admitir, sem perda de generalidade, que $y_{k} \neq 0$ para todo $k \geq 1$. Definindo então $x_{k}=y_{k} /\left\|y_{k}\right\|$, teremos

$$
\begin{aligned}
\sup _{1 \leq j<k}\left|\left(J\left(\left\|y_{k}\right\| \cdot x_{k}\right)-\varphi\right)\left(T^{\prime} f_{j}\right)\right| & =\sup _{1 \leq j<k}\left|\left(T^{\prime} f_{j}\right)\left(\left\|y_{k}\right\| \cdot x_{k}\right)-\varphi\left(T^{\prime} f_{j}\right)\right|= \\
& =\sup _{1 \leq j<k}\left|\left\|y_{k}\right\|\left(T^{\prime} f_{j}\right)\left(x_{k}\right)-\varphi\left(T^{\prime} f_{j}\right)\right|<\frac{1}{2 k} .
\end{aligned}
$$

Como

$$
\begin{aligned}
\left|\varphi\left(T^{\prime} f_{j}\right)\right| & =\left|\varphi\left(T^{\prime} f_{j}\right)-\left\|y_{k}\right\|\left(T^{\prime} f_{j}\right)\left(x_{k}\right)+\left\|y_{k}\right\|\left(T^{\prime} f_{j}\right)\left(x_{k}\right)\right| \leq \\
& \leq\left|\varphi\left(T^{\prime} f_{j}\right)-\left\|y_{k}\right\|\left(T^{\prime} f_{j}\right)\left(x_{k}\right)\right|+\left\|y_{k}\right\|\left|\left(T^{\prime} f_{j}\right)\left(x_{k}\right)\right|,
\end{aligned}
$$

temos que

$$
\left|\varphi\left(T^{\prime} f_{j}\right)\right|<\frac{1}{2 k}+\left\|y_{k}\right\|\left|\left(T^{\prime} f_{j}\right)\left(x_{k}\right)\right| \leq \frac{1}{2 k}+\left|\left(T^{\prime} f_{j}\right)\left(x_{k}\right)\right|
$$

para cada $1 \leq j<k$, uma vez que $\left\|y_{k}\right\| \leq 1$ para todo $k \geq 2$.

Pelas expressões (3.1) e anterior teremos, para todo $1 \leq j<k$,

$$
\|T\|-\frac{1}{2 j} \leq\left|\varphi\left(T^{\prime} f_{j}\right)\right|<\frac{1}{2 k}+\left|\left(T^{\prime} f_{j}\right)\left(x_{k}\right)\right| .
$$


Finalmente podemos concluir que

$$
\left|\left(T^{\prime} f_{j}\right)\left(x_{k}\right)\right|>\|T\|-\frac{1}{2 j}-\frac{1}{2 k} \geq\|T\|-\frac{1}{2 j}-\frac{1}{2 j}=\|T\|-\frac{1}{j},
$$

garantindo então o que faltava :

$$
\left|\left(T^{\prime} f_{j}\right)\left(x_{k}\right)\right|>\|T\|-\frac{1}{j}, \forall k \geq 2, \forall 1 \leq j<k .
$$

Passamos agora a provar a recíproca : suponha dados $T \in \mathcal{L}(X, Y)$ e as sequências $\left(x_{k}\right)_{k \geq 1},\left(f_{k}\right)_{k \geq 1}$ nas condições consideradas. Sem perda de generalidade admitiremos que $\|T\|=1$.

Tome o subespaço de $X^{\prime}$ gerado pelo conjunto $\left\{T^{\prime} f_{k}\right\}_{k>1}$ e seja $\Gamma^{\prime}$ o fecho deste subespaço na topologia da norma. Sendo $\Gamma^{\prime}$ um espaço de Banach separável, o teorema (1.31) nos garante que a bola $\mathcal{B}_{\Gamma \prime}(\|\cdot\|)$ é fraca-estrela metrizável.

Uma vez que $X^{\prime \prime} \subset \Gamma^{\prime \prime}$ e $J$ é uma isometria, $\left(J\left(x_{k}\right)\right)_{k \geq 1} \subset \mathcal{B}_{\Gamma^{\prime \prime}}(\|\cdot\|)$. Assim sendo, a sequência $\left(J\left(x_{k}\right)\right)_{k \geq 1}$ admite uma subsequência fraca-estrela convergente para (digamos) $x_{0}^{\prime \prime} \in \mathcal{B}_{\Gamma^{\prime \prime}}(\|\cdot\|)$. Como $x_{0}^{\prime \prime}: \Gamma^{\prime} \rightarrow \mathbb{R}$ é um funcional linear contínuo de norma um, o teorema (1.18) nos permite considerar a extensão $\widetilde{x_{0}^{\prime \prime}}: X^{\prime} \rightarrow \mathbb{R}$ de mesma norma para concluir que $\widetilde{x_{0}^{\prime \prime}} \in \mathcal{B}_{X^{\prime \prime}}(\|\cdot\|)$.

Fixado qualquer $\varepsilon>0$, pela convergência fraca-estrela podemos garantir que dada uma vizinhança fraca-estrela qualquer de $\widetilde{x_{0}^{\prime \prime}}$

$$
V_{\widetilde{x_{0}^{\prime \prime}}}=\left\{F \in X^{\prime \prime}: \sup _{j \in I}\left|\left(F-\widetilde{x_{0}^{\prime \prime}}\right)\left(T^{\prime} f_{j}\right)\right|<\varepsilon\right\}
$$

com $I=\left\{1,2, \ldots, n_{0}\right\} \quad\left(n_{0} \geq 1\right)$, existe $k_{0} \geq 2$ tal que

$$
\sup _{j \in I}\left|\left(J\left(x_{k}\right)-\tilde{x_{0}^{\prime \prime}}\right)\left(T^{\prime} f_{j}\right)\right|<\varepsilon, \quad \forall k \geq k_{0} \text {. }
$$

Uma vez que $\tilde{x_{0}^{\prime \prime}}$ restrito à $\Gamma^{\prime}$ coincide com $x_{0}^{\prime \prime}$, para todo $k \geq 2 \mathrm{e}$ qualquer $1 \leq j<k$ temos, pela hipótese e desigualdade triangular, que

$$
\|T\|-\frac{1}{j}-\left|x_{0}^{\prime \prime}\left(T^{\prime} f_{j}\right)\right| \leq\left|f_{j}\left(T x_{k}\right)\right|-\left|x_{0}^{\prime \prime}\left(T^{\prime} f_{j}\right)\right| \leq\left|f_{j}\left(T x_{k}\right)-x_{0}^{\prime \prime}\left(T^{\prime} f_{j}\right)\right| .
$$


Considerando $k_{1}=\max \left\{n_{0}, k_{0}\right\}$ temos, pela expressào (3.2), que

$$
\left|f_{j}\left(T x_{k}\right)-x_{0}^{\prime \prime}\left(T^{\prime} f_{j}\right)\right|<\varepsilon
$$

para todo $j \in I$ e todo $k \geq k_{1}$. Segue nestas condições que, pelas desigualdades anteriores, $\left|x_{0}^{\prime \prime}\left(T^{\prime} f_{j}\right)\right|>\|T\|-\frac{1}{j}-\varepsilon, \forall j \in I$.

Como podemos escolher $\varepsilon>0$ arbitrariamente pequeno e $n_{0}$ arbitrariamente grande, fazendo $j \rightarrow \infty$ segue que

$$
\left\|T^{\prime \prime}\right\| \geq\left\|T^{\prime \prime} x_{0}^{\prime \prime}\right\| \geq\left|\left(T^{\prime \prime} x_{0}^{\prime \prime}\right)\left(f_{j}\right)\right|=\left|x_{0}^{\prime \prime}\left(T^{\prime} f_{j}\right)\right| \stackrel{j \rightarrow \infty}{\longrightarrow}\|T\|=\left\|T^{\prime \prime}\right\|
$$

garantindo que $T^{\prime \prime}$ atinge sua norma e, assim sendo, $T \in \mathcal{P}_{0}(X, Y)$.

\section{Observação 3.4}

Antes de apresentar o próximo teorema relacionado com o lema (3.3), mostremos que, fixado $0<\varepsilon<1 / 3$, existe uma sequência decrescente de números reais positivos $\left(\varepsilon_{k}\right)_{k \geq 1}$ que satisfaz :

$$
2 \sum_{k \geq 1} \varepsilon_{k}<\varepsilon \quad ; \quad 2 \sum_{i \geq k+1} \varepsilon_{i}<\varepsilon_{k}^{2} \quad \text { e } \quad \varepsilon_{k}<\frac{1}{10 k} \quad, \forall k \geq 1 .
$$

De fato, tome inicialmente $\varepsilon_{p}=(\varepsilon / 10)^{p}, \forall p \geq 1$. Obviamente temos

- $2 \sum_{p \geq 1} \varepsilon_{p}=2 \sum_{p \geq 1}\left(\frac{\varepsilon}{10}\right)^{p}=2 \frac{\frac{\varepsilon}{10}}{1-\frac{\varepsilon}{10}}=\frac{2 \varepsilon}{10-\varepsilon}<\frac{2 \varepsilon}{9}<\varepsilon \quad$ e

- Para qualquer $p \geq 1$,

$$
\varepsilon_{p}=\left(\frac{\varepsilon}{10}\right)^{p}<\frac{1}{10^{p}} \leq \frac{1}{10 p}
$$

uma vez que $0<\varepsilon<1 / 3$.

Passamos a exibir uma subsequência $\left(\varepsilon_{p_{j}}\right)_{j \geq 1}$ de $\left(\varepsilon_{p}\right)_{p \geq 1}$ que satisfaz inclusive a segunda desigualdade requerida $e$, portanto, constitui a (uma possível) sequência desejada.

- Definimos $\left(\varepsilon_{p_{j}}\right)_{j \geq 1} \subset\left(\varepsilon_{p}\right)_{p \geq 1}$ com $p_{1}=1$ e $p_{j}=1+2 \cdot p_{j-1}$, para todo $j \geq 2$. 
Temos então, para todo $j \geq 1$,

$$
\begin{aligned}
2 \sum_{i \geq j+1} \varepsilon_{p_{t}} & =2 \sum_{i \geq j+1}\left(\frac{\Xi}{10}\right)^{p_{i}}<2 \sum_{\substack{k \geq 0 \\
\varepsilon^{p_{j+1}}}}\left(\frac{\Xi}{10}\right)^{k+p_{\jmath+1}}= \\
& =2 \frac{\left(\frac{\varepsilon}{10}\right)^{p_{\jmath+1}}}{1-\frac{\varepsilon}{10}}=2 \frac{2}{10-\varepsilon}<\frac{\varepsilon^{p_{\jmath+1}}}{9} \frac{10^{p_{\jmath+1}-1}}{10-1}
\end{aligned}
$$

Como $p_{j+1}=2 p_{j}+1$, então $10^{p_{j+1}-1}=10^{2} p_{j}$ e, como desejado

$$
2 \sum_{i \geq j+1} \varepsilon_{p_{i}}<\frac{2}{9} \frac{\varepsilon^{p_{\jmath+1}}}{10^{p_{\jmath+1}-1}}=\frac{2}{9} \frac{\varepsilon^{p_{\jmath+1}}}{10^{2 p_{\jmath}}}<\left(\frac{\varepsilon}{10}\right)^{2 p_{\jmath}}=\varepsilon_{p_{\jmath}}^{2},
$$

valendo a última desigualdade como decorrência trivial de $(2 \varepsilon) / 9<1$. *

Passamos a provar um lema técnico de grande importância para o teorema de Lindenstrauss que o sucede.

\section{Lema 3.5}

Sejam $X, Y$ espaços de Banach, $0<\varepsilon<1 / 3$ e $T \in \mathcal{L}(X, Y)$ (não nulo) dados. Existem $\left(x_{k}\right)_{k \geq 1} \subset X,\left(f_{k}\right)_{k \geq 1} \subset Y^{\prime} \in\left(T_{k}\right)_{k \geq 1} \subset \mathcal{L}\left(X^{\prime}, Y\right)$ que cumprem as seguintes propriedades:

$$
\begin{gathered}
T_{1}=T \\
\left\|T_{k} x_{k}\right\| \geq\left\|T_{k}\right\|-\varepsilon_{k}^{2} \quad \operatorname{com}\left\|x_{k}\right\|=1, \forall k \geq 1 ; \\
f_{k}\left(T_{k} x_{k}\right)=\left\|T_{k} x_{k}\right\| \quad \operatorname{com}\left\|f_{k}\right\|=1, \forall k \geq 1 ; \\
T_{k+1}(x)=T_{k}(x)+\varepsilon_{k} \cdot f_{k}\left(T_{k} x\right) \cdot\left(T_{k} x_{k}\right), \forall x \in X, \forall k \geq 1 . \\
\left\|T_{j}-T_{k}\right\| \leq 2 \sum_{i=j}^{k-1} \varepsilon_{i}, \quad \operatorname{com}\left\|T_{k}\right\| \leq 4 / 3 \quad, \forall 1 \leq j \leq k-1, \forall k \geq 2 . \\
\left|f_{j}\left(T_{j} x_{k}\right)\right| \geq\left\|T_{j}\right\|-6 \varepsilon_{j} \quad, \forall 1 \leq j \leq k-1, \forall k \geq 2 .
\end{gathered}
$$

onde $\left(\varepsilon_{k}\right)_{k \geq 1}$ é uma sequência decrescente de números reais que satisfaz as condiçôes presentes em (3.3). 
Prova: Dados $0<\varepsilon<1 / 3$ e $T \in \mathcal{L}(X, Y$ ) com (sem perda de generalidade) $\|T\|=1$, tome uma sequência $\left(\Sigma_{k}\right)_{k \geq 1}$ que satisfaz as desigualdades dadas em (3.3) .

Construiremos indutivamente as sequências $\left(T_{k}\right)_{k \geq 1} \subset \mathcal{L}(X, Y)$. $\left(x_{k}\right)_{k \geq 1} \subset X$ e $\left(f_{k}\right)_{k \geq 1} \subset Y^{\prime}$ de forma a satisfazer as expressões (3.4). (3.5), (3.6) e (3.7). A seguir, provaremos que elas também satisfazem as desigualdades das duas últimas expressões.

Para isso observe que, de imediato, defino $T_{1}: X \rightarrow Y$ como sendo igual a $T$ e sendo $\varepsilon_{1}^{2}>0$, segue da definiçào de $\left\|T_{1}\right\|$ que

$$
\exists x_{1} \in \mathcal{S}_{X}(\|\cdot\|) \text { tal que }\left\|T_{1} x_{1}\right\| \geq\left\|T_{1}\right\|-\varepsilon_{1}^{2} .
$$

Pelo teorema (1.17), para $T_{1} x_{1} \in Y$ existe $f_{1} \in \mathcal{S}_{Y^{\prime}}(\|\cdot\|)$ tal que $f_{1}\left(T_{1} x_{1}\right)=\left\|T_{1} x_{1}\right\|$.

Defino $T_{2}: X \rightarrow Y$ por $T_{2}(x)=T_{1}(x)+\varepsilon_{1} \cdot f_{1}\left(T_{1} x\right) \cdot\left(T_{1} x_{1}\right), \forall x \in X$ (obviamente linear e contínua) e, sendo $\varepsilon_{2}^{2}>0$, segue da definição de $\left\|T_{2}\right\|$ que

$$
\exists x_{2} \in \mathcal{S}_{X}(\|\cdot\|) \text { tal que }\left\|T_{2} x_{2}\right\| \geq\left\|T_{2}\right\|-\varepsilon_{2}^{2} .
$$

Novamente existe $f_{2} \in \mathcal{S}_{Y^{\prime \prime}}(\|\cdot\|)$ tal que $f_{2}\left(T_{2} x_{2}\right)=\left\|T_{2} x_{2}\right\|$. Assim fazendo, construimos as sequências desejadas.

Passamos agora à prova da desigualdade (3.8), que será repetida por conveniência.

$$
\left\|T_{j}-T_{k}\right\| \leq 2 \sum_{i=j}^{k-1} \varepsilon_{i}, \text { com }\left\|T_{k}\right\| \leq 4 / 3 \quad, \forall 1 \leq j \leq k-1, \forall k \geq 2 .
$$

Façamos por indução em $k$ :

Caso $k=2$ (portanto $j=1$ ): devemos mostrar que

$$
\left\|T_{1}-T_{2}\right\| \leq 2 \varepsilon_{1} \quad \text { e } \quad\left\|T_{2}\right\| \leq 4 / 3 .
$$

Para todo $x \in X$ temos

$$
\begin{aligned}
\left\|\left(T_{1}-T_{2}\right)(x)\right\| & =\left\|\varepsilon_{1} \cdot f_{1}\left(T_{1} x\right) \cdot\left(T_{1} x_{1}\right)\right\|=\varepsilon_{1} \cdot\left|f_{1}\left(T_{1} x\right)\right| \cdot\left\|T_{1} x_{1}\right\| \leq \\
& \leq \varepsilon_{1} \cdot\left|f_{1}\left(T_{1} x\right)\right| \cdot\left\|T_{1}\right\|=\varepsilon_{1} \cdot\left|f_{1}\left(T_{1} x\right)\right|
\end{aligned}
$$


uma vez que $\left\|x_{1}\right\|=\left\|T_{1}\right\|=\|T\|=1$.

Dado qualquer $x \in \mathcal{S}_{X}(\|\cdot\|)$ podemos então concluir que

$$
\left\|\left(T_{1}-T_{2}\right)(x)\right\| \leq \varepsilon_{1} \cdot\left\|f_{1}\right\| \cdot\left\|T_{1}\right\| \cdot\|x\|=\varepsilon_{1},
$$

uma vez que $\left\|f_{1}\right\|=\left\|T_{1}\right\|=\|x\|=1$ e. assim sendo,

$$
\left\|T_{1}-T_{2}\right\| \leq \varepsilon_{1}<2 \varepsilon_{1}
$$

como desejado. Além disso temos, de imediato,

$$
\left\|T_{2}\right\| \leq\left\|T_{2}-T_{1}\right\|+\left\|T_{1}\right\|<2 \varepsilon_{1}+1<\varepsilon+1<\frac{1}{3}+1=\frac{4}{3},
$$

a outra desigualdade almejada.

Suponhamos por hipótese de indução que para $k>2$ fixado e qualquer $1 \leq j \leq k-2$ tenhamos $\left\|T_{j}-T_{k-1}\right\| \leq 2 \sum_{i=j}^{k-2} \varepsilon_{i}$ e $\left\|T_{k-1}\right\| \leq 4 / 3$.

Como $\left\|T_{j}-T_{k}\right\| \leq\left\|T_{j}-T_{k-1}\right\|+\left\|T_{k-1}-T_{k}\right\|$, segue da hipótese de indução que

$$
\left\|T_{j}-T_{k}\right\| \leq 2 \sum_{i=j}^{k-2} \varepsilon_{i}+\left\|T_{k-1}-T_{k}\right\|,
$$

para todo $1 \leq j \leq k-2$.

Temos também

$$
\begin{aligned}
\left\|\left(T_{k-1}-T_{k}\right)(x)\right\| \stackrel{(3.7)}{=}\left\|\varepsilon_{k-1} \cdot f_{k-1}\left(T_{k-1} x\right) \cdot\left(T_{k-1} x_{k-1}\right)\right\| \leq \\
\leq \varepsilon_{k-1} \cdot\left|f_{k-1}\left(T_{k-1} x\right)\right| \cdot\left\|T_{k-1}\right\| \cdot \overbrace{\mid x_{k-1} \|}^{=1} \leq \\
\leq \varepsilon_{k-1} \cdot \underbrace{\left\|f_{k-1}\right\|}_{=1} \cdot\left\|T_{k-1}\right\|^{2} \cdot\|x\|,
\end{aligned}
$$

garantindo assim para todo $x \in \mathcal{S}_{X}(\|\cdot\|)$ que

$$
\left\|\left(T_{k-1}-T_{k}\right)(x)\right\| \leq \varepsilon_{k-1} \frac{16}{9} .
$$

Portanto

$$
\left\|T_{j}-T_{k}\right\| \leq 2 \sum_{i=j}^{k-2} \varepsilon_{i}+\frac{16}{9} \varepsilon_{k-1}<2 \sum_{i=j}^{k-2} \varepsilon_{i}+2 \varepsilon_{k-1}=2 \sum_{i=j}^{k-1} \varepsilon_{i}
$$


como desejado, restando mostrar que $\left\|T_{k}\right\| \leq 4 / 3$. Isto se dá pois

$$
\left\|T_{k}\right\| \leq\left\|T_{k}-T_{k-1}\right\|+\left\|T_{k-1}\right\|<2 \cdot \varepsilon_{k-1}+\left\|T_{k-1}\right\|<=+\frac{4}{3}
$$

e uma vez que $\varepsilon>0$ é arbitrário, temos $\left\|T_{k}\right\| \leq 4 / 3$, como faltava mostrar. *

Passamos a mostrar duas desigualdades que nos serào úteis na obtenção da desigualdade (3.9).

$$
\left\|T_{k+1}\right\| \geq\left\|T_{k}\right\|+\varepsilon_{k} \cdot\left\|T_{k}\right\|^{2}-4 \varepsilon_{k}^{2} \quad, \quad \forall k \geq 1
$$

De fato, uma vez que $x_{k} \in \mathcal{S}_{X}(\|\cdot\|)$, temos

$$
\begin{aligned}
\left\|T_{k+1}\right\| & \geq\left\|T_{k+1} x_{k}\right\| \stackrel{(3.7)}{=}\left\|\left(T_{k} x_{k}\right)+\varepsilon_{k} \cdot f_{k}\left(T_{k} x_{k}\right) \cdot\left(T_{k} \cdot x_{k}\right)\right\|= \\
& =\left\|T_{k} x_{k}\right\| \cdot\left(1+\varepsilon_{k} \cdot f_{k}\left(T_{k} x_{k}\right)\right) \stackrel{(3.6)}{=}\left\|T_{k} x_{k}\right\| \cdot\left(1+\varepsilon_{k} \cdot\left\|T_{k} x_{k}\right\|\right) \geq \\
& \stackrel{(3.5)}{\geq}\left(\left\|T_{k}\right\|-\varepsilon_{k}^{2}\right) \cdot\left(1+\varepsilon_{k} \cdot\left\|T_{k} x_{k}\right\|\right) \stackrel{(3.5)}{\geq}\left(\left\|T_{k}\right\|-\varepsilon_{k}^{2}\right) \cdot\left(1+\varepsilon_{k} \cdot\left\|T_{k}\right\|-\varepsilon_{k}^{3}\right)
\end{aligned}
$$

então segue que

$$
\begin{aligned}
\left\|T_{k+1}\right\| & \geq\left\|T_{k}\right\|+\varepsilon_{k}\left\|T_{k}\right\|^{2}-\varepsilon_{k}^{3}\left\|T_{k}\right\|-\varepsilon_{k}^{2}-\varepsilon_{k}^{3}\left\|T_{k}\right\|+\varepsilon_{k}^{5} \\
& =\left\|T_{k}\right\|+\varepsilon_{k}\left\|T_{k}\right\|^{2}-2 \varepsilon_{k}^{3}\left\|T_{k}\right\|-\varepsilon_{k}^{2}+\varepsilon_{k}^{5}
\end{aligned}
$$

e, para alcançar o desejado, basta mostrar que

$$
-2 \varepsilon_{k}^{3}\left\|T_{k}\right\|-\varepsilon_{k}^{2}+\varepsilon_{k}^{5} \geq-4 \varepsilon_{k}^{2}
$$

ou, equivalentemente, que

$$
\varepsilon_{k}^{2}\left[3-2 \varepsilon_{k}\left\|T_{k}\right\|+\varepsilon_{k}^{3}\right] \geq 0 .
$$

Para tanto usamos $\left\|T_{k}\right\| \leq 4 / 3$ e $0<\varepsilon_{k}<1 /(10 k)$, como fazemos a seguir. Para todo $k \geq 1$ escrevemos

$$
\begin{aligned}
3-2 \varepsilon_{k}\left\|T_{k}\right\|+\varepsilon_{k}^{3} & \geq 3+2 \varepsilon_{k} \cdot\left(-\frac{4}{3}\right)+\varepsilon_{k}^{3}=3-\frac{8}{3} \varepsilon_{k}+\varepsilon_{k}^{3} \geq 3-3 \varepsilon_{k}+\varepsilon_{k}^{3} \\
& >3+3 \cdot\left(-\frac{1}{10 k}\right)+\varepsilon_{k}^{3}=3-\frac{3}{10 k}+\varepsilon_{k}^{3} \geq 3-\frac{3}{10 k}>0
\end{aligned}
$$


e concluimos a validade da equivalência anterior.

Façamos agora a segunda desigualdade auxiliar:

$$
\left\|T_{k}\right\| \geq\left\|T_{j}\right\| \geq 1, \quad \forall 1 \leq j \leq k-1, \quad \forall k \geq 2 .
$$

Façamos por indução em $k$.

Caso $k=2$ : como pela expressào (3.4) temos $\left\|T_{1}\right\|=\|T\|=1$, segue da expressão (3.10) que

$$
\left\|T_{2}\right\| \geq\left\|T_{1}\right\|+\varepsilon_{1}\left\|T_{1}\right\|^{2}-4 \varepsilon_{1}^{2}
$$

e para mostrarmos que $\left\|T_{2}\right\| \geq\left\|T_{1}\right\|$, basta garantir que

$$
\varepsilon_{1}\left\|T_{1}\right\|^{2}-4 \varepsilon_{1}^{2}=\varepsilon_{1}\left(\left\|T_{1}\right\|^{2}-4 \varepsilon_{1}\right) \geq 0 .
$$

Como $\varepsilon_{1}>0$ e $\left\|T_{1}\right\|^{2}=1$. basta certificar-se que $\varepsilon_{1} \leq 1 / 4$. De fato,

$$
2 \sum_{i \geq 1} \varepsilon_{i}<\varepsilon<\frac{1}{3}
$$

com $\varepsilon_{i}>0$ (para todo $i \geq 1$ ) garante o desejado.

Considere como hipótese de indução que tenhamos,para $k=p>2$

$$
\left\|T_{p}\right\| \geq\left\|T_{p-1}\right\| \geq \cdots \geq\left\|T_{1}\right\| .
$$

Provemos para $k=p+1$ : de (3.10) segue que

$$
\left\|T_{p+1}\right\| \geq\left\|T_{p}\right\|+\varepsilon_{p} \cdot\left\|T_{p}\right\|^{2}-4 \varepsilon_{p}^{2}
$$

e como $\varepsilon_{p}>0$ nos garante

$$
\varepsilon_{p}\left(\left\|T_{p}\right\|^{2}-4 \varepsilon_{p}\right) \geq 0 \Leftrightarrow\left\|T_{p}\right\|^{2} \geq 4 \varepsilon_{p}
$$

precisamos garantir que $\varepsilon_{p} \leq\left\|T_{p}\right\|^{2} / 4$. Como $\varepsilon_{p} \leq 1 / 4$ (pelo mesmo argumento usado em $\varepsilon_{1}$ ) e $\left\|T_{p}\right\|^{2} \geq 1$, então

$$
\frac{\left\|T_{p}\right\|^{2}}{4} \geq \frac{1}{4} \geq \varepsilon_{p}
$$


como esperado.

Passamos à prova da desigualdade (3.9), aqui repetida por conveniência:

$$
\left|f_{j}\left(T_{j} x_{k}\right)\right| \geq\left\|T_{j}\right\|-6 \varepsilon_{j} \quad, \forall 1 \leq j \leq k-1, \forall k \geq 2 .
$$

De fato, como

$$
\left\|T_{j+1} x_{k}\right\| \geq\left\|T_{k} x_{k}\right\|-\left\|\left(T_{k}-T_{j+1}\right)\left(x_{k}\right)\right\| \geq\left\|T_{k} x_{k}\right\|-\left\|T_{k}-T_{j+1}\right\| \cdot\left\|x_{k}\right\|,
$$

segue que para cada $x_{k} \in \mathcal{S}_{X}(\|\cdot\|)$ temos $\left\|T_{j+1} x_{k}\right\| \geq\left\|T_{k} x_{k}\right\|-\left\|T_{k}-T_{j+1}\right\|$.

Valendo-nos de (3.5), (3.8) e (3.11), nesta ordem, para todo $1 \leq j \leq k-2$ tem-se

$$
\left\|T_{j+1} x_{k}\right\| \geq\left\|T_{k}\right\|-\varepsilon_{k}^{2}-2 \sum_{i=j+1}^{k-1} \varepsilon_{i} \geq\left\|T_{j+1}\right\|-\varepsilon_{k}^{2}-2 \sum_{i=j+1}^{k-1} \varepsilon_{i},
$$

para qualquer $k \geq 2$.

Lembrando ainda que a sequência $\left(\varepsilon_{i}\right)_{i \geq 1}$ é decrescente e, além disso, $2 \sum_{i \geq j+1} \varepsilon_{i}<\varepsilon_{j}^{2}$, temos

$$
\left\|T_{j+1} x_{k}\right\| \geq\left\|T_{j+1}\right\|-\varepsilon_{k}^{2}-\varepsilon_{j}^{2}>\left\|T_{j+1}\right\|-2 \varepsilon_{j}^{2},
$$

para todo $1 \leq j \leq k-2$.

Pela expressão (3.7) segue então

$$
\varepsilon_{j} \cdot f_{j}\left(T_{j} x_{k}\right) \cdot\left(T_{j} x_{k}\right)+T_{j} x_{k}=T_{j+1} x_{k} \quad, \forall x \in X
$$

donde garantimos (pela definição de $\left\|T_{j}\right\|$ e da desigualdade recém-obtida para $\left.\left\|T_{j+1} x_{k}\right\|\right)$ que

$$
\varepsilon_{j} \cdot\left|f_{j}\left(T_{j} x_{k}\right)\right| \cdot\left\|T_{j}\right\|+\left\|T_{j}\right\| \geq\left\|T_{j+1} x_{k}\right\| \geq\left\|T_{j+1}\right\|-2 \varepsilon_{j}^{2} .
$$

Como por (3.10) temos $\left\|T_{j+1}\right\| \geq\left\|T_{j}\right\|+\varepsilon_{j} \cdot\left\|T_{j}\right\|^{2}-4 \varepsilon_{j}^{2}$, então

$$
\varepsilon_{j}\left|f_{j}\left(T_{j} x_{k}\right)\right| \cdot\left\|T_{j}\right\|+\left\|T_{j}\right\| \geq\left\|T_{j}\right\|+\varepsilon_{j} \cdot\left\|T_{j}\right\|^{2}-6 \varepsilon_{j}^{2}
$$


e, efetuando-se os cancelamentos óbvios, teremos

$$
\left|f_{j}\left(T_{j} x_{k}\right)\right| \cdot\left\|T_{j}\right\| \geq\left\|T_{j}\right\|^{2}-6 \varepsilon_{j} .
$$

Uma vez que $1 \leq\left\|T_{j}\right\| \leq 4 / 3$, segue de imediato

$$
\left|f_{j}\left(T_{j} x_{k}\right)\right| \geq\left\|T_{j}\right\|-\frac{6 \varepsilon_{j}}{\left\|T_{j}\right\|} \geq\left\|T_{j}\right\|-6 \varepsilon_{j} \quad, \forall 1 \leq j \leq k-2, \quad \forall k \geq 2,
$$

o que queríamos mostrar.

\section{Teorema 3.6}

Sejam $X$ e $Y$ dois espaços de Banach. Entâo $\mathcal{P}_{0}(X, Y)$ é denso em $\mathcal{L}(X, Y)$.

Prova: Considere as sequências e respectivas propriedades envolvidas no lema (3.5). A desigualdade (3.8) nele presente, bem como as desigualdades associadas à sequência $\left(\varepsilon_{i}\right)_{i \geq 1}$, nos permitem concluir que $\left(T_{k}\right)_{k \geq 1}$ constitui uma sequência de Cauchy em $\mathcal{L}(X, Y)$. Sendo este último espaço completo (pelo teorema (1.8)), segue a existência de $\hat{T} \in \mathcal{L}(X, Y)$ tal que $\lim _{k \rightarrow \infty} T_{k}=\hat{T}$.

A continuidade da norma nos permite afirmar ${ }^{3}$ que

$$
\left\|\hat{T}-T_{j}\right\| \leq 2 \sum_{i \geq j} \varepsilon_{i}<\varepsilon_{j-1}^{2} \quad, \forall j \geq 2 .
$$

Passemos agora a verificar que $\hat{T} \in \mathcal{P}_{0}(X, Y)$ :

De fato, observando que no lema (3.5) criamos $\left(x_{k}\right)_{k \geq 1} \subset X^{r}$ e $\left(f_{k}\right)_{k \geq 1} \subset$ $Y^{\prime}$ satisfazendo $\left\|x_{k}\right\|=\left\|f_{k}\right\|=1$ para todo $k \geq 1$, sendo $X$ e $Y$ espaços de Banach e $\hat{T} \in \mathcal{L}(X, Y)$, será suficiente garantirmos que

$$
\left|f_{j}\left(\hat{T} x_{k}\right)\right| \geq\|\hat{T}\|-\frac{1}{j} \quad, \forall 2 \leq j \leq k-1, \quad \forall k \geq 3 .
$$

Verifiquemos a desigualdade desejada.

$$
\begin{aligned}
\left|f_{j}\left(\hat{T} x_{k}\right)\right| & \geq\left|f_{j}\left(T_{j} x_{k}\right)\right|-\left|f_{j}\left(T_{j} x_{k}\right)-f_{j}\left(\hat{T} x_{k}\right)\right|= \\
& =\left|f_{j}\left(T_{j} x_{k}\right)\right|-\left|f_{j}\left(\left(T_{j}-\hat{T}\right)\left(x_{k}\right)\right)\right| \geq\left|f_{j}\left(T_{j} x_{k}\right)\right|-\left\|f_{j}\right\| \cdot\left\|T_{j}-\hat{T}\right\|
\end{aligned}
$$

\footnotetext{
${ }^{3}$ novamente usando $(3.8)$ e $(3.3)$
} 
e valendo-nos de $\left\|f_{j}\right\|=1$ e das expressões (3.9) e (3.12) temos, para qualquer $2 \leq j \leq k-1 \quad(k \geq 3)$, que

$$
\left|f_{j}\left(\hat{T} x_{k}\right)\right| \geq\left\|T_{j}\right\|-6 \cdot \varepsilon_{j}-\varepsilon_{j-1}^{2} .
$$

Como pela desigualdade triangular, (3.8) e (3.3) temos

$$
\begin{aligned}
\left\|T_{k}\right\| & \leq\left\|T_{k}-T_{j}\right\|+\left\|T_{j}\right\| \leq 2 \sum_{i=j}^{k-1} \varepsilon_{i}+\left\|T_{j}\right\|<2 \sum_{i \geq j} \varepsilon_{i}+\left\|T_{j}\right\|< \\
& <\varepsilon_{j-1}^{2}+\left\|T_{j}\right\|,
\end{aligned}
$$

então fazendo $k \rightarrow \infty$ podemos afirmar, a partir da continuidade da norma, que $\|\hat{T}\| \leq \varepsilon_{j-1}^{2}+\left\|T_{j}\right\|$ e, portanto, $\left\|T_{j}\right\|-\varepsilon_{j-1}^{2} \geq\|\hat{T}\|-2 \varepsilon_{j-1}^{2}$.

Fazendo a substituição na expressão anterior teremos, para $2 \leq j \leq k-1$,

$$
\begin{aligned}
\left|f_{j}\left(\hat{T} x_{k}\right)\right| & \geq\left\|T_{j}\right\|-6 \varepsilon_{j}-\varepsilon_{j-1}^{2} \geq\|\hat{T}\|-6 \varepsilon_{j}-2 \varepsilon_{j-1}^{2} \geq \\
& \geq\|\hat{T}\|-\frac{6}{10 j}-2 \varepsilon_{j-1}^{2} .
\end{aligned}
$$

Como $j \leq 20(j-1)^{2}$ para todo $j \geq 2$ nos garante que

$$
-\frac{6}{10 \cdot j}-\frac{2}{10^{2} \cdot(j-1)^{2}} \geq-\frac{1}{j}
$$

e para $j$ nestas condições $\varepsilon_{j-1} \leq 1 /(10 \cdot(j-1))$ implica em

$$
-\frac{6}{10 \cdot j}-2 \cdot \varepsilon_{j-1}^{2} \geq-\frac{6}{10 \cdot j}-\frac{2}{10^{2} \cdot(j-1)^{2}},
$$

podemos concluir para todo $j \geq 2$ que

$$
\|\hat{T}\|-\frac{6}{10 j}-2 \varepsilon_{j-1}^{2} \geq\|\hat{T}\|-\frac{1}{j} .
$$

Segue que $\left|f_{j}\left(\hat{T} x_{k}\right)\right| \geq\|\hat{T}\|-1 / j$ e o lema (3.3) nos garante então $\hat{T} \in \mathcal{P}_{0}(X, Y)$ como desejado.

Portanto, dados quaisquer $T \in \mathcal{L}(X, Y)$ e $0<\varepsilon<1 / 3$, existe $\hat{T} \in \mathcal{P}_{0}(X, Y)$ tal que $\|\hat{T}-T\| \leq \varepsilon$; o teorema está provado. 


\section{Corolário 3.7}

Um espaço de Banach $\left(X^{\prime},\|\cdot\|\right)$ é reflexivo se e só se $\mathcal{N} \mathcal{A}\left(X^{\prime}, Y\right)$ é denso em $\mathcal{L}(X, Y)$ para qualquer espaço de Banach $(Y,\|\cdot\|)$.

Prova: Mostremos inicialmente que $\mathcal{N} \mathcal{A}(X, Y) \subset \mathcal{P}_{0}(X, Y)$ :

Fixado $T \in \mathcal{N} \mathcal{A}(X, Y)$, tome $x_{0} \in \mathcal{B}_{X}(\|\cdot\|)$ tal que $\left\|T x_{0}\right\|=\|T\|$. Como $\left\|J\left(x_{0}\right)\right\|=\left\|x_{0}\right\| \leq 1$, então $J\left(x_{0}\right) \in \mathcal{B}_{X^{\prime \prime}}(\|\cdot\|)$.

Pelo teorema (1.28) temos, para todo $x \in X$, que

$$
\left\|T^{\prime \prime}\left(J\left(x_{0}\right)\right)\right\|=\left\|J\left(T x_{0}\right)\right\|=\left\|T x_{0}\right\|=\|T\|=\left\|T^{\prime \prime}\right\|
$$

e, portanto, $T \in \mathcal{P}_{0}(X, Y)$ como desejado.

Admitindo por hipótese $X$ reflexivo, qualquer ponto da bola $\mathcal{B}_{X^{\prime \prime}}(\|\cdot\|)$ é imagem, pela imersão canônica $J$, de algum ponto da bola $\mathcal{B}_{X}(\|\cdot\|)$. Assim sendo, dado $\xi^{\prime \prime} \in \mathcal{B}_{X^{\prime \prime}}(\|\cdot\|)$ tal que $\left\|T^{\prime \prime}\right\|=\left\|T^{\prime \prime} \xi^{\prime \prime}\right\|$, existe $x_{0} \in \mathcal{B}_{X}(\|\cdot\|)$ tal que $\xi^{\prime \prime}=J\left(x_{0}\right)$ e então teremos

$$
\|T\|=\left\|T^{\prime \prime}\right\|=\left\|T^{\prime \prime} \xi^{\prime \prime}\right\|=\left\|T^{\prime \prime}\left(J\left(x_{0}\right)\right)\right\|=\left\|T x_{0}\right\|,
$$

o que garante então $\mathcal{P}_{0}(X, Y) \subset \mathcal{N} \mathcal{A}(X, Y)$. Tem-se $\mathcal{N} \mathcal{A}(X, Y)=\mathcal{P}_{0}(X, Y)$ e o teorema anterior assegura o desejado.

A recíproca segue do teorema $(2.1)$ para $(Y,\|\cdot\|)=(\mathbb{R},|\cdot|)$.

\section{Observação 3.8}

É interessante comentar que, com argumentos similares aos utilizados no corolário (3.7), pode-se facilmente mostrar que

$$
T \in \mathcal{N} \mathcal{A}(X, Y) \Rightarrow T^{\prime} \in \mathcal{N} \mathcal{A}\left(Y^{\prime}, X^{\prime}\right) \Rightarrow T^{\prime \prime} \in \mathcal{N} \mathcal{A}\left(X^{\prime \prime}, Y^{\prime \prime}\right) .
$$

Em 1973 V. Zizler ([Zizler]) publicou um artigo no qual, valendo-se do mesmo tipo de técnica usado por Lindenstrauss, melhora o resultado provando que o subespaço constituido pelos operadores $T \in \mathcal{L}(X, Y)$ tais que $T^{\prime}$ atinge sua norma é denso em $\mathcal{L}(X, Y)$. 


\subsection{Espaços estritamente e uniformemente con- vexos}

As definições e propriedades que apresentaremos nesta seção estão intimamente relacionadas com os resultados (obtidos por J.Lindenstrauss) associados à propriedade $A$, como veremos no decorrer da próxima seção.

\section{Definição 3.9 (espaços estritamente convexos)}

Dizemos que um espaço normado $(X,\|\cdot\|)$ é estritamente convero se dados quaisquer $\stackrel{2}{\sim}$ pontos distintos de sua esfera, o segmento que os une nâo tem quaisquer outros pontos à ela pertencentes, i.e, dados quaisquer $x, y \in \mathcal{S}_{X}(\|\cdot\|)$ distintos,

$$
\|(1-t) x+t y\|<1 \quad, \quad \forall t \in(0,1) .
$$

Proposição 3.10 No espaço normado $(X,\|\cdot\|)$ são equivalentes:

a) $(X,\|\cdot\|)$ é estritamente convexo,

b) Para cada $f \in X^{\prime}$ não nulo, existe no máximo um ponto da esfera $\mathcal{S}_{X}(\|\cdot\|)$ no qual o funcional atinge sua norma,

c) $S \in x, y \in \mathcal{S}_{X}(\|\cdot\|)$ são distintos entẫo $\|(x+y) / 2\|<1$,

d) Se $x, y \in \mathcal{S}_{X}(\|\cdot\|)$ são tais que $\|x+y\|=2$, então $x=y$.

e) Se $\|x+y\|=\|x\|+\|y\| \in x \neq 0$, então existe $\lambda \geq 0$ tal que $y=\lambda x$.

Prova: Faremos a demonstração pelo seguinte roteiro :

- $a) \Rightarrow b) \Rightarrow e(\Rightarrow a) \quad ; \bullet c) \Leftrightarrow d) \quad ; \quad a) \Rightarrow d) \quad ; \quad c) \Rightarrow e)$.

Temos de fato :

- $a) \Rightarrow b)$ : Admita existir $f \in X^{\prime}, f \neq 0 \quad($ daí $\|f\|>0$ ) e elementos $x, y \in \mathcal{S}_{X}(\|\cdot\|)$ tais que $f(x)=f(y)=\|f\|$. Sem perda de generalidade podemos assumir $\|f\|=1$ e teremos

$$
f((1-t) x+t y)=(1-t) f(x)+t f(y)=\|f\|=1 \quad, \forall t \in(0,1) .
$$


Logo

$$
\begin{aligned}
1 & =|f((1-t) x+t y)| \leq\|f\| \cdot\|(1-t) x+t y\|= \\
& =\|(1-t) x+t y\| \leq(1-t)\|x\|+t\|y\|=1
\end{aligned}
$$

e então $\|(1-t) x+t y\|=1, \quad \forall t \in(0,1)$. Sendo $(X,\|\cdot\|)$ estritamente convexo, não existem elementos distintos de $\mathcal{S}_{X}(\|\cdot\|)$ nestas condições e, portanto, $x=y$ como esperado.

$b) \Rightarrow \epsilon)$ : Suponha $x, y \in X, x \neq 0$ e $\|x+y\|=\|x\|+\|y\|$.

Pelo corolário (1.17), existe $f \in \mathcal{S}_{X^{\prime}}(\|\cdot\|)$ tal que $f(x+y)=\|(x+y)\|$ e, portanto,

$$
\begin{aligned}
\|f\|\|x\|+\|f\|\|y\| & =\|x\|+\|y\|=\|x+y\|=|f(x+y)|= \\
& =|f(x)+f(y)| \leq|f(x)|+|f(y)| \leq \\
& \leq\|f\|\|x\|+\|f\|\|y\|
\end{aligned}
$$

donde segue que

$$
|f(x)|+|f(y)|=\|f\|\|x\|+\|f\|\|y\| .
$$

Como $|f(x)| \leq\|f\|\|x\|$ e $|f(y)| \leq\|f\|\|y\|$, segue da igualdade anterior que $|f(x)|=\|f\|\|x\|$ e $|f(y)|=\|f\|\|y\|$. De $x \neq 0$, caso $y=0$ teríamos $\lambda=0$ encerrando o resultado; do contrário $x \neq 0$ e $y \neq 0$ nos permitem escrever, a partir das igualdades acima, que

$$
\left|f\left(\frac{x}{\|x\|}\right)\right|=\left|f\left(\frac{y}{\|y\|}\right)\right|=\|f\|=1
$$

e, pela hipótese, garantimos $x /\|x\|=y /\|y\|$. Note que $\lambda=\|y\| /\|x\|$ é o número real procurado. 
$e) \Rightarrow a)$ : Sejam $x, y$ em $\mathcal{S}_{X}(\|\cdot\|)$ e tais que $\left\|\left(1-t_{0}\right) x+t_{0} y\right\|=1$ para algum $t_{0} \in(0,1)$.

Trivialmente

$$
\left\|\left(1-t_{0}\right) x\right\|+\left\|t_{0} y\right\|=1=\left\|\left(1-t_{0}\right) x+t_{0} y\right\|
$$

e como $t_{0} y \neq 0$, deve existir $\lambda \geq 0$ tal que

$$
\left(1-t_{0}\right) x=\lambda t_{0} y .
$$

De $\left(1-t_{0}\right) \cdot x \neq 0$, segue que $\lambda>0$ e portanto, de

$$
1-t_{0}=\left\|\left(1-t_{0}\right) x\right\|=\left\|\lambda t_{0} y\right\|=\lambda t_{0}
$$

temos, por (3.13), que $\lambda t_{0} x=\lambda t_{0} y$. Como $\lambda t_{0} \neq 0$ temos $x=y$.

- $c) \Leftrightarrow d)$ :

$(\Rightarrow)$ : Considere $x, y \in \mathcal{S}_{X}(\|\cdot\|)$ tais que $\|x+y\|=2$. Da hipótese segue que tais elementos não podem ser distintos pois se o fossem teríamos $\|x+y\|<2$.

$(\Leftarrow)$ : Para quaisquer $x, y \in \mathcal{S}_{X}(\|\cdot\|)$ distintos temos $\|x+y\|<2$ pois, do contrário, $\|x+y\|=2$ implicaria em $x=y$.

- $a) \Rightarrow d):$ Sejam $x, y \in \mathcal{S}_{X}(\|\cdot\|)$ tais que $\|x+y\|=2$. Temos então

$$
\|1 / 2 x+1 / 2 y\|=1
$$

e pela estrita convexidade segue que $x=y$, como desejado.

- $c) \Rightarrow e)$ : Observe inicialmente que para quaisquer $x, y \in X$ temos:

$$
\begin{aligned}
(\|x\|+\|y\|) \cdot\|x+y\| & =\|(\|x\|+\|y\|) \cdot(x+y)\|= \\
& =\|\| x\|\cdot x+\| y\|\cdot y+\| x\|\cdot y+\| y\|\cdot x\| \leq \\
& \leq\|x\|^{2}+\|y\|^{2}+\|\| x\|\cdot y+\| y\|\cdot x\| .
\end{aligned}
$$


Fazendo pela contra-recíproca, admitimos a existência de $x, y$ em $X$ linearmente independentes e tais que $\|x\|+\|y\|=\|x+y\|$. Tomando como elementos distintos da esfera os vetores $z=x /\|x\|$ e $w=y /\|y\|$, temos $\|z+w\| \leq 2$. Em realidade $\|z+w\|=2$ pois caso $\|z+w\|<2$ teríamos

$$
\left\|\frac{x \cdot\|y\|+y \cdot\|x\|}{\|x\| \cdot\|y\|}\right\|=\left\|\frac{x}{\|x\|}+\frac{y}{\|y\|}\right\|=\|z+w\|<2
$$

donde poderíamos concluir que

$$
\|x \cdot\| y\|+y \cdot\| x\|\|<2\|x\| \cdot\|y\| .
$$

Das primeiras desigualdades obtidas neste item seguiria que

$$
(\|x\|+\|y\|) \cdot\|x+y\|<\|x\|^{2}+\|y\|^{2}+2\|x\| \cdot\|y\|=(\|x\|+\|y\|)^{2},
$$

donde teríamos $\|x+y\|<\|x\|+\|y\|$, absurdo.

Uma vez que obtivemos dois pontos distintos da esfera $z, w$ tais que $\|z+w\|=2$, negamos a hipótese como era desejado.

\section{Observação 3.11}

Utilizaremos mais adiante uma afirmação trivialmente equivalente à apresentada no item d) da proposição anterior, a saber :

Sejam $a>0$ e $x, y \in a \cdot \mathcal{S}_{X}(\|\cdot\|)=\left\{x \in X^{\prime}:\|x\|=a\right\}$. Se $\|x+y\|=2 a$, então $x=y$.

\section{Exemplo 3.12}

Os espaços $\left(l^{p},\|\cdot\|\right)$ e $\left(L^{p}([0,1]),\|\cdot\|\right)$ (normas usuais) são estritamente convexos para qualquer $1<p<\infty$. Os espaços $\left(c_{0},\|\cdot\|\right),\left(l^{1},\|\cdot\|\right) e$ $\left(L^{1}([0,1]),\|\cdot\|\right)$ (normas usuais) não têm tal propriedade.

Mencionamos estes fatos neste momento mas postergamos as justificativas até o exemplo (3.18) e corolário (3.30). 


\section{Observação $\mathbf{3 . 1 3}$}

O teorema do suporte (corolário (1.15)) nos garante que, num espaço normado $(X,\|\cdot\|)$, todo ponto da esfera unitária $\mathcal{S}_{X}(\|\cdot\|)$ admite um hiperplanosuporte para a bola unitária $\mathcal{B}_{X}(\|\cdot\|)$. Neste sentido, a caracterização b) da proposiçào (3.10) nos ensina que, em espaços normados estritamente convexos, os hiperplanos-suporte para $\mathcal{B}_{X}(\|\cdot\|)$ associados a diferentes pontos da esfera são sempre distintos. Vejamos o porquê:

Considere $\xi, \eta \in \mathcal{S}_{X}(\|\cdot\|)$ distintos e tais que admitam o mesmo hiperplanosuporte $\mathcal{H}(f, a)$ para a bola $\mathcal{B}_{X}(\|\cdot\|)$.

Podemos admitir sem perda de generalidade que

$$
f(\xi)=\alpha \quad \text { e } \sup _{x \in \mathcal{B}_{X}(\|\cdot\|)}|f(x)| \leq \alpha,
$$

uma vez que do contrário o funcional $g=-f$ (com o real $\beta=-\alpha$ ) serviria aos nossos propósitos. De $f(\eta)=\alpha=f(\xi), f$ assumiria seu valor máximo em pelo menos dois pontos distintos da esfera, o que contradiz a estrita convexidade do espaço.

\section{Definição 3.14 (espaço uniformemente convexo)}

Dizemos que um espaço normado $(X,\|\cdot\|)$ é uniformemente convexo se dado qualquer $\varepsilon>0$, existe um $\delta>0$ tal que

$$
\left\|\frac{x+y}{2}\right\| \leq 1-\delta
$$

para quaisquer $x, y \in \mathcal{S}_{X}(\|\cdot\|)$ tais que $\|x-y\| \geq \approx$ (logo somente $0<\varepsilon \leq 2$ é de interesse).

\section{Observação 3.15}

Perceba que num espaço de Banach uniformemente convexo, dado $\varepsilon>0$ e quaisquer dois pontos da esfera que distam entre si pelo menos $\varepsilon$, o ponto médio do segmento que os une não apenas se encontra no interior da bola unitária ${ }^{4}$; o faz distando pelo menos um $\delta=\delta(\varepsilon)>0$ da esfera unitária.

\footnotetext{
${ }^{4}$ o que caracteriza os espaços estritamente convexos.
} 


\section{Exemplo 3.16}

Vejamos dois eremplos interessantes que ressaltam o fato da uniformidade convexa estar diretamente associada à norma do espaço de Banach em questão.

Mostremos que o $\mathbb{R}^{n}$ é uniformemente convexo quando munido da norma euclidiana, mas não o é quando associamos a ele a norma $\|\cdot\|_{1}$.

- $\left(\mathbb{R}^{n},\|\cdot\|\right)$ é uniformemente convexo para a norma euclidiana.

De fato, tome quaisquer $x=\left(x_{1}, x_{2}, \ldots, x_{n}\right)$ e $y=\left(y_{1}, y_{2}, \ldots, y_{n}\right)$ elementos de $\mathcal{S}_{\mathbb{R}^{n}}(\|\cdot\|)$. Temos então

$$
\begin{aligned}
\left\|\frac{x+y}{2}\right\|^{2}+\left\|\frac{x-y}{2}\right\|^{2} & =\frac{1}{4}\left[\sum_{i=1}^{n}\left(x_{i}+y_{i}\right)^{2}+\sum_{i=1}^{n}\left(x_{i}-y_{i}\right)^{2}\right]= \\
& =\frac{1}{4} \sum_{i=1}^{n}\left(2 x_{i}^{2}+2 y_{i}^{2}\right)=\frac{1}{2}\left(\|x\|^{2}+\|y\|^{2}\right) .
\end{aligned}
$$

Dado $\varepsilon>0$, tome $0<\delta<1$ tal que $(1-\delta)^{2}=1-\varepsilon^{2} / 4$. Pela expressão acima sabemos que, escolhendo quaisquer $x, y \in \mathcal{S}_{\mathbb{R}^{n}}(\|\cdot\|)$ tais que $\|x-y\| \geq \varepsilon$, tem-se

$$
\left\|\frac{x+y}{2}\right\|^{2}=\frac{1}{2}\left(\|x\|^{2}+\|y\|^{2}\right)-\left\|\frac{x-y}{2}\right\|^{2}=1-\left\|\frac{x-y}{2}\right\|^{2} \leq(1-\delta)^{2}
$$

e concluimos o desejado.

- $\left(\mathbb{R}^{n},\|\cdot\|_{1}\right)$ não é uniformemente convexo.

De fato, tome em particular $x=(1,0,0,0, \ldots)$ e $y=(0,1,0,0, \ldots)$.

Uma vez que $x, y \in \mathcal{S}_{\mathbb{R}^{n}}\left(\|\cdot\|_{1}\right)$ são tais que

$$
\|x-y\|_{1}=\sum_{i=1}^{n}\left|x_{i}-y_{i}\right|=2
$$

tomando $\varepsilon=2>0$ teremos

$$
\left\|\frac{x+y}{2}\right\|_{1}=1>1-\delta
$$

para qualquer $\delta>0$ fixado. 


\section{Observação 3.17}

Uma ligeira modificação no primeiro item do exemplo (3.16) acaba por provar que todo espaço $H$ munido de produto interno é uniformemente convexo.

De fato, é imediato constatar para um tal espaço $H$ a validade da chamada "igualdade do paralelogramo" :

$$
\|x+y\|^{2}+\|x-y\|^{2}=2\left(\|x\|^{2}+\|y\|^{2}\right), \forall x, y \in H
$$

e portanto temos facilmente

$$
\left\|\frac{x+y}{2}\right\|^{2}+\left\|\frac{x-y}{2}\right\|^{2}=\frac{1}{2}\left(\|x\|^{2}+\|y\|^{2}\right)
$$

e procede-se exatamente como no exemplo mencionado.

É interessante notar que o exemplo seguinte também culmina na mesma expressão.

Exemplo 3.18 Os espaços $\left(l^{p},\|\cdot\|\right)$ e $\left(L^{p}([0,1]),\|\cdot\|\right)$ (normas usuais) sâo uniformemente convexos para qualquer $1<p<\infty$.

De fato, façamos para o espaço $\left(l^{p},\|\cdot\|\right) \operatorname{com} 2 \leq p<\infty$ fixado. Valendo-nos da desigualdade

$$
|z+w|^{p}+|z-w|^{p} \leq 2^{p-1}\left(|z|^{p}+|w|^{p}\right)
$$

válida $^{5}$ para quaisquer reais $z \cdot w$ (e $\left.2 \leq p<\infty\right)$, consideremos quaisquer dois elementos $x=\left(x_{i}\right)_{i \geq 1}$ e $y=\left(y_{i}\right)_{i \geq 1}$ de $\mathcal{S}_{l p}(\|\cdot\|)$.

Observando que para cada $i \geq 1$ podemos escrever

$$
\left|x_{i}+y_{i}\right|^{p}+\left|x_{i}-y_{i}\right|^{p} \leq 2^{p-1}\left(\left|x_{i}\right|^{p}+\left|y_{i}\right|^{p}\right), \forall i \geq 1,
$$

para todo $n \geq 1$ temos portanto

$$
\sum_{i=1}^{n}\left|x_{i}+y_{i}\right|^{p}+\sum_{i=1}^{n}\left|x_{i}-y_{i}\right|^{p} \leq 2^{p-1}\left(\sum_{i=1}^{n}\left|x_{i}\right|^{p}+\sum_{i=1}^{n}\left|y_{i}\right|^{p}\right) .
$$

\footnotetext{
${ }^{5}$ Vide [Köthe], 26.7 (3), 355.
} 
Da convergência das séries presentes segue que

$$
\|x+y\|^{p}+\|x-y\|^{p} \leq 2^{p-1}\left(\|x\|^{p}+\|y\|^{p}\right), \forall p \geq 2 .
$$

Uma vez que

$$
\left\|\frac{x+y}{2}\right\|^{p}+\left\|\frac{x-y}{2}\right\|^{p} \leq \frac{1}{2}\left(\|x\|^{p}+\|y\|^{p}\right)
$$

encerramos procedendo exatamente como já mostrado.

Os casos associados a $1<p<2$, bem como os envolvidos nos $L^{p}$ s não são tão imediatos e sugerimos [Köthe], 26.7 (12), 358.

\section{Proposição 3.19 (Milman-Pettis)}

Todo espaço de Banach $(X,\|\cdot\|)$ uniformemente convexo é reflexivo.

\section{Prova:}

Considere sem perda de generalidade $f \in \mathcal{S}_{X^{\prime}}(\|\cdot\|)$ e uma sequência $\left(x_{n}\right)_{n \geq 1} \subset \mathcal{S}_{X}(\|\cdot\|)$ tal que $f\left(x_{n}\right) \stackrel{n \rightarrow \infty}{\longrightarrow}\|f\|=1$.

Admita momentaneamente que tal $\left(x_{n}\right)_{n \geq 1}$ seja sequência de Cauchy em $X$; então converge para digamos $x_{0} \in X$. A continuidade da norma garante que $x_{0} \in \mathcal{S}_{X}(\|\cdot\|)$ e a continuidade do funcional implica em $f\left(x_{0}\right)=\|f\|$, assegurando que $f$ atinge sua norma. Pelo teorema (2.1) temos $X$ reflexivo.

Verifiquemos o postergado: suponha por absurdo que $\left(x_{n}\right)_{n \geq 1}$ não seja Cauchy. Neste caso existem $\varepsilon>0$ e subsequência $\left(x_{n_{k}}\right)_{k \geq 1}$ tais que $\left\|x_{n_{j}}-x_{n_{p}}\right\| \geq \varepsilon$ para quaisquer elementos distintos da subsequência. Uma vez que $\left(x_{n_{k}}\right)_{k \geq 1} \subset \mathcal{S}_{X}(\|\cdot\|)$ e $X$ é uniformemente convexo, garante-se a existência de $\delta>0$ para o qual

$$
\left\|\frac{x_{n_{j}}+x_{n_{p}}}{2}\right\| \leq 1-\delta
$$

e como $|f(x)| \leq\|f\|\|x\|=\|x\|$ para todo $x \in X$, tem-se

$$
\left|f\left(\frac{x_{n_{\jmath}}+x_{n_{p}}}{2}\right)\right| \leq 1-\delta,
$$


o que não pode ocorrer. De fato, como $f\left(x_{n}\right) \stackrel{n \rightarrow \infty}{\longrightarrow} 1$, temos que $f\left(x_{n_{j}}\right) \stackrel{j \rightarrow \infty}{\longrightarrow} 1$ e $f\left(x_{n_{p}}\right) \stackrel{p \rightarrow \infty}{\longrightarrow} 1$. Da linearidade e continuidade

$$
\left|f\left(\frac{x_{n_{\jmath}}+x_{n_{p}}}{2}\right)\right| \stackrel{j, p \rightarrow \infty}{\longrightarrow} 1 .
$$

Definição 3.20 (espaços localmente uniformemente convexos)

Dizemos que um espaço normado $(X,\|\cdot\|)$ é localmente uniformemente convexo em $x_{0} \in \mathcal{S}_{X}(\|\cdot\|)$ se, dado qualquer $\varepsilon>0$, existe $\delta>0$ tal que

$$
\left\|\frac{x_{0}+y}{2}\right\| \leq 1-\delta
$$

para todo $y \in \mathcal{S}_{X}(\|\cdot\|)$ tal que $\left\|x_{0}-y\right\| \geq \varepsilon$ (nestas condiçôes diz-se que a norma é localmente uniformemente convexa em $x_{0}$ ).

Caso $(X,\|\cdot\|)$ seja localmente uniformemente convexo em todo ponto de $\mathcal{S}_{X}(\|\cdot\|)$, diremos que o espaço $(X,\|\cdot\|)$ é localmente uniformemente convexo (o mesmo em relação à norma $\|\cdot\|$ ).

\section{Observação 3.21}

A caracterização de espaços normados estritamente convexos dada pela proposição $(3.10$. c)) nos garante de imediato que todo espaço normado localmente uniformemente convexo é estritamente convexo. Uma vez que (evidentemente) todo espaço uniformemente convexo é localmente uniformemente convexo, segue também que todo espaço uniformemente convexo é estritamente convexo.

Passemos a um lema que nos permitirá caracterizar os espaços localmente uniformemente convexos de maneira similar à apresentada nos estritamente convexos. 


\section{Lema 3.22}

Seja $(X,\|\cdot\|)$ um espaço de Banach. Entioo $(X,\|\cdot\|)$ é localmente uniformemente convexo $\mathrm{em} x_{0} \in \mathcal{S}_{X}(\|\cdot\|)$ se e só se o módulo de convexidade local $\zeta\left(x_{0}, \varepsilon\right)$ definido por

$$
\zeta\left(x_{0}, \varepsilon\right)=\frac{1}{2} \inf _{z \in \Omega\left(\varepsilon, x_{0}\right)}\left\{2-\left\|x_{0}+z\right\|\right\}
$$

onde $\Omega\left(\varepsilon, x_{0}\right)=\left\{z \in \mathcal{S}_{X}(\|\cdot\|),\left\|x_{0}-z\right\| \geq \varepsilon\right\}$ é estritamente positivo para todo $0<\varepsilon \leq 2$.

Prova: Suponha inicialmente que $(X,\|\cdot\|)$ seja localmente uniformemente convexo em $x_{0} \in \mathcal{S}_{X}(\|\cdot\|)$. Dado $0<\varepsilon \leq 2$, existe portanto $\delta=\delta\left(x_{0}, \varepsilon\right)>0$ tal que

$$
2-\left\|x_{0}+y\right\| \geq 2-2(1-\delta)=2 \delta>0
$$

sempre que $y \in \mathcal{S}_{X}(\|\cdot\|)$ é tal que $\left\|x_{0}-y\right\| \geq \varepsilon$. Entào $\zeta\left(x_{0}, \varepsilon\right)>0$.

Reciprocamente, mostremos que se $\zeta\left(x_{0}, \varepsilon\right)>0$ para $x_{0} \in \mathcal{S}_{X}(\|\cdot\|)$ então o espaço de Banach $(X,\|\cdot\|)$ é localmente uniformemente convexo neste ponto.

Fixado $0<\varepsilon_{0} \leq 2$, suponhamos por absurdo que, para cada $n \geq 1$, exista

$$
y_{n} \in \mathcal{S}_{X}(\|\cdot\|) \quad \text { com } \quad\left\|x_{0}-y_{n}\right\| \geq \varepsilon_{0} \quad \text { tal que } \quad\left\|\frac{x_{0}+y_{n}}{2}\right\| \geq 1-\frac{1}{n} .
$$

Construimos assim uma sequência $\left(y_{n}\right)_{n \geq 1} \subset \mathcal{S}_{X}(\|\cdot\|)$ tal que

$$
\left\|x_{0}-y_{n}\right\| \geq \varepsilon_{0} \quad \text { e } 2-\left\|x_{0}+y_{n}\right\| \leq \frac{2}{n}, \forall n \geq 1 \text {. }
$$

As primeiras duas condições de $\left(y_{n}\right)_{n \geq 1}$ garantem que $\left(y_{n}\right)_{n \geq 1} \subset \Omega\left(\varepsilon_{0}, x_{0}\right)$ e, pela terceira, podemos concluir que

$$
\inf _{y \in \Omega\left(\varepsilon_{0}, x_{0}\right)} 2-\left\|x_{0}+y\right\|=0
$$

e portanto $\zeta\left(x_{0}, \varepsilon_{0}\right)=0$, contradição. 


\section{Proposição 3.23}

Considere $(X,\|\cdot\|)$ um espaço de Banach e $x_{0} \in \mathcal{S}_{X}(\|\cdot\|)$ fixado. Sâo equivalentes:

a) A norma $\|\cdot\|$ é localmente uniformemente convexa em $x_{0}$,

b) Se $\left(x_{n}\right)_{n \geq 1} \subset \mathcal{S}_{X}(\|\cdot\|) \in\left\|x_{0}+x_{n}\right\| \stackrel{n \rightarrow \infty}{\longrightarrow} 2$. entâo $\left\|x_{0}-x_{n}\right\| \stackrel{n \rightarrow \infty}{\longrightarrow} 0$,

c) Se $\left(x_{n}\right)_{n \geq 1} \subset X$ étal que $2\left(\left\|x_{0}\right\|^{2}+\left\|x_{n}\right\|^{2}\right)-\left\|x_{0}+x_{n}\right\|^{2} \stackrel{n \rightarrow \infty}{\longrightarrow} 0$ então $\left\|x_{0}-x_{n}\right\| \stackrel{n \rightarrow \infty}{\longrightarrow} 0$,

d) Se $\left(x_{n}\right)_{n \geq 1} \subset X \quad \dot{e}$ tal que $\left\|x_{n}\right\| \stackrel{n \rightarrow \infty}{\longrightarrow}\left\|x_{0}\right\|=1 e$ $\left\|x_{0}+x_{n}\right\| \stackrel{n \rightarrow \infty}{\longrightarrow} 2\left\|x_{0}\right\|=2$ entâo $\left\|x_{0}-x_{n}\right\| \stackrel{n \rightarrow \infty}{\longrightarrow} 0$.

Prova: Faremos a demonstração pelo seguinte roteiro :

- $a) \Leftrightarrow b) \quad ; \quad \bullet c) \Leftrightarrow d$ ) $\mathrm{e} \quad \bullet b) \Leftrightarrow d$ ) .

Temos de fato :

- $a) \Rightarrow b)$ : Suponhamos por absurdo a existência de $\left(x_{n}\right)_{n \geq 1} \subset \mathcal{S}_{X}(\|\cdot\|)$ tal que $\left(2-\left\|x_{0}+x_{n}\right\|\right) \stackrel{n \rightarrow \infty}{\longrightarrow} 0$ mas $\left\|x_{0}-x_{n}\right\| \stackrel{n \rightarrow \infty}{\longrightarrow} 0$. Existem portanto $\varepsilon>0$ e uma subsequência $\left(x_{n_{k}}\right)_{k \geq 1}$ de $\left(x_{n}\right)_{n \geq 1}$ com $\left\|x_{0}-x_{n_{k}}\right\| \geq \varepsilon$ para todo $k \geq 1$; pode-se concluir que $x_{n_{k}} \in \bar{\Omega}\left(\varepsilon, x_{0}\right), \forall k \geq 1$. Como $\left(2-\left\|x_{0}+x_{n_{k}}\right\|\right) \stackrel{k \rightarrow \infty}{\longrightarrow} 0$ garante que $\zeta\left(x_{0}, \varepsilon\right)=0$, segue que $\|\cdot\|$ não é localmente uniformemente convexa em $x_{0}$, impossível.

b) $\Rightarrow a)$ : Pela contra-reciproca, admitiremos que $\zeta\left(x_{0}, \varepsilon_{0}\right)=0$ para algum $0<\varepsilon_{0} \leq 2$. Existe portanto uma sequência $\left(z_{n}\right)_{n \geq 1} \subset$ $\mathcal{S}_{X}(\|\cdot\|)$ tal que $\left\|x_{0}-z_{n}\right\| \geq \varepsilon_{0}$ para todo $n \geq 1$ e é tal que $\left(2-\left\|x_{0}+z_{n}\right\|\right)_{n \geq 1} \stackrel{n \rightarrow \infty}{\longrightarrow} 0$. Logo $\left(z_{n}\right)_{n \geq 1}$ satisfaz as hipóteses presentes em b) mas é tal que $\left(\left\|x_{0}-z_{n}\right\|\right)_{n \geq 1}$ não converge para zero, o que é a negação de b) desejada.

- c) $\Rightarrow d)$ : imediato.

$d) \Rightarrow c): \operatorname{Para}\left(x_{n}\right)_{n \geq 1} \subset X \operatorname{com} 2\left(\left\|x_{0}\right\|^{2}+\left\|x_{n}\right\|^{2}\right)-\left\|x_{0}+x_{n}\right\|^{2} \stackrel{n \rightarrow \infty}{\longrightarrow} 0$ temos $\left\|x_{n}\right\| \stackrel{n \rightarrow \infty}{\longrightarrow}\left\|x_{0}\right\|$, uma vez que

$$
\begin{aligned}
0 & \leq\left(\left\|x_{0}\right\|-\left\|x_{n}\right\|\right)^{2}=2\left\|x_{0}\right\|^{2}+2\left\|x_{n}\right\|^{2}-\left(\left\|x_{0}\right\|+\left\|x_{n}\right\|\right)^{2} \leq \\
& \leq 2\left(\left\|x_{0}\right\|^{2}+\left\|x_{n}\right\|^{2}\right)-\left\|x_{0}+x_{n}\right\|^{2} \stackrel{n \rightarrow \infty}{\longrightarrow} 0 .
\end{aligned}
$$


Observando que assumimos $2\left(\left\|x_{0}\right\|^{2}+\left\|x_{n}\right\|^{2}\right)-\left\|x_{0}+x_{n}\right\|^{2} \stackrel{n \rightarrow \infty}{\longrightarrow} 0$, podemos agora concluir que $\left\|x_{0}+x_{n}\right\| \stackrel{n \rightarrow \infty}{\longrightarrow} 2$ e teremos, pela hipótese, que $\left\|x_{0}-x_{n}\right\| \stackrel{n \rightarrow \infty}{\longrightarrow} 0$ como desejado.

- $b) \Rightarrow d)$ : Suponha a sequência $\left(x_{n}\right)_{n \geq 1} \subset X$ tal que $\left\|x_{n}\right\| \stackrel{n \rightarrow \infty}{\longrightarrow} 1 \mathrm{e}$ $\left\|x_{0}+x_{n}\right\| \stackrel{n \rightarrow \infty}{\longrightarrow} 2$. Existe $N_{0} \geq 1$ tal que $\left\|x_{n}\right\|>0, \forall n \geq N_{0}$. Podemos assim definir a sequência $\left(y_{n}\right)_{n \geq 1} \subset \mathcal{S}_{X}(\|\cdot\|)$ por $y_{n}=0$ se $n<N_{0}$ e $y_{n}=x_{n} /\left\|x_{n}\right\|$ caso contrário. Mostremos que $\left(x_{0}+y_{n}\right)_{n \geq 1}$ e $\left(x_{0}+x_{n}\right)_{n \geq 1}$ têm mesmo limite (e segue que $\left\|x_{0}+y_{n}\right\| \stackrel{n \rightarrow \infty}{\longrightarrow} 2$ ).

De fato,

$$
\begin{aligned}
0 & \leq\left\|\left(x_{0}+y_{n}\right)-\left(x_{0}+x_{n}\right)\right\|=\left\|\frac{x_{n}}{\left\|x_{n}\right\|}-x_{n}\right\|= \\
& =\left\|\frac{x_{n}\left(1-\left\|x_{n}\right\|\right)}{\left\|x_{n}\right\|}\right\|=1-\left\|x_{n}\right\| \stackrel{n \rightarrow \infty}{\longrightarrow} 0 .
\end{aligned}
$$

Pela hipótese segue que $\left\|x_{0}-x_{n} /\right\| x_{n}\|\|=\left\|x_{0}-y_{n}\right\| \stackrel{n \rightarrow \infty}{\longrightarrow} 0 \mathrm{e}$ como $\left\|x_{n}\right\| \stackrel{n \rightarrow \infty}{\longrightarrow} 1$, podemos garantir que $\left\|x_{0}-x_{n}\right\| \stackrel{n \rightarrow \infty}{\longrightarrow} 0$, como desejado.

$d) \Rightarrow b):$ Imediato.

\subsection{Pontos extremais, expostos e a proprie- dade $A$}

O mesmo comentário presente no início da seção anterior aqui se aplica: antes de procedermos com os resultados de J.Lindenstrauss, desenvolveremos alguns conceitos e propriedades geométricas de espaços de Banach, que visam (parcialmente) caracterizar os espaços possuidores da propriedade $A$. A importância dos pontos extremais/[fortemente] expostos ficará evidente no decorrer da seção. 


\section{Definição 3.24 (pontos extremais)}

Seja $C$ um subconjunto convexo de um espaço de Banach $X$.

Dizemos que $x \in C$ é (ponto) extremal de $C$ se dados quaisquer $y, z \in C$ e $\alpha \in[0,1]$ tais que

$$
(1-\alpha) y+\alpha z=x
$$

tem-se necessariamente $x=y$ ou $x=z($ (e portanto $a=0$ ou $\alpha=1)$.

Geometricamente, constatamos que $x$ é extremal de $C$ quando qualquer segmento contido em $C$ que o contenha, o contém numa das extremidades.

A seguir daremos exemplos de espaços de Banach cujos conjuntos de pontos extremais são vazios ou constituem um subconjunto próprio da esfera unitária associada; logo a seguir, caracterizamos aqueles que têm como extremais todos os pontos de sua esfera.

Exemplo 3.25 A bola unitária de $\left(c_{0},\|\cdot\|\right.$ ) (norma usual) não tem pontos extremais.

De fato, considere o espaço de Banach $\left(c_{0},\|\cdot\|\right)$ (onde $\|\cdot\|$ é a norma usual de $c_{0}$ ) e a bola unitária $\mathcal{B}_{c_{0}}(\|\cdot\|)$.

Fixando qualquer $x=\left(x_{1}, x_{2}, \ldots\right) \in \mathcal{B}_{c_{0}}(\|\cdot\|)$, separemos em dois casos:

- Existe $N_{0} \geq 1$ para o qual $x_{n}=0, \forall n>N_{0}$.

Tome

$$
\begin{gathered}
a=\left(x_{1}, x_{2}, \ldots, x_{N_{0}}, \frac{1}{N_{0}+1}, \frac{1}{N_{0}+2}, \ldots\right) \mathrm{e} \\
b=\left(x_{1}, x_{2}, \ldots, x_{N_{0}},-\frac{1}{N_{0}+1},-\frac{1}{N_{0}+2}, \ldots\right) .
\end{gathered}
$$

Claro que $a$ e $b$ são elementos distintos de $\mathcal{B}_{c_{0}}(\|\cdot\|)$ e $x=(a+b) / 2$, distinto de ambos, não é ponto extremal da bola.

- Não existe $N_{0} \geq 1$ nas condições do caso anterior. Uma vez que existe $N_{1} \geq 1$ tal que $\left|x_{n}\right| \leq 1 / 2$ para todo $n>N_{1}$, tomamos

$$
\begin{gathered}
a=\left(x_{1}, x_{2}, \ldots, x_{n}, 0,2 \cdot x_{n+2}, 0,2 \cdot x_{n+4}, 0,2 \cdot x_{n+6}, \ldots\right) \quad \mathrm{e} \\
b=\left(x_{1}, x_{2}, \ldots, x_{n}, 2 \cdot x_{n+1}, 0,2 \cdot x_{n+3}, 0,2 \cdot x_{n+5}, \ldots\right) .
\end{gathered}
$$

Novamente $a$ e $b$ são elementos distintos de $\mathcal{B}_{c_{0}}(\|\cdot\|)$ e $x=(a+b)_{/}^{\prime} 2$, sendo distinto de ambos, não é extremal da bola. 
Exemplo 3.26 A bola unitária de $\left(l^{1},\|\cdot\|\right)$ (norma usual) admite como pontos extremais os elementos do conjunto $W=\left\{ \pm e_{i}\right\}_{i \geq 1}$ onde $\left\{\epsilon_{i}\right\}_{i \geq 1} \quad$ é a base de Schauder canônica do $l^{1}$.

Note de imediato que os pontos de $W$ são extremais da bola. Passamos a mostrar que qualquer ponto distinto destes não é. De fato, se o ponto em questão se encontra no interior da bola nada precisamos fazer; do contrário resta-nos analisar os pontos da esfera que têm (pelo menos) duas coordenadas não nulas. Fixado um ponto $x=\left(x_{i}\right)_{i \geq 1}$ nestas condições, sejam $1 \leq p<q$ os dois menores índices para os quais $x_{p}$ e $x_{q}$ são não nulos. Definindo $J=\{j \in \mathbb{N}: j \geq q\}$, fazendo

$$
t_{p}=\left|x_{p}\right| \quad \text { e } \quad t_{q}=\sum_{j \in J}\left|x_{j}\right|
$$

segue que $t_{p}, t_{q} \in(0,1)$ e são tais que $t_{p}+t_{q}=1$. Isto feito, observe que definindo $z=\left(0,0, \ldots, x_{p} /\left|x_{p}\right|, 0,0, \ldots\right)$ e $w=\left(0,0, \ldots, x_{q} / t_{q}, x_{q+1} / t_{q} \ldots\right)$, segue que $z, w$ são dois pontos distintos da esfera tais que

$$
x=t_{p} z+t_{q} w .
$$

Segue a não extremalidade do ponto $x$.

Exemplo 3.27 A bola unitária de $\left(L^{1}([0,1]),\|\cdot\|\right)$ (norma usual) nâo admite pontos extremais.

Considerando $[a, b] \subset[0,1]$ e $\chi_{[a, b]}$ a função característica dada por

$$
\chi[a, b](x)= \begin{cases}1 & \text { se } x \in[a, b] \\ 0 & \text { se } x \in[0,1] \backslash[a, b]\end{cases}
$$

observamos inicialmente que $f=0$ não é ponto extremal da bola. De fato, tomando

$$
f_{1}=\chi[0,1] \text { e } f_{2}=-\chi[0,1]
$$

temos ambos elementos distintos da bola e $f=\left(f_{1}+f_{2}\right) / 2$, distinto de ambos, garante sua não extremalidade. 
Consideremos agora qualquer $f \in \mathcal{B}_{L^{1}([0,1])}(\|\cdot\|) \backslash\{\boldsymbol{0}\}$. Como (por continuidade da funçào dada pela integral abaixo) existe $0<\alpha<1$ tal que

$$
\int_{0}^{\alpha}|f| d \mu=\frac{1}{2}\|f\|
$$

fazendo

$$
f_{1}=2 f \cdot \chi_{[0, \alpha]} \quad \text { e } \quad f_{2}=2 f \cdot \chi_{] \alpha, 1]}
$$

observe que $f_{1}$ e $f_{2}$ são elementos distintos da bola unitária e tais que $f=\left(f_{1}+f_{2}\right) / 2$. Como $f$ é distinto de ambos, segue portanto que $f$ não é ponto extremal da bola.

O importante teorema que se segue nos indica alguns casos nos quais podemos "reconstituir" um conjunto conhecendo apenas quem são seus pontos extremais. Como veremos nesta seção, Lindenstrauss prova um resultado análogo valendo-se do conceito de pontos expostos.

\section{Teorema 3.28 (Krein-Milman)}

Seja $(X,\|\cdot\|)$ um espaço de Banach. Todo subconjunto nâo vazio. convexo e compacto em $X$ coincide com a envoltória convexa fechada do conjunto de seus pontos extremais.

Prova: Vide por exemplo [Diestel2], 148.

Um interessante resultado nos mostra a estreita relação entre pontos extremais e a estrita convexidade de espaços de Banach :

\section{Teorema 3.29}

Um espaço de Banach $(X,\|\cdot\|)$ é estritamente convero se e somente se todo ponto da esfera unitária $\mathcal{S}_{X}(\|\cdot\|)$ é ponto extremal da bola unitária $\mathcal{B}_{X}(\|\cdot\|)$.

Prova: Suponhamos $(X,\|\cdot\|)$ estritamente convexo e $z_{0} \in \mathcal{S}_{X}(\|\cdot\|)$. Sejam $x_{0}, y_{0} \in \mathcal{B}_{X}(\|\cdot\|)$ tais que

$$
z_{0}=(1-t) x_{0}+t y_{0} \text { para algum } t \in[0,1] \text {. }
$$

Vamos mostrar que $z_{0}=x_{0}=y_{0}$. Como

$$
1=\left\|z_{0}\right\|=\left\|(1-t) x_{0}+t y_{0}\right\| \leq(1-t)\left\|x_{0}\right\|+t\left\|y_{0}\right\| \leq 1,
$$


segue que $\left\|(1-t) x_{0}+t y_{0}\right\|=(1-t)\left\|x_{0}\right\|+t\left\|y_{0}\right\|=1$ e de imediato temos $\left\|x_{0}\right\|=\left\|y_{0}\right\|=1$ (do contrário a última igualdade seria desigualdade estrita).

Pela estrita convexidade de $X^{\prime}$ sabemos que, provado $\left\|x_{0}+y_{0}\right\|=2$, teremos pela proposição $(3.10 \mathrm{~d})$ ) que $x_{0}=y_{0} \mathrm{e}$, consequentemente, $z_{0}=$ $x_{0}=y_{0}$.

Basta então mostrar que $\left\|x_{0}+y_{0}\right\|<2$ não se verifica. Se assim fosse,

$$
\begin{aligned}
1 & =\left\|z_{0}\right\|=\left\|(1-t) z_{0}+t z_{0}\right\|= \\
& =\left\|(1-t)\left[(1-t) x_{0}+t y_{0}\right]+t\left[(1-t) x_{0}+t y_{0}\right]\right\|= \\
& =\left\|(1-t)^{2} x_{0}+t(1-t)\left(y_{0}+x_{0}\right)+t^{2} y_{0}\right\| \leq \\
& \leq(1-t)^{2}+t(1-t)\left\|x_{0}+y_{0}\right\|+t^{2}< \\
& <t^{2}+2 t(1-t)+(1-t)^{2}=1,
\end{aligned}
$$

absurdo.

Reciprocamente, suponha que cada ponto de $\mathcal{S}_{X}(\|\cdot\|)$ seja extremal da bola $\mathcal{B}_{X}(\|\cdot\|)$ e tome quaisquer $x, y \in \mathcal{S}_{X}(\|\cdot\|)$ tais que $\|x+y\|=2$. Uma vez que o ponto $\xi=(x+y) / 2$ pertence à esfera ele é (por hipótese) extremal de $\mathcal{B}_{X}(\|\cdot\|)$ e, portanto, $\xi=x$ ou $\xi=y$. De qualquer forma tem-se (de $2 \xi=x+y)$ que $x=y$ e a proposição $(3.10 \mathrm{~d})$ ) garante a estrita convexidade de $(X,\|\cdot\|)$.

\section{Corolário 3.30}

Os espaços de Banach $\left(c_{0},\|\cdot\|\right),\left(l^{1},\|\cdot\|\right) \in\left(L^{1}([0,1]),\|\cdot\|\right)$ (normas usuais) não sâo estritamente convexos.

Prova: Consequência direta dos exemplos (3.25), (3.26) e (3.2 $⿱$ ) ), aliados ao teorema.

\section{Definição 3.31 (pontos expostos e fortemente expostos)}

Considere $C$ um subconjunto convexo de um espaço de Banach $(X,\|\cdot\|)$.

- Um ponto $\xi \in C$ é dito (um ponto) exposto de $C$ se

$$
\exists f \in X^{\prime} \quad \text { tal que } f(\xi)>f(x), \quad \forall x \in C, x \neq \xi .
$$

Note portanto que $\xi \in C$ é exposto se e só se existe um hiperplanosuporte $\mathcal{H}$ que contém $\xi$ e não contém qualquer outro ponto de $C$. 
- Um ponto $\xi \in C$ é dito (um ponto) fortemente exposto de C se

a) $\exists f \in X^{\prime}$ tal que $f(\xi)>f(x), \quad \forall x \in C, x \neq \xi$,

b) Dada $\left(x_{n}\right)_{n \geq 1} \subset C \operatorname{com} f\left(x_{n}\right) \stackrel{n \rightarrow \infty}{\longrightarrow} f(\xi)$, tem-se $\left\|x_{n}-\xi\right\| \stackrel{n \rightarrow \infty}{\longrightarrow} 0$.

\section{Proposição 3.32}

Seja C um subconjunto convero de um espaço de Banach $(X,\|\cdot\|)$. Todo ponto exposto de $C$ é extremal (de $C$ ).

Prova: Considere qualquer ponto $\xi$ exposto de $C$ e $f \in X^{\prime \prime}$ um funcional a ele associado e tal que

$$
f(\xi)>f(w), \quad \text { para todo } w \in C \backslash\{\xi\} \text {. }
$$

Sejam dois pontos $x, y \in C$ distintos e $0 \leq \alpha \leq 1$ tais que satisfaçam $\alpha x+(1-\alpha) y=\xi$. Então

$$
f(\xi)=\alpha f(x)+(1-\alpha) f(y)>f(w), \quad \forall w \in C \backslash\{\xi\} .
$$

Supondo por absurdo que $\xi \neq x$ e $\xi \neq y(0<\alpha<1)$, escolhendo $w=x$ concluimos que

$$
\alpha f(x)+(1-\alpha) f(y)>f(x) \quad \Rightarrow \quad f(y)>f(x) .
$$

Escolhendo agora $w=y$ segue que

$$
\alpha f(x)+(1-\alpha) f(y)>f(y)
$$

e teríamos $f(x)>f(y)$, absurdo.

Observação 3.33 Usaremos "colchetes" em alguns enunciados seguintes para evitar dividí-los em dois, ainda que a prova de uma das partes nâo seja imediata a partir da outra.

\section{Proposição 3.34}

Suponha $(X,\|\cdot\|)$ espaço de Banach estritamente flocalmente uniformemente] convero. Todo ponto da esfera $\mathcal{S}_{X}(\|\cdot\|)$ é ponto [fortemente] exposto da bola $\mathcal{B}_{X}(\|\cdot\|)$. 
Prova: Considere $\xi \in \mathcal{S}_{X}(\|\cdot\|)$ fixado. Uma vez que ele é nào nulo, segue do corolário (1.17) a existência de $f \in \mathcal{S}_{X^{\prime}}(\|\cdot\|) \operatorname{com} f(\xi)=\|\xi\|=\|f\|=1$.

Usando a estrita convexidade de $\mathrm{X}^{\prime}$ podemos garantir que o valor máximo de $f$ na esfera é assumido apenas em $\xi$ e, portanto,

$$
f(x)<f(\xi)=1, \forall x \in \mathcal{S}_{X}(\|\cdot\|) \backslash\{\xi\} .
$$

Considere agora qualquer ponto $z \in \mathcal{B}_{X}(\|\cdot\|) \backslash \mathcal{S}_{X}(\|\cdot\|)$. Pelo teorema (1.19) sabemos que

$$
\|z\|=\sup _{\varphi \in \mathcal{S}_{X^{\prime}}(\|\cdot\|)}|\varphi(z)| \geq|f(z)|
$$

e portanto $|f(z)|<1$.

Concluimos que $f(x)<f(\xi)=1, \forall x \in \mathcal{B}_{X}(\|\cdot\|) \backslash\{\xi\}$, garantindo que $\xi$ é ponto exposto da bola unitária.

Considerando agora como hipótese a local uniformidade convexa de $X$, passemos a mostrar que $\xi$ é fortemente exposto da bola.

Tomando então $\left(x_{n}\right)_{n \geq 1} \subset \mathcal{B}_{X}(\|\cdot\|)$ tal que $f\left(x_{n}\right) \stackrel{n \rightarrow \infty}{\longrightarrow} f(\xi)=1$, devemos mostrar que $\left\|x_{n}-\xi\right\| \stackrel{n \rightarrow \infty}{\longrightarrow} 0$. Uma vez que $f$ é linear e $f(\xi)=$ $\|\xi\|=1$ teremos

$2 \geq\|\xi\|+\left\|x_{n}\right\| \geq\left\|\xi+x_{n}\right\|=\|f\|\left\|\xi+x_{n}\right\| \geq f\left(\xi+x_{n}\right)=1+f\left(x_{n}\right) \stackrel{n \rightarrow \infty}{\longrightarrow} 2$

e podemos concluir que $\left\|\xi+x_{n}\right\| \stackrel{n \rightarrow \infty}{\longrightarrow} 2$.

Pela proposição (3.23 d)), mostrando que $\left\|x_{n}\right\| \stackrel{n \rightarrow \infty}{\longrightarrow} 1$ segue o almejado. Como para todo $n \geq 1$ temos

$$
1 \geq\left\|x_{n}\right\|=\sup _{\varphi \in \mathcal{S}_{X^{\prime}}(\|\cdot\|)}\left|\varphi\left(x_{n}\right)\right| \geq\left|f\left(x_{n}\right)\right| \stackrel{n \rightarrow \infty}{\longrightarrow} 1,
$$

segue que $\left\|x_{n}\right\| \stackrel{n \rightarrow \infty}{\longrightarrow} 1$ e encerramos o resultado.

\section{Exemplo 3.35 (nem todo exposto o é fortemente)}

Mostremos a existência de um espaço de Banach $(X,\|\cdot\|)$ que admite um ponto da esfera $\mathcal{S}_{X}(\|\cdot\|)$ exposto mas não fortemente exposto de um convexo $W$ que a contém. 
Considere o espaço de Banach $l^{2}$ munido de sua norma usual. Pelo teorema de Riesz (1.35) garante-se que todo elemento de $\left(l^{2}\right)^{\prime}$ pode ser dado por um $f=\left(a_{1}, a_{2}, \ldots\right) \in l^{2}$ tal que

$f(x)=\left(a_{1}, a_{2}, \ldots\right)\left(x_{1}, x_{2}, \ldots\right)=\sum_{k \geq 1} a_{i} \cdot x_{i} \quad$ para todo $x=\left(x_{1}, x_{2}, \ldots\right) \in l^{2}$.

Seja o conjunto $W$ definido como sendo

$$
W=\operatorname{conv}\left(\mathcal{B}_{l^{2}}(\|\cdot\|) \cup \bigcup_{k \geq 2}\left\{ \pm p_{k}\right\}\right)
$$

onde para todo $k \geq 2$ temos

$$
p_{k}=\left(1-\frac{1}{k}, 0,0, \ldots, 0,1,0, \ldots, 0\right),
$$

com o valor 1 na $k \underline{\text { a }}$ posição.

É imediato constatar que $W$ constitui um subconjunto convexo de $l^{2}$ que contém a bola unitária. Além disso, o ponto $\xi=(1,0,0, \ldots)$, pertencente à esfera unitária, é tal que $f(\xi)=1$ para o particular funcional $f=(1,0,0, \ldots) \in\left(l^{2}\right)^{\prime}$.

Como queremos

$$
f(\xi)>f(z) \quad, \quad \forall z \in W \backslash\{\xi\}
$$

para garantir que o funcional $f$ associa-se à exposição de $\xi$ em $W$, provemos que não existe ponto de $W \backslash\{\xi\}$ que tenha como primeira coordenada o valor 1 :

Suponhamos por absurdo que houvesse $\eta=\left(1, a_{2}, a_{3}, \ldots\right) \in W$ com $a_{j} \neq 0$ para algum $j \geq 2$. Pelo teorema (1.3) e da definição de $W$ podemos afirmar que existe subconjunto finito $I \subset \mathbb{N}$ em relação ao qual

$$
\eta=\sum_{i \in I} \alpha_{i} x_{i}
$$

onde $\left\{\alpha_{i}\right\}_{i \in I} \subset[0,1]$ é tal que $\sum_{i \in I} \alpha_{i}=1 \mathrm{e}$

$$
x_{i} \in \mathcal{B}_{l^{2}}(\|\cdot\|) \cup \bigcup_{k \geq 2}\left\{ \pm p_{k}\right\} \quad, \forall i \geq 1 .
$$


Uma vez que

$$
\|\eta\|^{2}=\left\|\left(1, a_{2}, \ldots, a_{j}, \ldots\right)\right\|^{2}=1+\sum_{i \geq 2}\left|a_{i}\right|^{2} \geq 1+\left|a_{j}\right|^{2}>1,
$$

então ${ }^{6}$ existe $m \in I$ tal que $x_{m} \notin \mathcal{B}_{l^{2}}(\|\cdot\|)$ e, assim sendo, $x_{m}= \pm p_{N_{m}}$ para algum $N_{m} \geq 2$.

Consideremos o subconjunto de índices (não vazio) $J \subset I$ constituido pelos elementos de $\left\{x_{i}\right\}_{i \in I}$ que não se encontram na bola unitária de $l^{2}$. Podemos escrever

$$
\left(1, a_{2}, a_{3}, \ldots\right)=\eta=\sum_{j \in J} \pm \alpha_{j} p_{N_{j}}+\sum_{i \in I \backslash J} \alpha_{i} x_{i} .
$$

Observando agora que, se tomarmos da igualdade obtida apenas a primeira coordenada, teremos

$$
1=\sum_{j \in J} \pm \alpha_{j}\left(1-\frac{1}{N_{j}}\right)+\sum_{i \in I \backslash J} \alpha_{i} x_{i, 1},
$$

segue que, tomando o módulo,

$$
\begin{aligned}
1 & =\left|\sum_{j \in J} \pm \alpha_{j}\left(1-\frac{1}{N_{j}}\right)+\sum_{i \in I \backslash J} \alpha_{i} x_{i, 1}\right| \leq \sum_{j \in J} \alpha_{j}\left(1-\frac{1}{N_{j}}\right)+\sum_{i \in I \backslash J} \alpha_{i}\left|x_{i, 1}\right|< \\
& <\sum_{j \in J} \alpha_{j}+\sum_{i \in I \backslash J} \alpha_{i}=1,
\end{aligned}
$$

absurdo.

Como para qualquer $z=\left(\tilde{z}_{1}, z_{2}, \ldots\right) \in W$ podemos escrever

$$
f(z)=(1,0,0, \ldots)\left(z_{1}, z_{2}, z_{3}, \ldots\right)=z_{1},
$$

a afirmação recém-provada é suficiente para garantir que $\xi$ é um ponto exposto de $W$.

${ }^{6}$ De fato, caso tivéssemos $x_{i} \in \mathcal{B}_{l^{2}}(\|\cdot\|)$ para todo $i \in I$ então

$$
\|\eta\|=\left\|\sum_{i \in I} \alpha_{i} x_{i}\right\| \leq \sum_{i \in I} \alpha_{i}\left\|x_{i}\right\| \leq \sum_{i \in I} \alpha_{i}=1,
$$

o que não é verdade. 
Intencionando analisar a exposição forte, mostraremos inicialmente que todo funcional de $\left(l^{2}\right)^{\prime}$ que expòe $\xi \mathrm{em} W$ coincide com $f$.

De fato, considerando $g=\left(b_{1}, b_{2}, \ldots\right) \in l^{2} \backslash\{0\}$ nestas condiçòes podemos, sem perda de generalidade, assumir que $\|g\|=1$ (norma usual). Uma vez que $g(\xi)=b_{1}>g(w)$, para todo $w \in W \backslash\{\xi\}$, tomando para cada $k \geq 2$ o vetor $w_{k}=(0,0, \ldots, 0,1,0, \ldots)$ onde o valor 1 ocupa a $k$ â posição, temos de imediato $b_{1}>g\left(w_{j}\right)=b_{j}$ para todo $j \geq 2$. De $g \neq 0$ podemos garantir que existe $m \geq 1$ tal que $\left|b_{m}\right|>0$. Como $b_{1}>g\left( \pm w_{k}\right)= \pm b_{k}$ para todo $k \geq 2$, segue que $b_{1}>0$.

Uma vez que $\|g\|=1$, vamos ter $b_{1}=1$ ou $0<b_{1}<1$. Se $b_{1}=1$ temos $b_{j}=0$ para todo $j \geq 2$ e consequentemente $g=f$ como desejado. Caso $0<b_{1}<1$, então

$$
1=\|g\|=|g(\xi)|=b_{1}<1,
$$

absurdo.

Resta portanto apenas garantir que a exposição de $\xi$ pela $f$ não é forte.

De fato, tomando $\left(x_{k}\right)_{k \geq 1} \subset W$ dado por $x_{k}=p_{k}$ para todo $k \geq 1$, teremos para $f=(1,0,0, \ldots) \in\left(l^{2}\right)^{\prime}$ :

$$
f\left(x_{k}\right)=(1,0,0, \ldots)\left(1-\frac{1}{k}, 0,0, \ldots, 0,1,0, \ldots,\right)=1-\frac{1}{k} \stackrel{k \rightarrow \infty}{\longrightarrow} 1=f(\xi)
$$

porém uma vez que

$$
\left\|x_{k}-\xi\right\|^{2}=\left\|\left(1-\frac{1}{k}, 0, \ldots, 0,1,0,0, \ldots\right)-(1,0,0 \ldots)\right\|^{2} \geq 1+\frac{1}{k^{2}} \stackrel{k \rightarrow \infty}{\nrightarrow} 0,
$$

certamente $\xi$ não é ponto fortemente exposto de $W$.

\section{Proposição $\mathbf{3 . 3 6}$}

Considere $(X,\|\cdot\|)$ espaço de Banach. O conjunto dos pontos [fortemente] expostos da bola unitária $\mathcal{B}_{X}(\|\cdot\|)$ é simétrico (em relação à origem).

Prova: Basta mostrar que, se $\xi$ é ponto [fortemente] exposto de $\mathcal{B}_{X}(\|\cdot\|)$ então $-\xi$ também será. 
Consideremos inicialmente $\xi \in \mathcal{B}_{X}(\|\cdot\|)$ ponto exposto da bola unitária $\mathcal{B}_{X}(\|\cdot\|)$. Seja $f \in X^{\prime \prime}$ tal que $f(\xi)>f(y), \forall y \in \mathcal{B}_{X}(\|\cdot\|) \backslash\{\xi\}$.

Sendo $\mathcal{B}_{X}(\|\cdot\|)$ um conjunto simétrico temos, a partir da expressào acima, que

$$
f(\xi)>f(-x), \forall x \in \mathcal{B}_{X}(\|\cdot\|) \backslash\{-\xi\} .
$$

Tomando então $g \in X^{\prime \prime}$ dado por $g=-f$, tem-se

$g(-\xi)=-f(-\xi)=f(\xi)>f(-x)=-g(-x)=g(x), \forall x \in \mathcal{B}_{X}(\|\cdot\|) \backslash\{-\xi\}$

e portanto $-\xi$ é ponto exposto da bola unitária $\mathcal{B}_{X}(\|\cdot\|)$.

No caso de $\xi \in \mathcal{B}_{X}(\|\cdot\|)$ ser (como hipótese adicional) fortemente exposto, considere o funcional $f \in X^{\prime}$ dado e uma sequência $\left(x_{n}\right)_{n \geq 1} \subset \mathcal{B}_{X}(\|\cdot\|)$ tal que $f\left(x_{n}\right) \stackrel{n \rightarrow \infty}{\longrightarrow} f(\xi)$ implica em $\left\|x_{n}-\xi\right\| \stackrel{n \rightarrow \infty}{\longrightarrow} 0$.

Para a mesma $g=-f$ anterior, $g\left(x_{n}\right) \stackrel{n \rightarrow \infty}{\longrightarrow} g(\xi)$ garante $-f\left(x_{n}\right) \stackrel{n \rightarrow \infty}{\longrightarrow}$ $-f(\xi)$ e, portanto, $f\left(x_{n}\right) \stackrel{n \rightarrow \infty}{\longrightarrow} f(\xi)$. Segue que $\left\|x_{n}-\xi\right\| \stackrel{n \rightarrow \infty}{\longrightarrow} 0$ e concluimos ser $-\xi$ fortemente exposto de $\mathcal{B}_{X}(\|\cdot\|)$.

\section{Corolário 3.37}

Sejam $(X,\|\cdot\|)$ um espaço de Banach estritamente [localmente uniformemente] convero e $\Omega$ o conjunto dos pontos [fortemente] expostos da bola unitária. Então a envoltória convexa fechada $C$ de $\Omega$ constitui um subconjunto simétrico de $\mathcal{B}_{X}(\|\cdot\|)$ que contém a origem.

Prova: Pela proposição (3.34) sabemos que $\mathcal{S}_{X}(\|\cdot\|) \subset \Omega$.

Uma vez que $\mathcal{S}_{X}(\|\cdot\|)$ é simétrica em relação à origem e podemos escrever

$$
0=\frac{1}{2} x+\frac{1}{2}(-x) \text { para qualquer } x \in \mathcal{S}_{X}(\|\cdot\|),
$$

segue que todo convexo que contém $\Omega$ contém também a origem e garante-se então $0 \in C$.

Como por definição tem-se $\Omega \subset \mathcal{B}_{X}(\|\cdot\|)$ e observando que a própria bola unitária constitui subconjunto convexo e fechado em $X$ que contém seus pontos [fortemente] expostos, segue que $C \subset \mathcal{B}_{X}(\|\cdot\|)$ e, pela proposição (3.36), a simetria de $\Omega$ nos permite garantir a simetria da envoltória. 


\section{Proposição 3.38}

Seja $(X,\|\cdot\|)$ um espaço de Banach estritamente [localmente uniformemente] convexo. Se a envoltória convexa fechada $C$ dos pontos [fortemente] expostos de $\mathcal{B}_{X}(\|\cdot\|)$ não coincidir com a própria bola unitária, existem $f \in \mathcal{S}_{X^{\prime}}(\|\cdot\|)$ e $\delta>0$ tais que

$$
|f(x)|<1-\delta, \forall x \in C .
$$

Prova: Dado $\xi \in \mathcal{B}_{X}(\|\cdot\|) \backslash C$, considere $C$ e $\{\xi\}$ como sendo, respectivamente, os conjuntos fechado e compacto não vazios, disjuntos e convexos associados à forma geométrica de Hahn-Banach (teorema (1.16)). Existem portanto um funcional não nulo $g \in X^{\prime}$ e um real $\alpha$ tais que $g(x)<\alpha<g(\xi)$, para todo $x \in C$.

Como $\|g\|>0$ teremos

$$
\sup _{x \in C} \frac{g(x)}{\|g\|} \leq \frac{\alpha}{\|g\|}<\frac{g(\xi)}{\|g\|} \leq \sup _{x \in \mathcal{B}_{X}(\|\cdot \cdot\|)} \frac{g(x)}{\|g\|}=1,
$$

donde segue que $f=g /\|g\| \in \mathcal{S}_{X^{\prime}}(\|\cdot\|)$ e satisfaz

$$
\sup _{x \in C} f(x) \leq \alpha /\|g\|<1 .
$$

Garantimos que existe $\delta>0$ tal que $\sup _{x \in C} f(x) \leq 1-\delta$.

Sendo $C$ simétrico (corolário (3.37)), podemos garantir para todo $x \in C$ que

$$
-f(x)=f(-x) \leq \sup _{z \in C} f(z)<1-\delta
$$

e portanto $\sup _{x \in C}|f(x)|<1-\delta$ como desejado.

\section{Proposição 3.39}

Sejam $(X,\|\cdot\|)$ um espaço de Banach, $(Y,\|\cdot\|)$ um espaço normado $\epsilon$

$$
T:(X,\|\cdot\|) \longrightarrow(Y,\|\cdot\|)
$$

um isomorfismo (nâo nulo) sobre sua imagem. Se a norma de $Y$ é estritamente [localmente uniformemente] convexa quando restrita ao subespaço $T(X)$ e $T$ atinge sua norma num ponto $\xi \in \mathcal{S}_{X}(\|\cdot\|)$, então $\xi$ é ponto [fortemente] exposto de $\mathcal{B}_{X}(\|\cdot\|)$. 
Prova: Pelo teorema (1.1T) podemos tomar $g \in \mathcal{S}_{Y}(\|\cdot\|)$ tal que $g(T \xi)=\|T \xi\|=\|T\|>0$.

Passamos a mostrar que $f=g \circ T \in X^{\prime \prime}$ é o funcional que cumpre as propriedades associadas à [forte] exposição de $\xi$ em $\mathcal{B}_{X}(\|\cdot\|)$.

- Vamos inicialmente fazer o caso de $\left(T\left(X^{\prime}\right),\|\cdot\|\right)$ estritamente convexo. Mostraremos que $\xi$ é ponto exposto da bola unitária $\mathcal{B}_{X}(\|\cdot\|)$ associado ao funcional $f$, provando que

$$
f(\xi)>f(x), \forall x \in \mathcal{B}_{X}(\|\cdot\|) \backslash\{\xi\} .
$$

Suponha inicialmente por absurdo que houvesse $\eta \in \mathcal{B}_{X}(\|\cdot\|)$ tal que $f(\eta)>f(\xi)=\|T\|$. Se assim fosse, o teorema (1.19) garantiria

$$
\|T(\eta)\|=\sup _{\varphi \in \mathcal{B}_{Y^{\prime}}(\|\cdot\|)}|\varphi(T \eta)| \geq|g(T \eta)|=f(\eta)>f(\xi)=\|T\|,
$$

o que certamente não é possível.

Suponha agora existir $\xi_{1} \in \mathcal{B}_{X}(\|\cdot\|)$ tal que $f\left(\xi_{1}\right)=f(\xi)$. Neste caso

$$
g\left(T \xi_{1}\right)=f\left(\xi_{1}\right)=f(\xi)=g(T \xi)=\|T\| .
$$

Uma vez que $\left\|T \xi_{1}\right\| \leq\|T\|$, sabemos que $T \xi_{1} /\|T\| \in \mathcal{B}_{Y}(\|\cdot\|)$ e podemos dividir o problema em dois:

- Caso $T \xi_{1} /\|T\| \in \mathcal{S}_{Y}(\|\cdot\|)$ : Pela expressão (3.15) vem

$$
g\left(\frac{T \xi_{1}}{\|T\|}\right)=g\left(\frac{T \xi}{\|T\|}\right)=1=\|g\|,
$$

donde segue ${ }^{7}$ que o funcional $g \in Y^{\prime}$, assumindo seu valor máximo num único ponto da esfera, nos garante que $T\left(\xi_{1}\right)=T(\xi)$. Como $T$ é um isomorfismo, pela sua injetividade podemos concluir que $\xi_{1}=\xi$, como desejado.

\footnotetext{
${ }^{7}$ Proposição $\left.(3.10, \mathrm{~b})\right)$, associada à estrita convexidade.
} 
- Caso $T \xi_{1} /\|T\| \in \mathcal{B}_{Y}(\|\cdot\|) \backslash \mathcal{S}_{Y}(\|\cdot\|)$ : Valendo-nos do teorema (1.19) e da expressão (3.15) temos

$$
\left\|\frac{T \xi_{1}}{\|T\|}\right\|=\sup _{\varphi \in \mathcal{B}_{Y^{\prime}}(\|\cdot\|)}\left|\varphi\left(\frac{T \xi_{1}}{\|T\|}\right)\right| \geq\left|g\left(\frac{T \xi_{1}}{\|T\|}\right)\right|=\left|g\left(\frac{T \xi}{\|T\|}\right)\right|=1,
$$

contradição que garante não haver ponto $T \xi_{1} /\|T\|$ pertencente ao interior da bola unitária.

- Considerando-se agora a hipótese de $(T(X),\|\cdot\|)$ ser localmente uniformemente convexo, passamos a garantir que $f$ garante a forte exposição de $\xi$ em $\mathcal{B}_{X}(\|\cdot\|)$ :

Tomando uma sequência qualquer $\left(x_{n}\right)_{n \geq 1} \subset \mathcal{B}_{X}(\|\cdot\|)$ tal que

$$
g\left(T x_{n}\right)=f\left(x_{n}\right) \stackrel{n \rightarrow \infty}{\longrightarrow} f(\xi)=g(T \xi)=\|T\|,
$$

segue que $g\left(T x_{n}\right)-\|T\| \stackrel{n \rightarrow \infty}{\longrightarrow} 0$ ou, equivalentemente, que

$$
g\left(T x_{n}\right)+\|T\| \stackrel{n \rightarrow \infty}{\longrightarrow} 2\|T\| .
$$

Isto feito e de

$$
\begin{aligned}
\|T\|+g\left(T x_{n}\right) & =g(T \xi)+g\left(T x_{n}\right)= \\
& =g\left(T \xi+T x_{n}\right) \leq\|g\|\left\|T \xi+T x_{n}\right\|= \\
& =\left\|T \xi+T x_{n}\right\| \leq\|T \xi\|+\left\|T x_{n}\right\| \leq 2\|T\|
\end{aligned}
$$

concluimos que $\left\|T \xi+T x_{n}\right\| \stackrel{n \rightarrow \infty}{\longrightarrow} 2\|T\|$ e $\left\|T x_{n}\right\|+\|T \xi\| \stackrel{n \rightarrow \infty}{\longrightarrow} 2\|T\|$ ou, equivalentemente, $\left\|T x_{n}\right\| \stackrel{n \rightarrow \infty}{\longrightarrow}\|T \xi\|$ (uma vez que $\|T \xi\|=\|T\|$ ).

Da local uniformidade convexa da norma $\|\cdot\|$ no ponto $T \xi$ segue portanto pela proposição $(3.23 \mathrm{~d}))$ que $\left\|T \xi-T x_{n}\right\| \stackrel{n \rightarrow \infty}{\longrightarrow} 0 \mathrm{e}$, usando o fato de que $T$ é isomorfismo sobre sua imagem, existe uma constante $c>0$ tal que

$$
0 \leq\left\|\xi-x_{n}\right\| \leq c\left\|T \xi-T x_{n}\right\| \stackrel{n \rightarrow \infty}{\longrightarrow} 0
$$

garantindo então $\left\|\xi-x_{n}\right\| \stackrel{n \rightarrow \infty}{\longrightarrow} 0$, como desejado. 


\section{Lema 3.40}

Dado um espaço de Banach $(X, \||\cdot|||)$ estritamente [localmente uniformemente] convexo, o espaço vetorial $Y=X \times \mathbb{R}$ munido da aplicaçào definida por

$$
\|(x, r)\|=\left(\||x|\|^{2}+r^{2}\right)^{1 / 2},
$$

constitui um espaço normado estritamente [localmente uniformemente] convexo.

Prova: Vejamos inicialmente que $\|\cdot\|: Y \rightarrow \mathbb{R}$ constitui uma norma em $Y$, mostrando para tanto a única propriedade nào imediata: a desigualdade triangular.

$$
\begin{aligned}
& \|(x+y, r+s)\|^{2}=\|x+y\| \|^{2}+(r+s)^{2} \leq\left(\||| x|\|+\|||y| \mid)^{2}+r^{2}+s^{2}+2 r s=\right. \\
& =\left\|\left.|| x\left|\left\|^{2}+r^{2}+\right\|\right||y|\right|^{2}+s^{2}+2(\||| x|||\cdot|\| y \|||+r s) \leq\right. \\
& \leq\|(x, r)\|^{2}+\|(y, s)\|^{2}+2\left[\left(\|\| x \|^{2}+r^{2}\right)\left(\|\| y\|\|^{2}+s^{2}\right)\right]^{1 / 2}= \\
& =\|(x, r)\|^{2}+\|(y, s)\|^{2}+2\left(\|\mid x\| \|^{2}+r^{2}\right)^{1 / 2}\left(\|\| y \|^{2}+s^{2}\right)^{1 / 2}= \\
& =\|(x, r)\|^{2}+\|(y, s)\|^{2}+2(\|(x, r)\| \cdot\|(y . s)\|)= \\
& =(\|(x, r)\|+\|(y, s)\|)^{2}
\end{aligned}
$$

como desejado.

Passemos a mostrar que $(Y,\|\cdot\|)$ é estritamente convexo quando $(X,|\|\cdot\||)$ o for.

Considere $\xi=(x, r)$ e $\eta=(y, s)$ elementos de $\mathcal{S}_{\mathrm{Y}}(\|\cdot\|)$ tais que $\|\xi+\eta\|=2$. Pela proposição $(3.10 \mathrm{~d}))$ basta provarmos que $\xi=\eta$.

Segue das hipóteses que

$$
\|\| x\left\|\left.\right|^{2}+r^{2}=1,\right\| y\|\|^{2}+s^{2}=1 \quad \text { e } \quad\|x+y\| \|^{2}+(r+s)^{2}=2^{2} .
$$

Trocando ||$|\cdot|||$ por $|\cdot|$ para não sobrecarregar a notação. temos

$$
|x+y|^{2}+(r+s)^{2}-2\left(|x|^{2}+r^{2}\right)-2\left(|y|^{2}+s^{2}\right)=0 .
$$

Pela desigualdade triangular temos que

$$
(|x|+|y|)^{2}+(r+s)^{2}-2\left(|x|^{2}+r^{2}+|y|^{2}+s^{2}\right) \geq 0
$$


e expandindo esta expressão obtemos facilmente

$$
\begin{gathered}
-\left[(|x|-|y|)^{2}+(r-s)^{2}\right]= \\
=(|x|+|y|)^{2}+(r+s)^{2}-2\left(|x|^{2}+r^{2}+|y|^{2}+s^{2}\right) \geq 0 .
\end{gathered}
$$

Como o lado esquerdo (inicial) é certamente não positivo segue da última desigualdade que ele é nulo e, portanto, $\left(|x|^{2}-|y|^{2}\right)^{2}+(r-s)^{2}=0$. Voltando à notaçào original temos então

$$
r=s=p \leq 1 \quad \text { e } \quad\|x\||=\|\mid\| y \|=a \geq 0 .
$$

Caso $a=0$ nada resta a fazer; do contrário $\||x|\|=\||| y \mid\|=a>0$ e da expressào (3.16) podemos escrever $a^{2}+p^{2}=1$ e $\|x+y\| \|^{2}+(2 p)^{2}=4$. Portanto

$$
\|x+y\| \|=2 \sqrt{1-p^{2}}=2 a
$$

e a observação (3.11) nos garante $x=y$ como desejado.

Mostraremos agora que $(Y,\|\cdot\|)$ é localmente uniformemente convexo quando $(X,|||\cdot|||)$ o for.

Considere qualquer $\xi=(x, r) \in Y$ fixado. Tomando a sequência $\left(\xi_{n}\right)_{n \geq 1}=$ $\left(\left(x_{n}, r_{n}\right)\right)_{n \geq 1} \subset X \times \mathbb{R}$ tal que

$$
2\left(\|\xi\|^{2}+\left\|\xi_{n}\right\|^{2}\right)-\left\|\xi+\xi_{n}\right\|^{2} \stackrel{n \rightarrow \infty}{\longrightarrow} 0,
$$

basta mostrar que $\left\|\xi-\xi_{n}\right\| \stackrel{n \rightarrow \infty}{\longrightarrow} 0$.

De fato, trocando ||$|\cdot|||$ por $|\cdot|$ para não sobrecarregar a notação, como

$$
\begin{gathered}
2\left(|x|^{2}+r^{2}+\left|x_{n}\right|^{2}+r_{n}^{2}\right)-\left(\left|x+x_{n}\right|^{2}+\left(r+r_{n}\right)^{2}\right)= \\
=2\left(|x|^{2}+\left|x_{n}\right|^{2}\right)-\left|x+x_{n}\right|^{2}+2\left(r^{2}+r_{n}^{2}\right)-\left(r+r_{n}\right)^{2}= \\
=2\left(|x|^{2}+\left|x_{n}\right|^{2}\right)-\left|x+x_{n}\right|^{2}+\left(r-r_{n}\right)^{2},
\end{gathered}
$$

segue da hipótese que

$$
2\left(|x|^{2}+\left|x_{n}\right|^{2}\right)-\left|x+x_{n}\right|^{2}+\left(r-r_{n}\right)^{2} \stackrel{n \rightarrow \infty}{\longrightarrow} 0 .
$$


Como trivialmente $2\left(|x|^{2}+\left|x_{n}\right|^{2}\right)-\left|x+x_{n}\right|^{2} \geq 0, \forall n \geq 1$ temos, pela expressào (3.17), que $\left(r-r_{n}\right)^{2} \stackrel{n \rightarrow \infty}{\longrightarrow} 0$ e, portanto, $r_{n} \stackrel{n \rightarrow \infty}{\longrightarrow} r$. Sabendo disto e voltando para a notaçào original segue. novamente pela mesma expressão, que

$$
2\left(\left.\left\||| x||^{2}+\right\|\left|x_{n}\right|\right|^{2}\right)-\left\|\left|x+x_{n}\right|\right\|^{2} \stackrel{n \rightarrow \infty}{\longrightarrow} 0 .
$$

Sendo ||$|\cdot|||$ localmente uniformemente convexa (em $x$ ) segue da proposição $(3.23 \mathrm{c}))$ que $\|\left|x-x_{n}\right| \mid \stackrel{n \rightarrow \infty}{\longrightarrow} 0$.

Finalmente observando que $r_{n} \stackrel{n \rightarrow \infty}{\longrightarrow} r$ e $\left\|x-x_{n}\right\| \stackrel{n \rightarrow \infty}{\longrightarrow} 0$ temos, pela continuidade das aplicações envolvidas,

$$
\left\|\xi-\xi_{n}\right\|=\left(\left\||| x-x_{n}\right\|^{2}+\left(r-r_{n}\right)^{2}\right)^{1 / 2} \stackrel{n \rightarrow \infty}{\longrightarrow} 0
$$

como desejado.

Vejamos agora como Lindenstrauss definiu as "propriedades $A$ e $B$ ", e iniciemos o estudo por ele desenvolvido em relaçào à elas $^{8}$.

\section{Definição 3.41 (as propriedades $A$ e $B$ de Lindenstrauss)}

- (Propriedade A) Considere $X$ um espaço de Banach. Dizemos que X' tem a propriedade $A$ se $\mathcal{N} \mathcal{A}(X, Y)$ é denso em $\mathcal{L}(X, Y)$, para qualquer espaço de Banach $Y$ dado.

- (Propriedade B) Considere $Y$ um espaço de Banach. Dizemos que $Y$ tem a propriedade $B$ se $\mathcal{N} \mathcal{A}(X, Y)$ é denso em $\mathcal{L}(X, Y)$, para qualquer espaço de Banach X' dado.

Observe que os resultados estudados até aqui nos permitem afirmar que todo espaço reflexivo tem a propriedade $A$ e que o espaço de Banach $(\mathbb{R},|\cdot|)$ tem a propriedade $B$.

\footnotetext{
${ }^{8}$ Faremos neste capítulo resultados associados somente com a propriedade $A$. A razão disto se deve ao fato de deixarmos para explorar a propriedade $B$ no capítulo seguinte, no qual o espaço de Gowers é de grande importancia.
} 


\section{Teorema 3.42}

Considere $(X,\|\cdot\|)$ um espaço de Banach com a propriedade A. Se $X$ é isomorfo a um espaço estritamente [localmente uniformemente] convexo, então $\mathcal{B}_{X}(\|\cdot\|)$ coincide com a envoltória convexa fechada de seus pontos [fortemente] expostos.

Prova: Considere $C$ a envoltória convexa fechada dos pontos [fortemente] expostos da bola unitária $\mathcal{B}_{X}(\|\cdot\|)$. Como $C \subset \mathcal{B}_{X}(\|\cdot\|)$ e supondo por absurdo que a inclusão seja própria, valendo-nos da proposiçào (3.38) garantimos as existências de $f \in \mathcal{S}_{X^{\prime}}(\|\cdot\|)$ e $\delta>0$ tais que $|f(x)|<1-\delta$ para todo $x \in C$.

O isomorfismo dado na hipótese nos permite considerar uma norma ||$|\cdot|||$ estritamente [localmente uniformemente] convexa em $X$ equivalente à $\|\cdot\|$ dada e em relação a qual tenhamos $\|x\|\|\leq\| x \|$ para todo $x$ em $\mathrm{X}$. Pelo lema anterior, o espaço vetorial $Y=X \times \mathbb{R}$ munido da aplicaçào definida por

$$
\|(x, r)\|=\left(\||| x \mid\|^{2}+r^{2}\right)^{1 / 2},
$$

constitui um espaço normado estritamente [localmente uniformemente] convexo.

Fixemos uma constante $M>\max \{2 / 3,2 / \delta\}$ e considere o operador $V: X \rightarrow Y$ dado por

$$
V(x)=(x, M \cdot f(x)), \forall x \in X .
$$

Tem-se facilmente que $V$ é linear e injetivo. Passemos a mostrar que constitui um isomorfismo sobre sua imagem. De \|\|$x\|\| \leq\|x\|$ e $\|f\|=1$ temos

$$
\begin{aligned}
\|V x\| & =\|(x, M \cdot f(x))\|=\left(\|\| x\|\|^{2}+M^{2} \cdot f^{2}(x)\right)^{1 / 2} \leq \\
& \leq\left(\|x\|\left\|^{2}+M^{2}\right\| f\left\|^{2}\right\| x \|^{2}\right)^{1 / 2}=\left(\|\| x\|\|^{2}+M^{2}\|x\|^{2}\right)^{1 / 2} \leq \\
& \leq\left(\|x\|^{2} \cdot\left(1+M^{2}\right)\right)^{1 / 2}=\|x\| \cdot\left(1+M^{2}\right)^{1 / 2}
\end{aligned}
$$

e observando a existência de $c>0$ tal que $\|x\| \leq c \cdot\|x\| \|$ (pela equivalência de $\|\cdot\|$ e $\||\cdot|\|)$, podemos escrever

$$
\|V x\| \leq c \cdot\left(1+M^{2}\right)^{1 / 2} \cdot\|x\| \|, \forall x \in X .
$$


Uma vez que $V$ é limitada (portanto contínua), resta provarmos que $V(X)$ é subespaço fechado de $Y$ (logo é Banach) para podermos utilizar o teorema (1.22).

De fato, considere a sequência $\left(V\left(x_{n}\right)\right)_{n \geq 1}=\left(\left(x_{n}, M \cdot f\left(x_{n}\right)\right)_{n \geq 1}\right.$ convergente para digamos $y_{0}=\left(x_{0}, t_{0}\right) \in X \times \mathbb{R}$. Temos

$\left.||\left|x_{n}-x_{0}\right|\right|^{2}+\left|M \cdot f\left(x_{n}\right)-t_{0}\right|^{2}=\left\|\left(x_{n}-x_{0}, M \cdot f\left(x_{n}\right)-t_{0}\right)\right\|^{2} \stackrel{n \rightarrow \infty}{\longrightarrow} 0$

garantindo que $\left\|\mid x_{n}-x_{0}\right\| \stackrel{n \rightarrow \infty}{\longrightarrow} 0$ e $M \cdot f\left(x_{n}\right) \stackrel{n \rightarrow \infty}{\longrightarrow} t_{0}$. Da continuidade da $f$ e da unicidade do limite segue que $M \cdot f\left(x_{0}\right)=t_{0}$ e, portanto, $y_{0}=V\left(x_{0}\right)$, provando o desejado.

Valendo-nos do teorema (1.22) segue que a inversa $V^{-1}: V(X) \rightarrow X$ também é (linear e) contínua, o que prova ser $V$ isomorfismo sobre sua imagem.

Vejamos agora duas propriedades adicionais associadas ao operador $V$ que nos mostram que ele não atinge sua norma em um ponto de $C$.

- $\|V\| \geq M$ :

Uma vez que

$$
\begin{aligned}
\|V x\| & =\|(x, M \cdot f(x))\|=\left(\||| x \mid\|^{2}+M^{2} \cdot f^{2}(x)\right)^{1 / 2} \geq \\
& \geq\left(M^{2} \cdot f^{2}(x)\right)^{1 / 2}=M \cdot|f(x)|,
\end{aligned}
$$

como $\|f\|=1$ segue o desejado.

- $\|V x\| \leq\left(1+(M-2)^{2}\right)^{1 / 2} \leq M-1, \forall x \in C \quad$ :

De fato, fixado qualquer $x \in C$ temos $|f(x)|<1-\delta$ e então

$$
\begin{aligned}
\|V x\| & =\left(\||x|\|^{2}+M^{2} \cdot f^{2}(x)\right)^{1 / 2} \leq\left(\||| x||^{2}+(M \cdot|f(x)|)^{2}\right)^{1 / 2} \leq \\
& \leq\left(\|x \mid\|^{2}+(M \cdot(1-\delta))^{2}\right)^{1 / 2}=\left(\left\|\left|\|x \mid\|^{2}+(M-M \cdot \delta)^{2}\right)^{1 / 2} .\right.\right.
\end{aligned}
$$

Como $M>2 / \delta$ garantimos $-M \cdot \delta<-2$ e, valendo-nos do fato de que $\||x|\| \leq\|x\| \leq 1$ (pois $C \subset \mathcal{B}_{X}(\|\cdot\|)$ ), teremos

$$
\|V x\| \leq\left(\|x\|^{2}+(M-2)^{2}\right)^{1 / 2} \leq\left(1+(M-2)^{2}\right)^{1 / 2} .
$$


Observando que $0<\delta<1$ e $M>2 / \delta$ garantem $M>2$ ou, equivalentemente, $-2 M+4<0$, teremos

$$
(M-1)^{2} \geq M^{2}-2 M+1-2 M+4=1+(M-2)^{2} .
$$

Assim sendo tem-se como esperado $\left(1+(M-2)^{2}\right)^{1 / 2} \leq M-1 . \quad$ *

Passamos agora a mostrar que existe uma vizinhança de $V$ tal que todo operador $T \in \mathcal{L}\left(X, X^{\prime} \times \mathbb{R}\right)$ à ela pertencente constitui um isomorfismo sobre sua imagem que não atinge a respectiva norma em algum ponto de $C$.

Mostremos inicialmente que $\inf _{x \in \mathcal{S}_{X}(\|\cdot\|)}\|V x\|>0$. De fato, supondo o contrário existe uma sequência $\left(x_{n}\right)_{n \geq 1} \subset \mathcal{S}_{X}(\|\cdot\|)$ tal que $\left\|V\left(x_{n}\right)\right\| \stackrel{n \rightarrow \infty}{\longrightarrow} 0$. Porém

$$
\left\|V\left(x_{n}\right)\right\|^{2}=\left\|\left(x_{n}, M \cdot f\left(x_{n}\right)\right)\right\|^{2}=\left\|\mid x_{n}\right\|^{2}+\left(M \cdot f\left(x_{n}\right)\right)^{2}
$$

garantiria que $\left\|\mid x_{n}\right\|^{2} \stackrel{n \rightarrow \infty}{\longrightarrow} 0$, absurdo.

Considere portanto $\varepsilon=\inf _{x \in \mathcal{S}_{X}(\|\cdot \cdot\|)}\|V x\|>0$. Vamos mostrar que para $0<\varepsilon^{\prime}<\min \{\varepsilon, 1 / c, 1 / 3\}$ (onde $c>0$ é a constante presente na expressão (3.18)), todo $T \in \mathcal{L}(X, Y)$ tal que $\|T-V\|<\varepsilon^{\prime}$ constitui um isomorfismo sobre sua imagem que não atinge a respectiva norma em algum ponto de $C$.

a) $T$ é injetivo.

Supondo por absurdo a existência de $\xi \in \mathcal{S}_{X}(\|\cdot\|)$ tal que $\|T \xi\|=0$ teríamos

$$
\varepsilon \leq\|V \xi\|=\|V \xi-T \xi\| \leq\|V-T\|<\varepsilon,
$$

chegando na contradição esperada. 
b) $T$ constitui um isomorfismo sobre sua imagem.

Mostraremos que a aplicaçào $T^{-1}: T\left(X^{\prime}\right) \rightarrow X^{\prime}$ é limitada.

Dado qualquer $y \in T\left(X^{\prime}\right)$. tome $x_{y} \in X$ tal que $T\left(x_{y}\right)=y$. Temos

$$
\begin{aligned}
\|y\| & =\left\|T x_{y}\right\|=\left\|V x_{y}-\left(V x_{y}-T x_{y}\right)\right\| \geq\left\|V x_{y}\right\|-\varepsilon^{\prime}\left\|x_{y}\right\|= \\
& =\left\|\left(x_{y}, M \cdot f\left(x_{y}\right)\right)\right\|-\varepsilon^{\prime}\left\|x_{y}\right\| \geq\left\|x_{y}\right\| \mid-\varepsilon^{\prime} \cdot\left\|x_{y}\right\|= \\
& =\left\|x_{y}\right\|-\varepsilon^{\prime} \cdot c \cdot\left\|x_{y}\right\|=\left\|x_{y}\right\| \cdot\left(1-\varepsilon^{\prime} \cdot c\right) .
\end{aligned}
$$

Portanto

$$
\left\|T^{-1}(y)\right\|=\left\|x_{y}\right\| \leq \frac{1}{\left(1-\varepsilon^{\prime} \cdot c\right)}\|y\|
$$

e segue o desejado.

c) $T$ não atinge sua norma em algum ponto de $C$.

Uma vez que $M>2 / 3$, segue que:

- $\|T\| \geq M-1 / 3$.

De fato, uma vez que $\|V\|-\|T\| \leq\|V-T\| \leq \varepsilon^{\prime}$ e $\|V\| \geq M$, tem-se

$$
\|T\| \geq\|V\|-\|T-V\| \geq M-\varepsilon^{\prime} \geq M-1 / 3,
$$

como desejado.

- $\|T x\| \leq M-2 / 3, \forall x \in C$.

De fato, usando agora $\|T\|-\|V\| \leq\|T-V\|$ e $\|V x\| \leq M-1$ para todo $x \in C$ temos, para qualquer ponto $x$ fixado em $C$, que

$$
\begin{aligned}
\|T x\| & \leq\|V x\|+\|T x-V x\| \leq(M-1)+\|T-V\|< \\
& <(M-1)+1 / 3=M-2 / 3,
\end{aligned}
$$

novamente como queríamos mostrar. 
Podemos portanto concluir o desejado, bastando observar que, para qualquer ponto $x$ pertencente à $C$, temos

$$
\|T x\| \leq M-2 / 3<M-1 / 3 \leq\|T\|
$$

e assim sendo a norma de $T$ não pode ser atingida em pontos de $C$.

Observe que até aqui pudemos provar a existência de uma vizinhança $\Omega_{V}$ do operador $V$ em $\mathcal{L}(X, Y)$ constituida por elementos que não atingem suas respectivas normas em pontos de $C$.

Uma vez que $X$ tem por hipótese a propriedade $A$, segue que deve existir algum ponto $x_{p} \in \mathcal{S}_{X}(\|\cdot\|)$ tal que

$$
W\left(x_{p}\right)=\|W\| \text { para algum } W \in \Omega_{V} .
$$

Porém a proposição (3.34), aliada à proposiçào (3.39), garante-nos que $x_{p}$ é ponto [fortemente] exposto de $\mathcal{B}_{X}(\|\cdot\|) \mathrm{e}$, portanto, elemento de $C$. A contradição com o penúltimo parágrafo encerra a demostração.

\section{Corolário 3.43}

A bola unitária $\mathcal{B}_{X}(\|\cdot\|)$ de um espaço de Banach separável e reflexivo $(X,\|\cdot\|)$ coincide com a envoltória convexa fechada de seus pontos fortemente expostos.

Prova: Do corolário (3.7) segue que $X$ tem a propriedade $A$. Uma vez que todo espaço de Banach separável é isomorfo a um espaço de Banach localmente uniformemente convexo ([Kadec]) segue do teorema a conclusão desejada. 
Apresentamos a seguir um resultado de renormagem que terá importância na proposição que o sucede.

Teorema 3.44 ("the Day's norm" restrita à $c_{0}$ )

A aplicação $\|\cdot\|: c_{0} \rightarrow \mathbb{R}$ dada por

$\|x\|=\sup _{n \in \mathbb{N}}\left\{\left(\sum_{j=1}^{n} \frac{x_{k_{j}}^{2}}{4^{j}}\right)^{1 / 2}:\left(k_{1}, \ldots, k_{n}\right) \subset \mathbb{N}^{n} \epsilon^{\prime} n\right.$-upla de números distintos $\}$

constitui uma norma localmente uniformemente convera em $c_{0}$ equivalente à usual.

Prova: Vide por exemplo [DvlGdfr], Teor (7.3). 69.

\section{Proposição 3.45}

Existe espaço de Banach $(X,\|\cdot\|)$ tal que $\mathcal{N} \mathcal{A}(X, X)$ não é denso em $\mathcal{L}(X, X)$.

Prova: Considere $Y=c_{0}$ munido da norma usual e $Z$ um espaço de Banach estritamente convexo e isomorfo a $c_{0}$ (cuja existência está garantida pelo teorema (3.44) associado à observação (3.21)).

Definindo $X=Y \times Z$ e uma norma $\|\cdot\|$ em $X$ dada por $\|(y, z)\|=$ $\max \{\|y\|,\|z\|\}$, passemos a mostrar que o espaço de Banach $(X,\|\cdot\|)$ assim definido satisfaz o desejado.

Sendo o isomorfismo existente entre $Y$ e $Z$ dado pelo operador $T_{0}$ que, sem perda de generalidade, podemos assumir satisfazer $\left\|T_{0}\right\| \leq 1$, defino $T \in \mathcal{L}(X, X)$ por

$$
(y, z) \in X=Y \times Z \stackrel{T}{\longmapsto}\left(0, T_{0} y\right) .
$$

Note que $T$ é obviamente linear e também limitada uma vez que

$$
\begin{aligned}
\|T(y, z)\| & =\left\|\left(0, T_{0} y\right)\right\|=\left\|T_{0} y\right\| \leq\left\|T_{0}\right\|\|y\| \leq \\
& \leq\|y\| \leq \max \{\|y\|,\|z\|\}=\|(y, z)\|
\end{aligned}
$$

e portanto $\|T\| \leq 1$. 
Sendo $T_{0}$ um homeomorfismo linear, $T_{0}^{-1}$ é linear e limitada. Existe portanto uma constante $c>0$ tal que

$$
\left\|T_{0}^{-1} z\right\| \leq c \cdot\|z\|, \quad \forall z \in Z
$$

e, pela bijetividade de $T_{0}$ segue que, para cada $\approx \in Z$ existe um único $y=y(z) \in Y$ tal que $T_{0}(y)=z$. Entào

$$
\|y\|=\left\|T_{0}^{-1}\left(T_{0} y\right)\right\| \leq c \cdot\left\|T_{0} y\right\|
$$

e, assim sendo,

$$
\left\|T_{0} y\right\| \geq c^{-1} \cdot\|y\|, \quad \forall y \in Y .
$$

Tomando $0<\varepsilon \leq 1 /(2 c)$ garante-se que

$$
\left\|T_{0} y\right\| \geq 2 \varepsilon\|y\|, \quad \forall y \in Y .
$$

Suponha, por absurdo, que houvesse $\hat{T} \in \mathcal{L}\left(X^{\prime}, X^{r}\right)$ tal que $\|\hat{T}-T\|<\varepsilon$ e $\|\hat{T}\|=\left\|\hat{T}\left(y_{0}, z_{0}\right)\right\|$ para algum $\left(y_{0}, z_{0}\right) \in \mathcal{S}_{X}(\|\cdot\|)$. Fazendo então $\hat{T}\left(y_{0}, z_{0}\right)=(u, v) \in Y \times Z$, teremos $\|u\|<\varepsilon$ uma vez que

$$
\|u\| \leq\left\|(u, v)-\left(0, T_{0} y_{0}\right)\right\|=\left\|\hat{T}\left(y_{0}, z_{0}\right)-T\left(y_{0}, z_{0}\right)\right\|<\varepsilon .
$$

Provemos agora que $\|\hat{T}\|>\varepsilon$.

Caso $T_{0}^{-1}\left(z_{0}\right)=0$, então $z_{0}=0$ e portanto $\left\|y_{0}\right\|=1$. Da desigualdade triangular, definição da aplicação $T$ e de (3.19) segue que

$$
\begin{aligned}
\left\|T\left(y_{0}, 0\right)-\hat{T}\left(y_{0}, 0\right)\right\|+\left\|\hat{T}\left(y_{0}, 0\right)\right\| & \geq\left\|T\left(y_{0}, 0\right)\right\|=\left\|\left(0, T_{0} y_{0}\right)\right\|= \\
& =\left\|T_{0} y_{0}\right\| \geq 2 \varepsilon \cdot\left\|y_{0}\right\|=2 \varepsilon,
\end{aligned}
$$

donde podemos escrever

$$
\begin{aligned}
\|\hat{T}\| & \geq\left\|\hat{T}\left(y_{0}, 0\right)\right\| \geq 2 \varepsilon-\left\|(T-\hat{T})\left(y_{0}, 0\right)\right\| \geq \\
& \geq 2 \varepsilon-\|T-\hat{T}\| \cdot\left\|\left(y_{0}, 0\right)\right\|>2 \varepsilon-\varepsilon=\varepsilon,
\end{aligned}
$$

como almejado.

Caso contrário podemos considerar o vetor $\eta=T_{0}^{-1}\left(z_{0}\right) /\left\|T_{0}^{-1}\left(z_{0}\right)\right\|$. Da desigualdade triangular, definição da aplicação $T$ e de (3.19) segue que

$$
\|T(\eta, 0)-\hat{T}(\eta, 0)\|+\|\hat{T}(\eta, 0)\| \geq\|T(\eta, 0)\|=\left\|\left(0, T_{0} \eta\right)\right\|=\left\|T_{0} \eta\right\| \geq 2 \varepsilon
$$


e podemos escrever

$$
\|\hat{T}\| \geq\|\hat{T}(\eta, 0)\| \geq 2 \varepsilon-\|(T-\hat{T})(\eta, 0)\| \geq 2 \varepsilon-\|T-\hat{T}\| \cdot\|(\eta, 0)\|>\varepsilon,
$$

como desejado.

Uma vez que $\|u\|<\varepsilon$ e $\|\hat{T}\|>\varepsilon$ temos entào

$$
\|u\|<\|\hat{T}\|=\left\|\hat{T}\left(y_{0}, \tilde{z}\right)\right\|=\|(u, v)\|=\max \{\|u\|,\|v\|\}=\|v\| .
$$

Observando que a bola unitária de $c_{0}=Y$ não admite pontos extremais (exemplo (3.25)) garantimos, em particular, que $y_{0}$ pertence a algum segmento de reta aberto ${ }^{9}$ contido na bola unitária $\mathcal{B}_{Y}(\|\cdot\|)$. Este argumento geométrico nos permite garantir a existência de um ponto $y_{1} \in Y \backslash\left\{y_{0}\right\}$ tal que

$$
0<\left\|y_{1}\right\| \leq 1 \quad \text { e } \quad\left\|y_{1}+y_{0}\right\|=\left\|-y_{1}+y_{0}\right\| \leq 1 .
$$

Usaremos a partir daqui o símbolo " \pm " para indicar a validade, para ambos sinais, da expressão que o contém.

De $\left\|\left(y_{0} \pm y_{1}, z_{0}\right)\right\|=\max \left\{\left\|y_{0} \pm y_{1}\right\|,\left\|z_{0}\right\|\right\} \leq 1$ teremos

$$
\left\|\hat{T}\left(y_{0}, z_{0}\right) \pm \hat{T}\left(y_{1}, 0\right)\right\|=\left\|\hat{T}\left(y_{0} \pm y_{1}, z_{0}\right)\right\| \leq\|\hat{T}\| .
$$

Se existe $y_{2} \in Y$ tal que $\hat{T}\left(y_{1}, 0\right)=\left(y_{2}, 0\right)$, como $\left\|y_{1}\right\| \leq 1$ segue então

$$
\begin{aligned}
2 \varepsilon\left\|y_{1}\right\| & \leq\left\|T_{0} y_{1}\right\| \leq\left\|\left(y_{2},-T_{0} y_{1}\right)\right\|=\left\|\left(y_{2}, 0\right)-\left(0, T_{0} y_{1}\right)\right\|= \\
& =\left\|\hat{T}\left(y_{1}, 0\right)-T\left(y_{1}, 0\right)\right\| \leq\|\hat{T}-T\| \cdot\left\|\left(y_{1}, 0\right)\right\|<\varepsilon\left\|y_{1}\right\|,
\end{aligned}
$$

que é a contradiçâo esperada. Vejamos agora a prova da existência de um elemento $y_{2} \in Y$ nesta condição.

Fazendo $\hat{T}\left(y_{1}, 0\right)=\left(y_{2}, z_{2}\right) \in Y \times Z$ tem-se, da expressão (3.21), que $\|V\|=\|\hat{T}\| \geq\left\|\hat{T}\left(y_{0}, z_{0}\right) \pm \hat{T}\left(y_{1}, 0\right)\right\|=\left\|(u, v) \pm\left(y_{2}, z_{2}\right)\right\|=\left\|\left(u \pm y_{2}, v \pm z_{2}\right)\right\|$ e portanto

$$
\max \left\{\left\|u \pm y_{2}\right\|,\left\|v \pm z_{2}\right\|\right\} \leq\|v\| \Rightarrow\left\|v \pm z_{2}\right\| \leq\|v\| .
$$

\footnotetext{
${ }^{9}$ No sentido de considerá-lo sem suas extremidades. [Day] define um ponto nestas condições como um "passing point" da bola.
} 
Segue então que

$2\|v\|=\left\|v+z_{2}+v-z_{2}\right\| \leq\left\|v+z_{2}\right\|+\left\|v-z_{2}\right\| \leq\|v\|+\|v\|=2\|v\|$

garantindo portanto $\left\|v+z_{2}\right\|+\left\|v-z_{2}\right\|=2\|v\|$.

Porém, uma vez que $p=\left(v+z_{2}\right) / 2$ e $q=\left(v-z_{2}\right) / 2$ são tais que $\|p\|+\|q\|=\|v\|=\|p+q\|$, valendo-nos da proposição (3.10. e)) podemos afirmar ${ }^{10}$ que existe $\lambda \geq 0$ tal que $q=\lambda p$. Logo $|\lambda|=1$ uma vez que $\left\|v+z_{2}\right\|=\|v\|=\left\|v-z_{2}\right\|$ implica em $\|p\|=\|q\|$.

Caso $\lambda=-1$ tem-se $v=0$, absurdo pois $\|v\|=\|\hat{T}\|>\Xi$. Conclui-se então que $\lambda=1$ e assim $p=q$ e $z_{2}=0$.

${ }^{10}$ Temos $p$ ou $q$ não nulo; sem perda de generalidade consideremos $p$ nesta condição. 


\section{Capítulo 4}

\section{O espaço de Gowers e um contra-exemplo do Bishop-Phelps para bilineares}

\subsection{Introdução}

Algumas questões propostas por Lindenstrauss e relacionadas com a propriedade $B$ ficaram muito tempo sem resposta. Demoraram quase trinta anos para que W.T.Gowers utilizasse um espaço de Banach ([Gowers]) que fosse capaz de responder uma delas: o espaço $l^{p}(1<p<\infty)$ tem a propriedade $B$ ?

Estudaremos aqui interessantes propriedades do espaço de Gowers que, além de responder (negativamente) a questão, são capazes de proporcionar vários outros resultados associados ao mesmo tema, muitos deles desenvolvidos por Maria D.Acosta, Franscisco J.Aguirre e Rafael Payá ([A.A.P.1] e [A.A.P.2]).

No final desta dissertação apresentaremos uma interessante aplicação das técnicas desenvolvidas até então: a prova de que não existe, para um espaço de Banach $(X,\|\cdot\|)$ qualquer, um "teorema de Bishop-Phelps bilinear", considerando uma generalização bastante natural apresentada pelos três matemáticos citados no final do parágrafo anterior. 


\subsection{O espaço de Gowers e algumas aplicações}

\section{Definição 4.1}

Dada uma sequência $x=\left(x_{i}\right)_{i \geq 1}$ de reais, considere para cada $n \geq 1$ a soma parcial da série harmônica $H_{n}=\sum_{k=1}^{n} k^{-1}, J \subset \mathbb{N}$ qualquer subconjunto de $\mathbb{N}$ com cardinalidade $|J|=n$ e a aplicaçâo

$$
\varphi_{n}(x)=\frac{1}{H_{n}} \sup _{|J|=n} \sum_{j \in J}\left|x_{j}\right| \text {. }
$$

$O$ espaço $G=\left\{x=\left(x_{i}\right)_{i \geq 1}: \varphi_{n}(x) \stackrel{n \rightarrow \infty}{\longrightarrow} 0\right\}$ munido das operaçôes usuais nas sequências constitui um espaço vetorial.

Proposição 4.2 Todo elemento de $G$ é uma sequência que converge para zero.

Prova: Suponhamos por absurdo a existência de $x=\left(x_{i}\right)_{i \geq 1} \in G$ e $\varepsilon>0$ tais que $\left|x_{i_{k}}\right| \geq \varepsilon, \forall k \geq 1$. Para todo $n \geq 1$ temos então

$$
\varphi_{n}(x)=\frac{1}{H_{n}} \sup _{|J|=n} \sum_{j \in J}\left|x_{j}\right| \geq \frac{1}{H_{n}} \sum_{k=1}^{n}\left|x_{i_{k}}\right| \geq \frac{1}{H_{n}}(n \varepsilon) \geq \frac{n \varepsilon}{n}=\varepsilon,
$$

valendo a última desigualdade uma vez que $H_{n} \leq n, \forall n \geq 1$.

Portanto $\varphi_{n}(x)$ não converge para zero, contradizendo o fato de $x \in G$.

\section{Proposição 4.3}

A aplicação $\|\cdot\|: G \rightarrow \mathbb{R}$ dada por

$$
\|x\|=\sup _{n \in \mathbb{N}} \varphi_{n}(x)
$$

constitui uma norma em $G$ que o faz completo. Além disso, o espaço de Banach $(G,\|\cdot\|)$ (conhecido como o "espaço de Gowers") admite o conjunto ${ }^{1}$ $\left\{e_{n}\right\}_{n \geq 1}$ como base de Schauder.

\footnotetext{
${ }^{1}$ onde $e_{k}=(0,0, \ldots, 0,1,0, \ldots)$ com o valor 1 na $k \underline{a}$ coordenada.
} 
Prova: Da demonstração que a aplicação $\|\cdot\|$ assim definida é norma em $G$, faremos apenas a desigualdade triangular. Dados quaisquer $x, y \in G$, da definiçào de $\varphi_{n}$ segue de imediato que $\varphi_{n}(x+y) \leq \varphi_{n}(x)+\varphi_{n}(y)$ $(\forall n \geq 1)$ e como $\|x+y\|=\sup _{n \in \mathbb{N}} \varphi_{n}(x+y)$, segue que:

$$
\begin{aligned}
\|x+y\| & \leq \sup _{n \in \mathbb{N}}\left(\varphi_{n}(x)+\varphi_{n}(y)\right) \\
& \leq \sup _{n \in \mathbb{N}} \varphi_{n}(x)+\sup _{n \in \mathbb{N}} \varphi_{n}(y)=\|x\|+\|y\|
\end{aligned}
$$

como desejado.

Para justificar que $(G,\|\cdot\|)$ é Banach basta observar que, valendo-se do teorema (1.37) aplicado ao caso particular da sequência $w=\left(w_{n}=1 / n\right)_{n \geq 1}$, podemos facilmente notar que o espaço de Gowers identifica-se (na notação do referido teorema) com o espaço de Banach $\left(d_{*}(\{1 / n\}, 1),\|\cdot\|\right)$ munido da norma lá apresentada e o mesmo teorema nos garante $\left\{\epsilon_{n}\right\}_{n \geq 1}$ como base de Schauder para $(G,\|\cdot\|)$.

Entenderemos a partir daqui a "base de Schauder associada ao espaço de Gowers" como sendo a base $\left(e_{n}\right)_{n \geq 1}$ aqui utilizada.

Passamos a provar um resultado elementar que será de grande valia no lema que o sucede.

\section{Lema 4.4}

Dado qualquer $n \geq 1$, sejam $a_{1} \geq a_{2} \geq \ldots \geq a_{n} \geq 0$ números reais tais que

$$
\sum_{i=1}^{m} a_{i} \leq \sum_{i=1}^{m} i^{-1} \quad, \forall 1 \leq m \leq n .
$$

Então dado qualquer $1<p<\infty$, temos

$$
\sum_{i=1}^{n} a_{i}^{p} \leq \sum_{i=1}^{n} i^{-p} .
$$


Prova: Fixados $1<p<\infty$ e $n \geq 1$, como o conjunto $\Omega_{n}=\left\{\left(x_{1}, x_{2}, \ldots, x_{n}\right) \in \mathbb{R}^{n}: x_{1} \geq x_{2} \geq \cdots \geq x_{n} \geq 0 \mathrm{e} \sum_{i=1}^{n} x_{i} \leq \sum_{i=1}^{n} i^{-1}\right\}$ é um compacto do $\mathbb{R}^{n}$ e a aplicação $f: \Omega_{n} \rightarrow \mathbb{R}$ dada por

$$
f\left(x_{1}, x_{2}, \ldots, x_{n}\right)=\sum_{i=1}^{n} x_{i}^{p}
$$

é contínua, $f\left(\Omega_{n}\right)$ admite máximo. Assim sendo, podemos afirmar que existem $b_{1} \geq b_{2} \geq \cdots \geq b_{n} \geq 0$ tais que

$$
\sum_{i=1}^{m} b_{i} \leq \sum_{i=1}^{m} i^{-1}, \forall 1 \leq m \leq n
$$

e, consequentemente, para qualquer $1<p<\infty$ teremos

$$
\sum_{i=1}^{n} b_{i}^{p} \geq \sum_{i=1}^{n} a_{i}^{p} .
$$

Separemos a continuação em algumas etapas.

a) Supondo $b_{i}=i^{-1}$ para todo $1 \leq i \leq n$, temos para qualquer destes índices que $b_{i}^{p}=i^{-p}$ e portanto

$$
\sum_{i=1}^{n} a_{i}^{p} \leq \sum_{i=1}^{n} b_{i}^{p} \leq \sum_{i=1}^{n} i^{-p},
$$

satisfazendo a tese.

b) Existe um índice $1 \leq j \leq n$ mínimo para o qual $b_{j}<1 / j$.

Suponha que não houvesse $j$ nestas condições. Teríamos $b_{j} \geq 1 / j$ para todo $1 \leq j \leq n$ e então

$$
\sum_{i=1}^{m} i^{-1} \geq \sum_{i=1}^{m} b_{i} \geq \sum_{i=1}^{m} i^{-1}, \forall 1 \leq m \leq n,
$$

garantindo $\sum_{i=1}^{m} b_{i}=\sum_{i=1}^{m} i^{-1}$ e seguiria $b_{i}=i^{-1}$ para todo $1 \leq i \leq n$, recaindo no item anterior. 
c) Tem-se em realidade $1 \leq j \leq n-1$.

Suponhamos que tenhamos $j=n$. Da minimalidade associada à $j$ conclui-se, por argumentos idênticos ao item anterior, que $b_{i}=i^{-1}$ para todo $1 \leq i \leq n-1$. Usando agora a maximalidade de $\sum_{i=1}^{n} b_{i}^{p}$ aliada ao fato de $\sum_{i=1}^{n} b_{i} \leq \sum_{i=1}^{n} i^{-1}$, pode-se afirmar que $b_{n}=n^{-1}$ e novamente recai-se no primeiro item.

d) Existe $1 \leq k \leq n$ máximo para o qual $\sum_{i=1}^{k} b_{i}=\sum_{i=1}^{k} i^{-1}$.

Supondo por absurdo que não, teremos de $\sum_{i=1}^{m} b_{i} \leq \sum_{i=1}^{m} i^{-1}$ (para qualquer $1 \leq m \leq n$ ) que

$$
\sum_{i=1}^{m} b_{i}<\sum_{i=1}^{m} i^{-1} \quad, \forall 1 \leq m \leq n .
$$

Para $m=1$ a expressão garante que $1>b_{1}$ (portanto $j=1$ ) e da existência de $\varepsilon>0$ tal que

$$
1>b_{1}+\varepsilon>b_{1} \geq b_{2} \geq \cdots \geq b_{n} \geq 0
$$

posso definir $c_{1}, c_{2}, \ldots, c_{n}$ dados por

$$
c_{i}= \begin{cases}b_{i}+\varepsilon & \text { se } i=1 \\ b_{i} & \text { se } 1<i \leq n\end{cases}
$$

que satisfazem as hipóteses do enunciado e são tais que $\sum_{i=1}^{n} c_{i}^{p}>$ $\sum_{i=1}^{n} b_{i}^{p}$, contradizendo a maximalidade da soma $\sum_{i=1}^{n} b_{i}^{p}$.

e) Tem-se em realidade $k>j$.

Caso $k=n$ então $j<k$ pois $j \neq n$. Do contrário, supondo por absurdo que $1 \leq k \leq j \leq n-1$, da minimalidade associada à $j$ temos $b_{k}=1 / k$ e da maximalidade associada à $k$ podemos escrever

$$
\sum_{i=1}^{k} b_{i}=\sum_{i=1}^{k} i^{-1} \text { e } \sum_{i=1}^{k+1} b_{i}<\sum_{i=1}^{k+1} i^{-1}
$$

e portanto certamente $b_{k+1}<1 /(k+1)$. Segue então que $b_{1}=1>b_{2}=\frac{1}{2}>\cdots>b_{k}=\frac{1}{k}>\frac{1}{k+1}>b_{k+1} \geq b_{k+2} \geq \cdots \geq b_{n} \geq 0$ 
e constatando a existência de $\varepsilon>0$ tal que $b_{k}>b_{k+1}+\varepsilon>b_{k+1}$ podemos proceder como no item anterior criando $\left(c_{i}\right)_{i \geq 1}^{n}$ que contradiz a maximalidade de $\sum_{i=1}^{n} b_{i}^{p}$.

Observe que dos itens provados podemos concluir que

- Existe $\varepsilon_{1}>0$ tal que $b_{k}>b_{k}-\varepsilon_{1}>b_{k+1}$ para algum $(j+1) \leq k \leq$ $(n-1)$.

- Caso $j=1$ existe $\varepsilon_{2}>0$ tal que $1>b_{1}+\varepsilon>b_{1} \quad$ (feito dentro do item d)) .

- Caso $j>1$ existe $\varepsilon_{3}>0$ tal que $b_{j-1}>b_{j}+\varepsilon>b_{j}$ (procedendo-se como em e)) .

Tomando $\varepsilon=\min \left\{\varepsilon_{1}, \varepsilon_{2}, \varepsilon_{3}\right\}>0$, podemos trocar $b_{j}$ por $b_{j}+\varepsilon \mathrm{e}$ $b_{k}$ por $b_{k}-\varepsilon$ sem violar quaisquer das hipóteses do enunciado e ainda continuar contradizendo a maximalidade de $\sum_{i=1}^{n} b_{i}^{p}$. De fato, valendo-nos do binômio de Newton podemos afirmar que

$\left(b_{j}+\varepsilon\right)^{p}+\left(b_{k}-\varepsilon\right)^{p}=b_{j}^{p}+b_{k}^{p}+p \cdot\left(b_{j}^{p-1}-b_{k}^{p-1}\right) \cdot \varepsilon+\frac{p(p-1)}{2} \cdot\left(b_{j}^{p-2}+b_{k}^{p-2}\right) \cdot \varepsilon^{2}+o\left(\varepsilon^{2}\right)$

com $o\left(\varepsilon^{2}\right)$ substituindo os termos que possuem, para $j \geq 3$, $\varepsilon^{j}$ como fator.

Como $j<k$ segue que $b_{j} \geq b_{k}$ e, portanto, para $\varepsilon>0$ suficientemente pequeno, a soma dos últimos três termos será positiva. Assim sendo,

$$
\left(b_{j}+\varepsilon\right)^{p}+\left(b_{k}-\varepsilon\right)^{p}>b_{j}^{p}+b_{k}^{p}
$$

e a maximalidade de $\sum_{i=1}^{n} b_{i}^{p}$ ficou de fato comprometida.

Finalmente, notando que tudo o que fizemos mostra que $\sum_{i=1}^{n} b_{i}^{p}$ máxima implica (com as demais restriçôes) em valer $b_{i}=i^{-1}$ para todo $1 \leq i \leq n$, segue no caso geral que temos $\sum_{i=1}^{n} i^{-p}$ como majorante, exatamente o que queríamos demonstrar. 


\section{Lema 4.5}

Considere $(G,\|\cdot\|)$ o espaço de Gowers e $1<p<\infty$ fixado. A aplicação $T: G \rightarrow l^{p}$ definida por

$$
T(x)=\left(x_{1}, x_{2}, \ldots\right)
$$

onde $x=\left(x_{i}\right)_{i \geq 1} \in G$ é linear, injetiva e limitada. Consequentemente, podemos entender $T$ como sendo a imersão de $G \mathrm{em}^{p}$.

Prova: Iniciamos por garantir que $T(G) \subset l^{p}$. Dado qualquer $a=\left(a_{i}\right)_{i \geq 1} \in G$, suponhamos que $a \in \mathcal{S}_{G}(\|\cdot\|)$. Neste caso como a série $\sum_{i \geq 1} i^{-p}$ converge para todo $1<p<\infty$, basta mostrarmos que

$$
\|T a\| \leq\left(\sum_{i \geq 1} i^{-p}\right)^{1 / p} .
$$

Com efeito, uma vez que

$$
1=\|a\|=\sup _{n \in \mathbb{N}} \varphi_{n}(a)=\sup _{n \in \mathbb{N}}\left(\frac{1}{H_{n}} \sup _{|J|=n} \sum_{j \in J}\left|a_{j}\right|\right)
$$

podemos, para qualquer $n \geq 1$ fixado, assumir que os $n$ primeiros termos da sequência $\left(a_{i}\right)_{i \geq 1}$ são constituidos por reais não negativos ordenados de forma não crescente e satisfazendo

$$
\sum_{i=1}^{m} a_{i} \leq H_{m}=\sum_{i=1}^{m} i^{-1}
$$

para todo $1 \leq m \leq n$. Valendo-nos do lema (4.4) podemos garantir que $\sum_{i=1}^{n} a_{i}^{p} \leq \sum_{i=1}^{n} i^{-p}$. Fazendo $n \rightarrow \infty$ teremos

$$
\|T a\|^{p}=\left\|\left(a_{1}, a_{2}, \ldots\right)\right\|^{p}=\sum_{i \geq 1}\left|a_{i}\right|^{p} \leq \sum_{i \geq 1} i^{-p}
$$

donde segue o desejado. Como para todo $x \in G \backslash \mathcal{S}_{G}(\|\cdot\|)$ tem-se $x=\mathbf{0}$ ou $x /\|x\| \in \mathcal{S}_{G}(\|\cdot\|)$, no primeiro caso $T(\mathbf{0})=0 \in l^{p}$ e no segundo

$$
\frac{1}{\|x\|}\|T(x)\|=\left\|T\left(\frac{x}{\|x\|}\right)\right\| \leq\left(\sum_{i \geq 1} i^{-p}\right)^{1 / p}
$$


e portanto $T(G) \subset l^{p}$ como almejado.

A injetividade de $T$ é imediata e sua limitação é decorrente das mesmas desigualdades obtidas.

Observação 4.6 A imersão $T: G \rightarrow l^{p}$ não é compacta.

Supondo o contrário, o teorema (1.20) nos garantiria que toda sequência definida no compacto $\overline{T\left(\mathcal{B}_{G}(\|\cdot\|)\right)} \subset l^{p}$ admitiria uma subsequência convergente na norma usual do $l^{p}$. Tomando em particular a base de Schauder $\left(e_{i}\right)_{i \geq 1}$ associada ao espaço de Gowers, como $T\left(e_{i}\right)=\epsilon_{i}$ para todo $i \geq 1$, segue que tal sequência deveria admitir uma subsequência convergente. Isto não é possível uma vez que para quaisquer índices distintos $j, k$ tem-se $\left\|e_{j}-e_{k}\right\|_{p}=\sqrt[p]{2}$.

\section{Lema 4.7}

Seja $(G,\|\cdot\|)$ o espaço de Gowers $\epsilon\left(e_{i}\right)_{i \geq 1}$ a base de Schauder associada. Dado qualquer $x \in \mathcal{S}_{G}(\|\cdot\|)$, existem $N_{0} \geq 1 \epsilon \delta>0$ tais que $\left\|x+\lambda \cdot e_{i}\right\|=1$, para quaisquer $i \geq N_{0}$ e $\lambda$ real tal que $|\lambda| \leq \delta$. que:

Prova: Considerando $x=\left(x_{i}\right)_{i \geq 1} \in \mathcal{S}_{G}(\|\cdot\|)$ segue de $\hat{\varphi}_{n}(x) \stackrel{n \rightarrow \infty}{\longrightarrow} 0$

- Existe $N_{1} \geq 1$ tal que $\varphi_{n}(x)<1 / 2$, para todo $n \geq N_{1}$.

- Pela proposição (4.2) sabemos que $x_{i} \stackrel{i \rightarrow \infty}{\longrightarrow} 0$. Assim sendo, existe $N_{0} \geq N_{1}$ tal que

$$
\left|x_{i}\right| \leq \frac{1}{2 N_{1}} \quad, \forall i \geq N_{0} .
$$

Tomando $0<\delta \leq 1 /\left(2 N_{1}\right)$ e qualquer $i_{0} \geq N_{0}$, mostremos que para todo $\lambda \in \mathbb{R}$ tal que $|\lambda| \leq \delta$, o elemento $y=x+\lambda \cdot \epsilon_{i_{0}}$ satisfaz $\varphi_{n}(y) \leq 1$, para todo $n \geq 1$.

De fato, considerando $e_{i_{0}, j}$ a $j^{\underline{a}}$ coordenada de $e_{i_{0}}$, como

$$
\varphi_{n}\left(\epsilon_{i_{0}}\right)=\frac{1}{H_{n}} \sup _{|J|=n} \sum_{j \in J}\left|e_{i_{0}, j}\right| \leq 1
$$


e $\varphi_{n}\left(x+\lambda \cdot \epsilon_{i_{0}}\right) \leq \varphi_{n}(x)+\varphi_{n}\left(\lambda \cdot \epsilon_{i_{0}}\right)$ como decorrência do argumento dado na proposiçào (4.3). segue que para $n \geq N_{1}$ temos:

$$
\varphi_{n}(y) \leq \varphi_{n}(x)+|\lambda| \cdot \varphi_{n}\left(e_{i_{0}}\right)<\frac{1}{2}+|\lambda| \leq \frac{1}{2}+\delta \leq 1
$$

como desejado. Resta ainda analisar o caso $1 \leq n<N_{1}$. Tomando qualquer subconjunto $J \subset \mathbb{N}$ formado por $n$ elementos distintos, para garantir que $\varphi_{n}(y) \leq 1$ basta mostrar que $\sum_{k \in J}\left|y_{k}\right| \leq H_{n}$.

Supondo inicialmente que $i_{0} \in J$, de $y_{i_{0}}=x_{i_{0}}+\lambda$ e $y_{k}=x_{k}$ sempre que $k \neq i_{0}$, teremos

$$
\begin{aligned}
\sum_{k \in J}\left|y_{k}\right| & =\left|y_{i_{0}}\right|+\sum_{k \in J \backslash\left\{i_{0}\right\}}\left|y_{k}\right| \leq \delta+\left|x_{i_{0}}\right|+\sum_{k \in J \backslash\left\{i_{0}\right\}}\left|x_{k}\right| \leq \\
& \leq \frac{1}{2 N_{1}}+\frac{1}{2 N_{1}}+\sum_{k \in J \backslash\left\{i_{0}\right\}}\left|x_{k}\right|=\frac{1}{N_{1}}+\sum_{k \in J \backslash\left\{i_{0}\right\}}\left|x_{k}\right| .
\end{aligned}
$$

Como $1=\|x\|=\sup _{n \in \mathbb{N}}\left(1 / H_{n}\right) \sup _{|J|=n} \sum_{j \in J}\left|x_{j}\right|$, segue em particular que $\sum_{k \in J \backslash\left\{i_{0}\right\}}\left|x_{k}\right| \leq H_{n-1}$.

Levando-se em conta as últimas expressões e o fato de estarmos considerando $n<N_{1}$ teremos

$$
\sum_{k \in J}\left|y_{k}\right| \leq \frac{1}{N_{1}}+\sum_{k \in J \backslash\left\{i_{0}\right\}}\left|x_{k}\right| \leq \frac{1}{N_{1}}+H_{n-1}<\frac{1}{n}+H_{n-1}=H_{n},
$$

como desejado.

Caso $i_{0} \notin J$, como $J$ tem $n$ elementos segue de imediato o esperado:

$$
\sum_{k \in J}\left|y_{k}\right|=\sum_{k \in J}\left|x_{k}\right| \leq \sup _{|J|=n} \sum_{j \in J}\left|x_{j}\right| \leq H_{n},
$$

a última desigualdade obtida dois parágrafos atrás.

Finalmente podemos concluir que, para qualquer $i \geq N_{0}$,

$$
\|y\|=\left\|x+\lambda \cdot e_{i}\right\|=\sup _{n \geq 1} \varphi_{n}\left(x+\lambda e_{i}\right) \leq 1 .
$$

Para mostrar que $\|y\|=1$, suponhamos por absurdo que o supremo acima seja menor que $1 \mathrm{e}$, portanto, existe $\varepsilon_{0}>0$ tal que $\varphi_{n}\left(x+\lambda \epsilon_{i}\right) \leq$ $1-\varepsilon_{0}$, para qualquer $n \geq 1$ e $i \geq N_{0}$. Tomando $\lambda=0$ teríamos $\|x\|<1$, impossível. 


\section{Corolário 4.8}

Seja $(G,\|\cdot\|)$ o espaço de Gowers. Sua bola unitária $\mathcal{B}_{G}(\|\cdot\|)$ nào admite pontos extremais.

Prova: Basta considerarmos os pontos pertencentes à esfera $\mathcal{S}_{G}(\|\cdot\|)$. Dado qualquer $x \in \mathcal{S}_{G}(\|\cdot\|)$, o lema anterior nos garante a existência de $i_{0} \geq 1$ e $\delta>0$ para os quais $\left\|x \pm \delta \cdot \epsilon_{i_{0}}\right\|=1$ e, portanto, o ponto $x$ pertence ao segmento de reta aberto

]$x-\delta \cdot e_{i_{0}}, x+\delta \cdot e_{i_{0}}\left[=\left\{z \in G: z=x+\lambda \cdot e_{i_{0}},-\delta<\lambda<\delta\right\} \subset \mathcal{S}_{G}(\|\cdot\|)\right.$

$\mathrm{e}$, assim sendo, $x$ não é extremal.

\section{Proposição 4.9 (Gowers)}

Considere $1<p<\infty$ e $\left(e_{i}\right)_{i \geq 1}$, a base de Schauder do espaço de Gowers $(G,\|\cdot\|)$. Para cada $T \in \mathcal{N}^{-} \mathcal{A}\left(G, l^{p}\right)$ existe um indice $N_{T} \geq 1$ tal que

$$
T\left(e_{i}\right)=\mathbf{0}, \forall i \geq N_{T}
$$

Prova: Seja $a=\left(a_{i}\right)_{i \geq 1} \in \mathcal{S}_{G}(\|\cdot\|)$ um ponto da esfera tal que $\|T a\|=\|T\|$. Pelo lema (4.7), existem $N_{T} \geq 1$ e $0<\delta<1$ para os quais

$$
\left\|a+\delta \cdot e_{i}\right\|=\left\|a-\delta \cdot e_{i}\right\|=1, \forall i \geq N_{T} .
$$

Nestas mesmas condições temos então

$$
\left\|T a+\delta \cdot T \epsilon_{i}\right\| \leq\|T\|=\|T a\| \quad \text { e }\left\|T a-\delta \cdot T e_{i}\right\| \leq\|T\|=\|T a\| .
$$

Uma vez que o espaço $l^{p}$ é estritamente convexo (exemplo (3.12)) e $\delta \neq 0$, a técnica presente na proposição (3.45) nos permite concluir o desejado.

\section{Corolário 4.10}

$O$ espaço lp $^{p}(1<p<\infty)$ não tem a propriedade $B$.

Prova: Da proposição (4.9) podemos concluir que todo $T \in \mathcal{N} \mathcal{A}\left(G, l^{p}\right)$ tem posto finito ${ }^{2}$ e, pelo teorema (1.21), é compacto. Assim todo operador

2i.e, $\operatorname{dim}(T(G))<\infty$. 
presente no fecho de $\mathcal{N} \mathcal{A}\left(G, l^{p}\right)$ é compacto e a observação (4.6) nos permite concluir que tal fecho é subconjunto próprio de $\mathcal{L}\left(G, l^{p}\right)$.

Repetindo uma idéia utilizada por Lindenstrauss no artigo que estudamos no capítulo anterior, apresentaremos uma generalização do corolário anterior feita por Maria D.Acosta, Franscisco J.Aguirre e Rafael Payá ([A.A.P.1]).

\section{Teorema 4.11}

Considere a base de Schauder $\left(e_{i}\right)_{i \geq 1}$ associada ao espaço de Gowers $(G,\|\cdot\|) \in(Y,\|\cdot\|)$ qualquer espaço de Banach estritamente convexo.

Dado um operador $T \in \mathcal{N} \mathcal{A}(G, Y)$, existe $N_{T} \geq 1$ tal que $T\left(e_{i}\right)=0$ para todo $i \geq N_{T}$. Consequentemente, se houver um operador nâo compacto de $G$ em $Y$, podemos garantir que $Y$ não tem a propriedade $B$.

Prova: Admitindo sem perda de generalidade que $x \in G$ satisfaz $\|x\|=\|T x\|=\|T\|=1$, podemos pelo lema (4.7) garantir que $\left\|x \pm \delta \cdot e_{i}\right\|=1$ para $\delta>0$ e $i$ suficientemente grande (o símbolo " \pm " significando validade da expressão para ambos sinais). De

$$
\left\|T x \pm \delta \cdot T e_{i}\right\| \leq 1=\|T x\|
$$

e da convexidade estrita, a técnica presente na proposição (3.45) fornece o desejado. Repetindo-se o argumento apresentado na prova do corolário (4.10) tem-se também a consequência apresentada.

O resultado que apresentamos a seguir é devido à W.T.Gowers ([Gowers]).

\section{Corolário 4.12}

Todo espaço de Banach estritamente convexo $(X,\|\cdot\|)$ que contém uma cópia isomorfa à $l^{p} \quad(1<p<\infty)$ não admite a propriedade $B$.

Prova: De fato, se $X$ contém um subespaço isomorfo ao $l^{p}$, a imersão

$$
T: G \rightarrow l^{p} \hookrightarrow X
$$

constitui um operador não compacto de $G$ no subespaço referido e o teorema anterior se aplica. 


\subsection{Bishop-Phelps para bilineares}

\section{Definição 4.13 (forma bilinear)}

Considere $X$ um espaço vetorial. Uma aplicaçảo $\psi: X \times X \rightarrow \mathbb{R}$ é dita uma forma bilinear (real) se é linear em cada coordenada.

\section{Observação 4.14}

Quando tivermos $X$ normado consideraremos a partir daqui a norma no espaço-produto $X \times X$ dada pela aplicação

$$
\|(x, y)\|=\max \{\|x\|,\|y\|\} \quad, \quad \forall(x, y) \in X^{\prime} \times X^{\prime} .
$$

\section{Proposição 4.15}

Sejam um espaço normado $(X,\|\cdot\|)$ e $\psi: X \times X \rightarrow \mathbb{R}$ uma forma bilinear. Sáo equivalentes:

a) భ'é contínua.

b) $\psi$ é contínua na origem.

c) Existe uma constante real $M>0$ tal que $|\psi(x, y)| \leq M \cdot\|x\| \cdot\|y\|$, para qualquer $(x, y) \in X \times X$.

Prova: $a) \Rightarrow b$ ) : Imediato.

$b) \Rightarrow c)$ : Da continuidade de $\psi$ na origem temos, para $\varepsilon=1$, a existência de $0<\delta<1$ tal que

$$
\|\psi(x, y)\| \leq 1 \text { para qualquer }(x, y) \in X \times X,\|(x, y)\| \leq \delta .
$$

Caso $x=0$ ou $y=0$ a desigualdade é trivialmente válida para qualquer $M>0$. Do contrário, sejam $x_{p}=(\delta \cdot x) /\|x\|$ e $y_{p}=(\delta \cdot y) /\|y\| ;$ da bilinearidade da $\psi$ e de $\left\|\left(x_{p}, y_{p}\right)\right\|=\delta$ segue que

$$
\left|\psi\left(x_{p}, y_{p}\right)\right|=\delta^{2} \cdot \frac{|\psi(x, y)|}{\|x\| \cdot\|y\|} \leq 1
$$

e portanto $M=1 / \delta^{2}$ satisfaz o desejado. 
c) $\Rightarrow a)$ : Fixado qualquer ponto $z_{0}=\left(x_{0}, y_{0}\right) \in X \times X$. como

$$
\psi(x, y)-\psi\left(x_{0}, y_{0}\right)=\psi\left(x-x_{0}, y\right)+\psi\left(x_{0}, y-y_{0}\right)
$$

segue que, para $M>0$ da hipótese, teremos

$$
\begin{aligned}
\left|\psi(x, y)-\psi\left(x_{0}, y_{0}\right)\right| & \leq\left|\psi\left(x-x_{0}, y\right)\right|+\left|\psi\left(x_{0}, y-y_{0}\right)\right| \leq \\
& \leq M\left(\left\|x-x_{0}\right\| \cdot\|y\|+\left\|x_{0}\right\| \cdot\left\|y-y_{0}\right\|\right)
\end{aligned}
$$

Tomando $(x, y) \in X^{\prime} \times X^{\prime}$ tal que $\left\|(x, y)-\left(x_{0}, y_{0}\right)\right\| \leq 1$ temos facilmente

$\left|\psi(x, y)-\psi\left(x_{0}, y_{0}\right)\right| \leq M\left(\|y\|+\left\|x_{0}\right\|\right) \leq M\left(1+\left\|y_{0}\right\|+\left\|x_{0}\right\|\right) \leq M(1+2 c)$

onde $c=\max \left\{\left\|x_{0}\right\|,\left\|y_{0}\right\|\right\}$.

Dado $\varepsilon>0$, para $\delta=\min \left\{1, \frac{\varepsilon}{M(1+2 c)}\right\}>0$ segue, a partir da expressão (4.1), que

$$
\begin{aligned}
\left|\psi(x, y)-\psi\left(x_{0}, y_{0}\right)\right| & \leq M\left(\frac{\varepsilon}{M(1+2 c)}\|y\|+\left\|x_{0}\right\| \frac{\varepsilon}{M(1+2 c)}\right) \leq \\
& \leq M\left(\frac{\varepsilon}{M(1+2 c)}(1+c)+c \frac{\varepsilon}{M(1+2 c)}\right)=\varepsilon
\end{aligned}
$$

para todo $(x, y) \in X \times X,\left\|(x, y)-\left(x_{0}, y_{0}\right)\right\| \leq \delta$ (como desejado).

\section{Definição 4.16 (norma de bilinear)}

Dado um espaço normado $(X,\|\cdot\|)$ e uma forma bilinear contínua $\psi: X \times X \rightarrow \mathbb{R}$, definimos a norma de $\psi$ como sendo o número real

$$
\|\psi\|=\inf \{M>0:|\psi(x, y)| \leq M \cdot\|x\| \cdot\|y\|, \forall(x, y) \in \mathrm{X} \times X\} .
$$

\section{Observação 4.17}

Omitiremos a (simples) prova de que

$$
\|\psi\|=\sup _{(x, y) \in \mathcal{B}_{X}(\|\cdot\|) \times \mathcal{B}_{X}(\|\cdot\|)}|\psi(x, y)|,
$$

fato este que será usado a partir da proposição (4.20). 


\section{Definição 4.18 (bilinear atingir norma)}

Fixado um espaço de Banach $(X,\|\cdot\|)$, considere Bill $\left(X^{\prime}\right)$ como sendo o espaço constituido pelas formas bilineares contínuas em $X \times X$ a valores reais.

Diremos que $\longleftrightarrow \in \mathcal{B}$ ill $\left(X^{\prime}\right)$ atinge sua norma se existirem dois pontos $x_{0}, y_{0}$ em $\mathcal{B}_{X}(\|\cdot\|)$ tais que $\left|\psi \cdot\left(x_{0}, y_{0}\right)\right|=\|\psi\|$ : denotaremos por $\mathcal{B i l}_{N_{A}}\left(X^{\prime}\right)$ o subconjunto de Bill $\left(X^{X}\right)$ constituido pelas formas bilineares contínuas que têm tal propriedade.

\section{Exemplo 4.19}

Seja o espaço das funções $\mathcal{C}([0,1])=\{f:[0,1] \rightarrow \mathbb{R}, f$ contínua $\}$ munido da sua norma usual dada por

$$
\|f\|=\max _{t \in[0,1]}|f(t)| .
$$

Consideremos a aplicação $\psi: \mathcal{C}([0,1]) \times \mathcal{C}([0,1]) \rightarrow \mathbb{R}$ dada por

$$
\psi(x, y)=\int_{0}^{1} K(t) \cdot x(t) \cdot y(t) d t
$$

onde $K:[0,1] \rightarrow \mathbb{R}$ é qualquer aplicação contínua e tal que $K(t) \geq 0, \forall t \in[0,1]$.

Note que a bilinearidade da $\psi$ é imediata. Mostremos que ela é contínua e que atinge sua norma. Valendo-nos das propriedades associadas à integral temos

$$
\begin{aligned}
|\psi(x, y)| & =\left|\int_{0}^{1} K^{\prime}(t) \cdot x(t) \cdot y(t) d t\right| \leq \int_{0}^{1}\left|K^{\prime}(t)\right| \cdot|x(t)| \cdot|y(t)| d t \leq \\
& \leq\left(\max _{t \in[0,1]}|x(t)|\right) \cdot\left(\max _{t \in[0,1]}|y(t)|\right) \cdot \int_{0}^{1} K^{\prime}(t) d t=M \cdot\|x\| \cdot\|y\|
\end{aligned}
$$

onde $M=\int_{0}^{1} L^{-}(t) d t<\infty$.

Pela proposição (4.15) segue que $\psi$ é contínua. Tomando $x_{0}, y_{0}$ em $\mathcal{C}([0,1])$ tais que $x_{0}(t)=y_{0}(t)=1$ para todo $t \in[0,1]$, temos de imediato que

$$
\left|\psi\left(x_{0}, y_{0}\right)\right|=M=\|\psi\|
$$


donde segue que $\xi$ atinge sua norma.

O resultado a seguir, obtido por Maria D.Acosta, Franscisco J.Aguirre e Rafael Payá ([A.A.P.2]), terá como corolário o (contra-)exemplo que dá nome ao referido artigo e que encerra esta dissertação.

\section{Proposição 4.20}

Considere a base de Schauder $\left(e_{i}\right)_{i \geq 1}$ associada ao espaço de Gowers $(G,\|\cdot\|)$. Se $\psi \in \mathcal{B i l}_{N A}(G)$, então $\psi\left(\epsilon_{m}, \epsilon_{n}\right)=0$ para quaisquer índices $m, n$ suficientemente grandes.

Prova: Considere qualquer $\left(x_{0}, y_{0}\right) \in \mathcal{S}_{G}(\|\cdot\|) \times \mathcal{S}_{G}(\|\cdot\|)$ tal que

$$
\left|\psi\left(x_{0}, y_{0}\right)\right|=\|\psi\| \geq|\psi(x, y)|, \forall(x, y) \in \mathcal{S}_{G}(\|\cdot\|) \times \mathcal{S}_{G}(\|\cdot\|) .
$$

Pelo lema (4.7) sabemos existirem $\delta>0$ e $N_{0} \geq 1$ tais que (novamente entendendo o símbolo " \pm " como validade da expressão para ambos sinais)

$$
\left\|x_{0} \pm \delta \cdot e_{m}\right\|=\left\|y_{0} \pm \delta \cdot e_{n}\right\|=1, \forall m, n \geq N_{0}
$$

e, portanto, da bilinearidade de $\psi$ e também (4.2) podemos afirmar que $\left|\psi\left(x_{0}, y_{0}\right)\right| \geq\left|\psi\left(x_{0} \pm \delta \cdot e_{m}, y_{0}\right)\right|=\left|\psi\left(x_{0}, y_{0}\right) \pm \delta \cdot \psi\left(e_{m}, y_{0}\right)\right|, \forall m \geq N_{0}$.

Repetiremos aqui a técnica que explora a convexiclade estrita, neste caso no espaço $(\mathbb{R},|\cdot|)$. Para $m \geq N_{0}$ teremos

$2\left|\psi\left(x_{0}, y_{0}\right)\right| \leq\left|\psi\left(x_{0}, y_{0}\right)-\delta \cdot \psi\left(\epsilon_{m}, y_{0}\right)\right|+\left|\delta \cdot \psi\left(e_{m}, y_{0}\right)+\psi\left(x_{0} \cdot y_{0}\right)\right||\leq 2| \psi\left(x_{0}, y_{0}\right) \mid$, a última desigualdade obtida a partir de (4.3).

Uma vez que tenhamos $\left|\psi\left(x_{0}, y_{0}\right) \pm \delta \cdot \psi\left(e_{m}, y_{0}\right)\right|=2\left|\imath \cdot\left(x_{0} ; y_{0}\right)\right|$, segue (de $\delta>0$ ) que $\psi\left(e_{m}, y_{0}\right)=0$ para todo $n \geq N_{0}$ (procedendo-se como na proposição (3.45)).

Usando agora $\left\|y_{0} \pm \delta \cdot e_{n}\right\|=1 \quad\left(n \geq N_{0}\right)$, obtem-se da mesma forma $\psi\left(x_{0}, e_{n}\right)=0$, donde concluimos que

$$
\left|\psi\left(x_{0}, y_{0}\right)\right| \geq\left|\psi\left(x_{0} \pm \delta \cdot e_{m}, y_{0} \pm \delta \cdot e_{n}\right)\right|=\left|\psi\left(x_{0}, y_{0}\right) \pm \delta^{2} \cdot \psi\left(e_{m}, e_{n}\right)\right|
$$


desde que $m, n \geq N_{0}$.

Novamente a técnica presente na proposiçào (3.45) nos permite concluir que $\psi\left(e_{m}, e_{n}\right)=0$ para quaisquer $m, n \geq N_{0}$, como desejado.

\section{Corolário 4.21}

Seja $(G,\|\cdot\|)$ o espaço de Giowers. O conjunto $\mathcal{B i l}_{N A}(G)$ não é denso no espaço Bill $(G)$.

Prova: Levando-se em conta a imersão de $G$ no espaço $l^{p}$, podemos definir em $G \times G$ uma forma bilinear contínua $\psi$ dada por

$$
\psi(x, y)=\sum_{i \geq 1} x_{i} \cdot y_{i},
$$

com $x=\left(x_{i}\right)_{i \geq 1}$ e $y=\left(y_{i}\right)_{i \geq 1}$ vistos como elementos de $l^{p} \quad(1<p<\infty)$.

Tomando-se a base de Schauder $\left(e_{n}\right)_{n \geq 1}$ associada ao espaço $(G,\|\cdot\|)$, como

$$
\psi\left(e_{n}, e_{n}\right)=\sum_{i \geq 1} \epsilon_{n, i} \cdot \epsilon_{n, i}=\epsilon_{n, n} \cdot \epsilon_{n, n}=1, \forall n \geq 1
$$

temos, pela proposição anterior, que fixado qualquer $\varphi \in \mathcal{B}_{i l_{N A}}(G)$, existe $N_{0} \geq 1$ tal que $\varphi\left(e_{n}, e_{n}\right)=0$ para todo $n \geq N_{0}$ e, portanto,

$$
\|\psi-\varphi\| \geq\left\|(\psi-\varphi)\left(\epsilon_{n}, e_{n}\right)\right\|=1, \forall n \geq N_{0} .
$$

Assim sendo, $\psi$ nào pode ser aproximado por aplicaçòes bilineares contínuas que atingem suas respectivas normas.

"We must know. We will know."

(David Hilbert) 


\section{Referências Bibliográficas}

[A.A.P.1] M.D. Acosta, F. Aguirre and R. Payá, A Space by $W$. Gowers and New Results on Norm and Numerical Radius Attaining Operators, Acta Univ. Carol.-Math. et Phys. 33 (1992), 5-14

[A.A.P.2] M.D. Acosta, F. Aguirre and R. Payá, There is no bilinear BishopPhelps theorem, Israel J. Math. 93 (1996), 221-227

[A.F.W.] R.Aron, C.Finet \& E.Werner, Some remarks on norm-attaining n-linear forms, Lect. Notes in Pure and Appl. Math. 172 (1995), 19-28

[Bourgain] J.Bourgain, On dentability and the Bishop-Phelps property, Israel J. of Math., 284 (1977), 265-271

[B.P.] E. Bishop \& R.R.Phelps, A proof that every Banach space is subreflexive, Bull. Amer. Math. Soc. 67 (1961), 97-98

[Berberian] Berberian S.K, Lectures in Functional Analysis and Operator Theory, Springer-Verlag (1973)

[Brezis] Brezis H.. Analyse Fonctionnelle, thèorie et applications (2 tirage), Masson (198i)

[BrwnPrcy] Brown A. \& Pearcy C., An Introduction to Analysis, SpringerVerlag (1991)

[Day] Day M., Normed Linear Spaces, Academic Press (1962)

[Diestel] Diestel J., Geometry of Banach Spaces, Lect. Notes in Math. 485, Springer-Verlag

[Diestel2] Diestel J.. Sequences and series in Banach Spacts. Springer-Verlag 92 (1984) 
[Dunf.Schw.] Dunford \& Schwartz, Linear Operators - Part I, Interscience Publishers, New York (1967)

[DvlGdfr] Deville R. \& Godefroy G., Smoothness and Renormings in Banach Spaces, Longman Scientific \& Technical (1993)

[Edwards] Edwards R.E., Functional Analysis, Dover (1995)

[Elon] Lima, E.L., Curso de análise, volٌ ( $3^{\underline{a}}$ edição), Projeto Euclides (1989)

[Garling] Garling D.J.H., On symmetric sequence spaces, Proc.London Math.Soc. 16 (1966), 85-106

[Gowers] W.T. Gowers, Symmetric block basts of sequences with large average growth, Israel J. Math. 69 (1990), 129-151

[Holmes] Holmes R., Geometric Functional Analysis and its Applications, Springer-Verlag (1975)

[Istratescu] Istratescu, V.I., Strict convexity and complex strict convexity, Lect. Notes in Pure and Appl. Math. 89, Marcel Dekker (1984)

[James] James, R.C., Bases and reflexivity of Banach spaces, Bull. Amer. Math. Soc. 56 (1950), 58

[James2] James, R.C., Characterizations of reflexivity, Studia Math. 23 (1963), 205-216, MR 30/431

[Kadec] Kadec M.I., On spaces which are isomorphic to locally uniformly convex spaces, Izv. Vyssh. Ucheb. Zaved. (Mathematics) 6 (1959), 51-57

[Klee] Klee V., Some characterizations of reflexivity, Rev. Ci. (Lima) 52 (1950), 15-23

[Köthe] Köthe, G., Topological Vector Spaces I, Springer-Verlag 159 (1969)

[KtrAklv] Kantorovich, L.V.\& Akilov, G.P., Functional Analysis (2 edition), Pergamon Press (19S2)

[Larsen] Larsen, R., Functional Analysis - an introduction, Marcel Dekker (1973) 
[Lindenst] J. Lindenstrauss, On operators which attain their norm, Israel J. Math. 1 (1963), 139-148

[Lind.Tzaf.] Lindenstrauss J. \& Tzafriri L., Classical Banach Spaces I and II, Springer-Verlag (1996)

[Maddox] Maddox I.J., Elements of Functional Analysis (2 edition), Cambridge University Press (1988)

[Partington] J.R.Partington, Norm attaining operators, Israel J. of Math. 43 3 (1982), 273-276

[S.P.] Sevilla M.J \& R.Payá, Norm attaining multilinear forms and polynomials on preduals of Lorentz sequence spaces, Studia Math. 127 (2) (1998)

[Stegall] Charles Stegall, Optimization and differentiation in Banach spaces, Lin. Alg. Appl. 84 (1986), 191-211

[Zizler] V. Zizler, On some extremal problems in Banach spaces, Math. Scand. 32 (1973), 214-224. 